HIGH RESOLUTION TOMOGRAPHIC IMAGING OF THE ALVEOLAR REGION OF THE MAMMALIAN LUNG

A close look deep into the lung

Graduate School for Cellular and Biomedical Sciences

University of Bern

Ph.D. Thesis

Submitted by

DAVID HABERTHÜR

from Metzerlen-Mariastein, SO

\author{
Thesis Advisor \\ Prof. Dr. Johannes C. Schittny \\ Institute of Anatomy \\ Faculty of Medicine \\ University of Bern
}

May 2010 

HIGH RESOLUTION TOMOGRAPHIC IMAGING OF THE ALVEOLAR REGION OF THE MAMMALIAN LUNG

A close look deep into the lung

Graduate School for Cellular and Biomedical Sciences

University of Bern

Ph.D. Thesis

Submitted by

DAVID HABERTHÜR

from Metzerlen-Mariastein, SO

Thesis Advisor

Prof. Dr. Johannes C. Schittny

Institute of Anatomy

Faculty of Medicine

University of Bern

May 2010

\section{(9) $(1) \Theta$}

This work is licensed under a Creative Commons

Attribution-NonCommercial-NoDerivs 2.5 Switzerland license. To see the license go to creativecommons.org/licenses/by-nc-nd/2.5 or write to Creative Commons, 171 Second Street, Suite 300, San Francisco, California 94105, USA. 


\section{Copyright Notice:}

This document is licensed under the Creative Commons AttributionNon-Commercial-No derivative works 2.5 Switzerland license: creativecommons.org/licenses/by-nc-nd/2.5.

You are free to copy, distribute, display, and perform of this work under the following conditions:

- You must give the original author credit.

- You may not use this work for commercial purposes.

- You may not alter, transform, or build upon this work.

- Chapters 5, 6 and 7 of this work are publications authored for several journals. Chapter 5 was published as an open access proceeding in the Journal of Physics: Conference Series and is freely distributable. Chapters 6 and 7 were published in the Journal of Applied Physiology and the Journal of Synchrotron Radiation, respectively. Both these chapters are covered by copyright from their Journals.

For any reuse or distribution, you must take clear to others the license terms of this work. Any of these conditions can be waived if you get permission from the copyright holder. Nothing in this license impairs or restricts the author's moral rights according to Swiss law.

The detailed license agreement can be found at: creativecommons.org/ licenses/by-nc-nd/2.5/ch/legalcode.de

David Haberthür

High resolution tomographic imaging of the alveolar region of the mammalian lung A close look deep into the lung

SVN-Revision 67

SUPERVISORS:

Prof. Dr. Johannes C. Schittny

Dr. Mauricio Reyes

Prof. Dr. Martin Frenz

LOCATION:

Bern, Switzerland

TIME FRAME:

September 2006-May 2010 
Accepted by the Faculty of Medicine, the Faculty of Science and the Vetsuisse Faculty of the University of Bern at the request of the Graduate School for Cellular and Biomedical Sciences.

Bern,

Dean of the Faculty of Medicine

Bern,

Dean of the Faculty of Science

Bern,

Dean of the Vetsuisse Faculty Bern 

Dedicated to everyone who shared a path on my journey to this point. 

Studying lung development in all its characteristics, especially analyzing the structural parameters of the terminal airways needs a fully three-dimensional imaging modality, since not all structural parameters can be assessed on classic histological sections.

Using synchrotron radiation based tomographic microscopy threedimensional volumetric information of arbitrary samples can be obtained. The distinct advantages offered by tomographic microscopy make it an extremely well suited imaging method for the minute analysis of lung samples in three dimensions. Tomographic microscopy permits to study the samples in a non-destructive way and allows the acquisition of tomographic datasets with ultra high resolution in the micrometer scale in a short time, usually within a few minutes.

This work presents several methods for the analysis of the terminal airway using high resolution tomographic datasets. Using a combination of this imaging modality with transmission electron microscopy data we localized the deposition sites of sub-micrometer sized particles in the terminal airways and studied their properties.

Three-dimensional tomographic reconstructions were used for the analysis of structural parameters of the terminal airways. The achieved results confirmed the usability of tomographic data for confirming structural parameters obtained from classic histological slices, which highlights the advantage of the non-destructive scanning method.

The third method presented in this work makes it possible to record tomographic dataset of large sample volumes with ultra high resolution. Using such datasets, we analyzed structural changes of the terminal airways of the mammalian lung during postnatal lung development. The presented method achieves a breakthrough for tomographic imaging, since generally a large field of view had to be traded for a high resolution, which is no longer necessary with the application of the so-called wide field scanning.

In the context of this work large means with a volume of several cubic millimeters. 



\section{ZUSAMMENFASSUNG}

Um die Lungenentwicklung und die strukturellen Parameter der terminalen Luftwege detailliert analysieren zu können, muss ein dreidimensionales Abbildungsverfahren verwendet werden, denn auf klassischen histologischen Schnitten des Lungengewebes können nicht alle strukturellen Parameter erfasst werden.

Mit tomographischer Mikroskopie basierend auf Synchrotronstrahlung können solche dreidimensionalen volumetrische Informationen von praktisch beliebigen Proben gewonnen werden. Die tomographische Mikroskopie bietet verschiedene Vorzüge, welche sie zu einer bestens geeigneten Methode machen, um Lungenproben minuziös in drei Dimensionen zu untersuchen; namentlich können die Proben zerstörungsfrei untersucht werden sowie bieten die resultierenden Daten höchste Auflösungen im Mikrometerbereich und können in kürzester Zeit, normalerweise innerhalb weniger Minuten aufgenommen werden.

Die vorliegende Arbeit präsentiert mehrere Methoden, um die terminalen Luftwege der Lunge mittels höchstaufgelösten tomographischen Daten zu untersuchen. Durch eine Kombination dieser Daten mit Elektronenmikroskopiebildern konnten wir die genauen Orte der Ablagerung von Partikeln kleiner als ein Mikrometer in den terminalen Luftwegen lokalisieren und die genauen Eigenschaften der Partikel untersuchen.

Weiter haben wir dreidimensionale Rekonstruktionen von tomographischen Daten benutzt, um die struturellen Parameter der terminalen Luftwege zu untersuchen. Der Vergleich der erlangten Resultate mit Resultaten, welche aufgrund von klassischen Histologieschnitten erhoben wurden zeigt, dass diese strukturellen Parameter genausogut auf tomographischen Daten erhoben werden können, ohne die Genauigkeit der Resultate einzuschränken. Dies hebt den Vorteil der zerstörungsfreien tomographischen Abbildungsmethode hervor.

Die dritte Methode, welche in dieser Arbeit präsentiert wird, ermöglicht es, tomographische Datensätze von grossen Lungenvolumina in höchster Auflösung aufzunehmen. So aufgenommene Datensätze ermöglichen es, Strukturänderungen der terminalen Luftwege in der Säugetierlunge während der postnatalen Lungenentwicklung zu untersuchen. Das vorgestellte Verfahren stellt einen Durchbruch in der tomographischen Mikroskopie dar, denn bis jetzt ging eine Vergrösserung der abgebildeten Volumina immer mit einer Verkleinerung der Auflösung der erzielten Daten einher. Dieser Nachteil konnte mit der neu entwickelten, sogenannten wide field scanning Methode überwunden werden. 

You'll never walk alone

- Johnny Cash [1]

\section{ACKNOWLEDGMENTS}

I thank Prof. Dr. Johannes C. Schittny for giving me the opportunity to carry out this work at the Institute of Anatomy at the University of Bern. Sharing his vast knowledge, Johannes introduced me into the delicate details of lung morphology, physiology and development and also provided multiple enjoyable moments away from the lab routine.

Dr. Miguel Gonzàlez helped me to get on track with various image processing intricacies and was-after changing job from the Institute for Surgical Technology \& Biomechanics (ISTB) in Bern to Alma IT Systems in Spain - very well substituted by Dr. Mauricio Reyes from the ISTB, which watched over the second half of my time working for this thesis. Both Miguel and Mauricio deserve thanks for their guidance as co-referees of this work. Prof. Dr. Martin Frenz acted as my mentor for the Graduate School for Cellular and Biomedical Sciences of the University of Bern. Already during my Master thesis I had the pleasure to work under the guidance of Martin and I am thankful for his watching eye as a mentor.

Both Sébastien Barré and Lilian Salm shared the office with me during the last months at the Institute of Anatomy and contributed to the pleasant atmosphere in our group. Sébastien was a helpful discussion partner for all MATLAB problems and during countless hours trying to stay awake and alert during numerous beamtime shifts at TOMCAT. Lilian spent many kyphotic hours counting alveolar bridges with the STEPanizer and is responsible for the nice counting results shown in the discussion. Mohammed Ouanella was an expert help with the preparation of the samples and also supplied excellent food from his home country, Algeria. Thanks!

The whole staff at the Institute of Anatomy provided for a great time; special thanks go to Dr. Stefan Tschanz for unconditional help with all things IT and for putting up with my special wishes concerning the use of the computing infrastructure here at the institute. Chrigu Lehmanns steady hand was welcome for the lathe machining o.6 mm Epon samples before we switched to paraffin embedded "big" lung samples. He also always provided generous help with special mechanical tasks, be it work related or not, e. g. like a riveting a broken buckle back to my

She's a med student, she knows what kyphotic means... 
Note that both my sister and girlfriend share the same first name, but are different persons. Having said that, I thank all the important Ninas in my life for the help with proofreading this work. ski boots. Our secretary team and especially Therese Dudan always provided the first friendly smiles in the morning when I came in to pick up our mail.

Special thanks go to the whole team at the TOMCAT beamline at the Swiss Light Source of the Paul Scherrer Institut in Villigen; without them, none of the awesome images in this thesis would exist. Prof. Dr. Marco F. M. Stampanoni, head of the TOMCAT beamline at the Swiss Light Source and assistant Professor for X-ray Microscopy at the Institute for Biomedical Engineering of the ETH in Zürich provided a pleasurable work environment and was an invaluable resource on all things tomography and x-rays, provided well founded critique for my work and helped me to brush up my Italian skills both written and spoken. Dr. Christoph Hintermüller was not only a great guidance during my Master thesis for the postgraduate course in Medical Physics but provided expert support at the beamline and countless interesting discussions, both scientific and completely non-scientific. Xris' insight into scripting, command line tricks, tomography and image processing was a great help. Without the work of Dr. Federica Marone on the tomographic reconstruction algorithms and implementation of those algorithms at TOMCAT the wide-field-scan tomographic reconstructions in this work would not exist.

The University sports Berne, the Velokurier Bern and Fabio Breil with the eccentric bike provided me with some athletic balance to the time spent at the desk in front of my monitors.

My friends Pesche, Bruni, Wöufu, Dr. Sigi and Nicola as well as Mara and Barbara provided (sometimes much needed) diversion from the academic turf and shared loads of lunch times. Dan and Tobi as my former flat mates also helped to wrap my brain around different stuff than academics. Thanks!

Without the constant support from my family I would not be where I am today. Mami, Papi and Nina, I'll always be grateful to all of you.

Nina, you are the best supporter, discussion partner, sport teammate, friend, flatmate, reviewer, gardener, cook and girlfriend one could wish for. Your love makes me happy. I $\odot$ you! 
I INTRODUCTION 1

1 INTRODUCTION 3

1.1 High resolution imaging 3

1.2 The mammalian lung 4

1.3 Main goal of the Thesis 5

1.4 Outline of the Thesis 7

2 The mammalian Lung 9

2.1 Lung Development 9

2.2 The functional units of the lung 11

2.3 The need for Tomography 11

3 COMPUTED TOMOgRAPHY 15

3.1 History 15

3.2 Interaction of radiation with Matter 16

3.2.1 Absorption 17

3.2.2 Scattering 17

3.2.3 Attenuation 18

3.3 From Projections to Slices: Computed tomography 19

3.4 Synchrotron Radiation 22

3.4.1 Bending Magnets, Undulators and Wigglers 22

3.5 The Swiss Light Source 24

3.6 TOMCAT 24

II RESULTS 29

4 PUBLications 31

4.1 chapter 5 - Multimodal imaging 31

4.2 chapter $6-\mathrm{FE} 3 \mathrm{D}$ reconstruction of the acinus 32

4.3 chapter 7 - Expansion of the field of view 32

5 MUltimodal imaging OF SUb-Micron PARTiCles 35

5.1 Abstract 35

5.2 Objective 36

5.3 Materials and Methods 36

5.4 Results 36

5.5 Discussion 36

5.6 Conclusions 38

5.7 Acknowledgments 39

6 FE 3D RECONSTRUCTION OF THE PULMONARY ACINUS 41

6.1 Abstract 41

6.2 Introduction 42

6.3 Materials and Methods 43

6.3.1 Animal Handling 44

6.3.2 SRXTM 44

6.3.3 Validation of Segmentation \& Image Processing 49

6.3.4 Visualization of the Reconstruction 49

6.4 Results 49

6.4.1 A Raw Image Stack and a Cubic Subregion 49

6.4.2 Segmentation 51

6.4.3 FE 3D reconstruction 51

6.4.4 Validation of Segmentation \& Image Processing 51

6.4.5 FE 3D Reconstruction of Air Space 53 
6.5 Discussion 54

6.5.1 Historical Perspective 54

6.5.2 SRXTM 56

6.5.3 Segmentation/Threshold 57

6.5 .4 2D vs. 3D 58

6.5.5 FE 3D Rendering 59

6.6 Acknowledgments 60

7 RADIATION DOSE OPTIMIZED EXPANSION OF THE FIELD

OF VIEW 61

7.1 Abstract 61

7.2 Introduction 62

7.3 Materials and Methods 63

7.3.1 Sample Preparation 63

7.3.2 Synchrotron radiation tomographic microscopy

$7 \cdot 3 \cdot 3$ Increasing the field of view 63

7.3.4 Quality guided protocols 66

7.3.5 Projection merging \& tomographic reconstruction 68

7.4 Results 69

7.4.1 Image Merging and Reconstruction 69

7.4.2 Performance of the scanned protocols 69

7.4.3 3D visualization of different protocols $\quad 72$

7.5 Discussion 75

7.6 Summary 78

7.7 Acknowledgments 79

III DISCUSSION \& OUTLOOK 81

8 Discussion 83

8.1 Multimodal Imaging 84

8.2 Finite element reconstruction of the terminal airways 85

8.3 WF-SRXTM 87

8.3.1 Skeletonization 88

8.3.2 Stereological analysis of the tomographic data 89

9 OUTLOOK 93

IV BACK MATTER 95

Curriculum Vitæ and selected Publications 97

Bibliography 101

Declaration of Originality 117

Colophon 119 
Figure 2.1 Lung development stages, classical paradigm 10

Figure 2.2 Lung development stages, revised paradigm $\quad$ II

Figure 2.3 Details of the human lung $\quad 12$

Figure 3.1 Interaction types 18

Figure 3.2 Total attenuation coefficient of Ce:YAG 19

Figure $3.3 \quad$ Correction of the projections with flat images 20

Figure $3.4 \quad$ Sinogram and Reconstruction 21

Figure 3.5 Bending magnet radiation 23

Figure 3.6 Undulator radiation 23

Figure 3.7 Wiggler radiation 24

Figure 3.8 Beamlines at the SLS 25

Figure 3.9 The TOMCAT beamline 27

Figure 5.1 3D visualization of gold particles in the lung 37

Figure 5.2 TEM images of gold particles 38

Figure 6.1 Workflow for 3D reconstruction of the acinus 43

Figure 6.2 SRXTM imaging setup 45

Figure 6.3 Object connectivity analysis 47

Figure 6.4 Grid-based hexahedral algorithm 50

Figure 6.5 Overview of workflow for ROI selection 52

Figure 6.6 Thresholding Influence 53

Figure 6.7 3D reconstruction of tissue 54

Figure 6.8 Tissue volume- and airspace surface area-density 55

Figure 6.9 3D reconstruction of air spaces 56

Figure 6.10 3D FE shell model of a partial acinus 58

Figure 7.1 Covering the field of view of different samples 64

Figure 7.2 Wide field scan setup 65

Figure 7.3 Setup for different field of views 67

Figure $7.4 \quad$ Workflow of a wide field scan 70

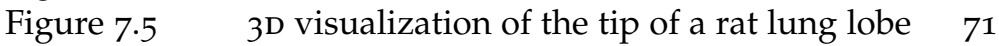

Figure 7.6 Plot normalized difference value 73

Figure 7.7 Comparison of three-dimensional visualizations

Figure 7.8 Location of the four ROIs in the sample 75

Figure 7.9 Histogram-Plots 76

Figure 8.1 $\quad$ SEM image of a rat lung sample $\quad 84$

Figure 8.2 Multimodal Imaging 86

Figure 8.3 2D skeletonization 88

Figure 8.4 3D Visualization of rat lung sample 89

Figure 8.5 Manhole cover process 90

Figure 8.6 Alveolar entrance rings per volume 92 
LIST OF TABLES

Table 7.1 Details of the 19 scanned protocols 68 
AMD

AVV

$\mathrm{BP}$

CAD

CCD

CFD

CT

CPU

DAT

DCMM

EEG

EPICS

EM

ETH

FE

FFT

GBHA

ISTB

IT

LM

LST

$\mu \mathrm{CT}$

MATLAB

MRI

OCA

Advanced Micro Devices, Inc.

I have no idea what an AVV is, but it's used in a Monty Python sketch...

Blood Pressure

Computer Aided Design

Charge-Coupled Device

Computational Fluid Dynamics

Computed Tomography

Central Processing Unit

Proprietary file format for FE analysis

Double Crystal Multilayer Monochromator

Electroencephalography: The electrical activity of the brain can be measured with an EEG.

Experimental Physics and Industrial Control System: Control system by the national laboratory of Argonne in the USA, used to control TOMCAT.

Electron Microscopy: An electron microscope produces an electronically magnified image of a specimen for detailed observation. The electron microscope uses an electron beam to illuminate the specimen and create a magnified image of it.

Eidgenössische Technische Hochschule

Finite Element

Fast Fourier Transform

Grid-Based Hexahedral Algorithm

Institute for Surgical Technology \& Biomechanics

Information technology

Light microscopy

Laplacian Smoothing Technique

micro-Computed Tomography

MATrix LABoratory: MATLAB is a numerical computing environment developed by The MathWorks, Inc.

Magnetic Resonance Imaging

Object Connectivity Analysis 


\begin{tabular}{|c|c|}
\hline OLN & Object Label Number \\
\hline PC & Personal Computer \\
\hline PSI & $\begin{array}{l}\text { Paul Scherrer Institut: The PSI is the largest research } \\
\text { centre for natural and engineering sciences in } \\
\text { Switzerland. }\end{array}$ \\
\hline RAM & Random-Access Memory \\
\hline ROI & Region of Interest \\
\hline SE & $\begin{array}{l}\text { Standard Error: Standard deviation of a sample } \\
\text { normalized by the square root of the sample size. }\end{array}$ \\
\hline TEM & $\begin{array}{l}\text { Transmission Electron Microscopy: TEM is an electron } \\
\text { microscopy technique whereby a beam of electrons is } \\
\text { transmitted through an very thin specimen. }\end{array}$ \\
\hline TOMCAT & $\begin{array}{l}\text { TOmographic Microscopy and Coherent rAdiology } \\
\text { experimenTs: TOMCAT is the beamline at the SLS where } \\
\text { all experiments of this thesis have been performed. }\end{array}$ \\
\hline SEM & $\begin{array}{l}\text { Scanning Electron Microscopy: SEM is an electron } \\
\text { microscopy technique that images the sample surface } \\
\text { by scanning it with a high-energy beam of electrons in } \\
\text { a raster scan pattern. }\end{array}$ \\
\hline SLS & $\begin{array}{l}\text { Swiss Light Source: The SLS at the PSI is a } \\
\text { third-generation synchrotron light source. }\end{array}$ \\
\hline SNR & Signal-to-Noise Ratio \\
\hline SR & Synchrotron Radiation \\
\hline SRCT & Synchrotron Radiation Computed Tomography \\
\hline SRXTM & $\begin{array}{l}\text { Synchrotron Radiation based X-ray Tomographic } \\
\text { Microscopy }\end{array}$ \\
\hline STL & $\begin{array}{l}\text { Surface Tessellation Language or Standard Tessellation } \\
\text { Language: A file format used as a Quasi-Standard of } \\
\text { many CAD-systems. }\end{array}$ \\
\hline SVN & $\begin{array}{l}\text { Subversion: An open source version control system } \\
\text { used for revision control of this work. }\end{array}$ \\
\hline VRML & Virtual Reality Modeling Language \\
\hline WF-SRXTM & $\begin{array}{l}\text { Wide Field Synchrotron Radiation Based X-ray } \\
\text { Tomographic Microscopy: The method to increase the } \\
\text { field of view of tomographic imaging stations } \\
\text { presented in chapter } 7 \text {. }\end{array}$ \\
\hline XML & $\begin{array}{l}\text { Extensible Markup Language: A set of rules for } \\
\text { electronic encoding of documents. }\end{array}$ \\
\hline YAG & Yttrium Aluminium Garnet \\
\hline
\end{tabular}


Part I

INTRODUCTION 

It's a magical world, Hobbes, ol' buddy... ... Let's go exploring!

- Calvin

Calvin and Hobbes, by Bill Watterson [2]

Seeing and observing the object of interest has always been a crucial part of medical and biological research. If not by the naked eye, the object has been studied with ever-increasing resolution. In biomedical research, state of the art imaging methods include light, x-ray and electron microscopy. Using advanced imaging methods like electron or x-ray microscopy, resolutions in the nanometer scale can be reached, which enables an extremely accurate rendition of the sample to be examined. Using three-dimensional methods like tomographic Electron Microscopy (EM) or especially Synchrotron Radiation based X-ray Tomographic Microscopy (SRXTM) samples can be studied with a resolution in the nanometer [3] or sub-micrometer [4] range.

\subsection{HIGH RESOLUTION IMAGING}

One of the main problems with light and electron microscopy is the destructive sample preparation. For both methods, the sample has to be cut into physical sections with a thickness between 50-300 nm, (EM, generally around $80 \mathrm{~nm}$ ) or 3-10 $\mu \mathrm{m}$ (LM). This process destroys the three-dimensional structure of the sample and makes it very time consuming to reconstruct the three-dimensional stack of physical sections to extract the structural information. Woodward and Maina [5] have shown that a three-dimensional reconstruction of parts of the gas exchanging region of the avian lung is feasible, but the process is both extremely time-consuming and needs very precise registration of the stack of sections which are cut from the sample.

An exception to this destructive sample preparation is offered by confocal microscopy [6]. In confocal microscopy, light originating from outside the focal plane is excluded using a spatial pinhole. This enables the analysis of a sample section which is thicker than the optical focal plane of the objective. By positioning the focal plane stepwise 
A voxel or volumetric pixel is the 3D analogy to a 2D pixel.

One exception being three newly discovered species of the animal phylum Loricifera which live inside permanently anoxic sediments [9].

An overview of the development and the relevant structures of the lung is given in chapter 2. through the volume of interest, the three-dimensional information of the sample can be obtained. This method obviously only works for samples showing a reasonably good penetration depth of the used light. The achievable volume of interest of confocal microscopy is thus limited and not sufficient for the analysis of the lung structure as presented in this work.

In contrast to this, tomographic imaging makes it possible to nondestructively study the three-dimensional structure of a wide variety of samples, even opaque ones. Two-dimensional projections of the sample can be taken fairly easily using a multitude of electromagnetic radiation of different wavelengths (e.g. $x$-ray, infrared and visible light). These two-dimensional images contain the partial three-dimensional information of the sample volume which has been transversed by the radiation used to record the projection. If several projection images are acquired from different directions through the sample, the full threedimensional information can be reconstructed using computed tomography [7]. Synchrotron Radiation based X-ray Tomographic Microscopy (SRXTM) is a method to provide this three-dimensional information of millimeter sized samples in high resolution with voxel sizes in the micrometer range [8]. SRXTM was used as an underlying tool to obtain this information for all the samples presented in this thesis. The theory and concepts behind the tomographic reconstruction are explained in depth in chapter 3 .

\subsection{THE MAMMALIAN LUNG}

Oxygen is an essential element for nearly every living multicellular organism. The mammalian lung is the organ that transports this element into the body and provides the structure for the gas-exchange of oxygen and carbon dioxide between the inhaled air in the alveolar space and the blood flowing in the capillaries in the lung tissue.

In the human body a tissue volume of approximately half a litre separates roughly the same amount of blood from an air volume of approximately 5 litres [10], the lung is thus mostly built "out of thin air". The actual tissue portion of the lung is stretched up in the chest cavity and fills it with a delicate structure. The larger, purely conducting airways are built of multi-layer epithelial tissue and the finest parts, the alveolar septa are built of two thin cytoplasmic lamellæ of the endothelial and type 1 epithelial cells separated by a single basement membrane; the average thickness of this tissue barrier is only about $1.6 \mu \mathrm{m}$ in the human lung [10].

The structure of the lung and the characteristics of the airways are vital for the function as an air conducting organ. Studying this structure makes it possible to gain important insights into both the development $[11,12]$ and function of the lung [13]. Three-dimensional imaging methods like Computed Tomography (CT) can give an insight into the structural relationships inside the lung, but cannot visualize the gas-exchange region of the lung through their limitation in resolution.

Tomographic imaging methods with higher resolutions like microComputed Tomography $(\mu \mathrm{CT})$ [14] and SRXTM [15-19] make it possible to extend the analysis deep into the lung, down to the gas-exchange region.

Certain structural parameters of the lung and especially of the terminal airway region as shown in Figure 1.1 cannot be assessed using 
classical morphological methods based on histological sections of the lung tissue, since this slicing essentially destroys the three-dimensional structure of the lung. For example, extracting a three-dimensional view of multiple functional units of the lung, the so-called acini and their interrelation is not possible only with the information from light or electron microscopy sections. But a study of their relations and structural changes is needed to characterize the changes the acini and lung structure undergo over the time-course of lung development. Using an imaging method like SRXTM that provides a full, unhindered and non-destructive high resolution view inside the lung makes it possible to reach this goal.

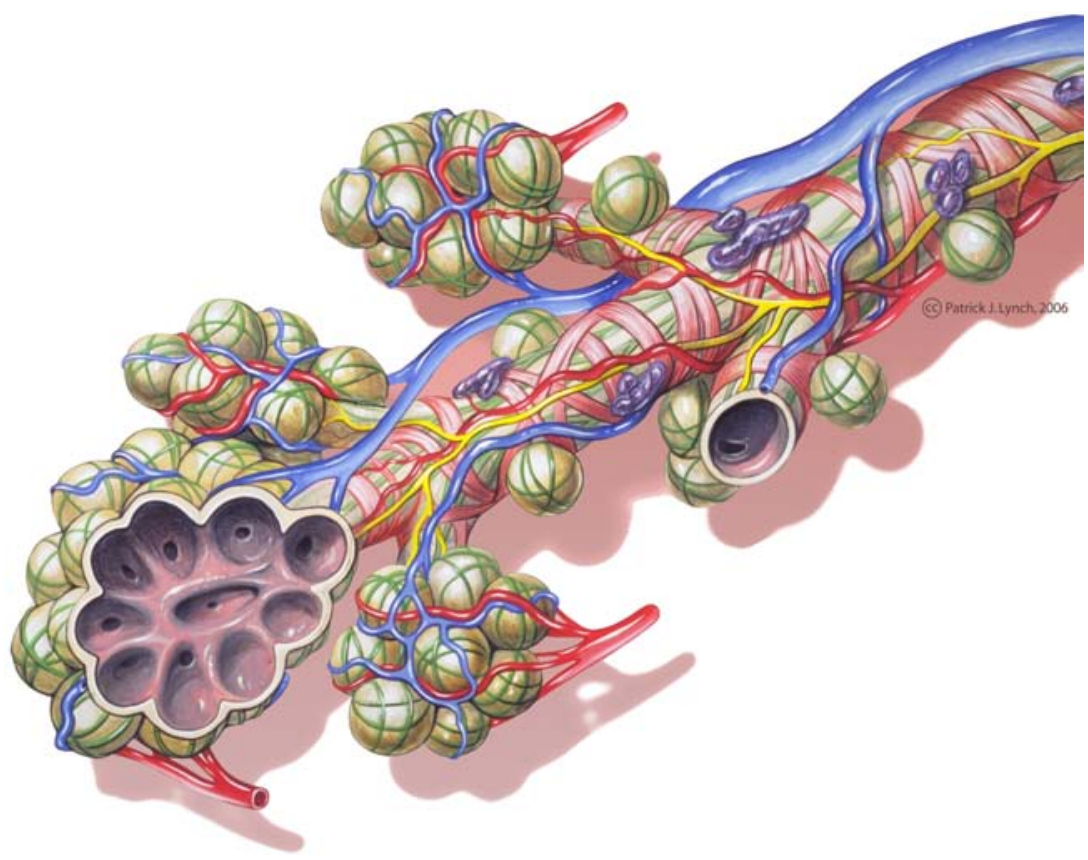

Figure 1.1: Bronchial anatomy detail of alveoli and lung circulation. Adapted from [20].

\subsection{MAIN GOAL OF THE THESIS}

From the beginning of my thesis at the Institute of Anatomy in September 2006 nearly everything I have worked on revolved around nondestructive tomographic imaging and detection of the finest structures in the mammalian lung, the so-called alveoli.

The main focus of the work in our group lies on the study of the postnatal lung development, in particular the changes in the airway structure [21]. As Weibel [10] described, the functional capacity of the lung as a gas exchange organ is determined by the fractal geometry, structure and peculiarities of the airways, almost opposite to the industrial design mantra Form follows function. Structural changes in the terminal airways like changes in alveolar number, increase in volume and changes in airway diameter [21] can be best studied using a fully three-dimensional imaging method like ultra high resolution tomographic imaging. In close collaboration with the team of the beamline for TOmographic Microscopy and Coherent rAdiology experimenTs (TOMCAT) at the Swiss Light Source (SLS) we recorded a
With formidable effort, Woodward and Maina [5] managed to visualize components of the gas-exchange region of a duck. 
large number of tomographic scans of rat lung samples over the postnatal lung development up until postnatal day 60 .

Modeling the structural parameters of the terminal airways using SRXTM data was one of the first goals achieved in this thesis. In a collaborative study I provided the three-dimensional reconstructions of multiple terminal airway regions inside several rat lung samples. Matching the structural parameters of the tomographic reconstructions to the same parameters previously assessed on two-dimensional histological sections from well characterized samples allowed an accurate reconstruction of the terminal airways in three dimensions. To our knowledge, the reconstructions of the alveolar and terminal airway region of rat lungs shown in chapter 6 are the first ever published in a peer-reviewed publication. In a subsequent collaborative study, this data has been used for Computational Fluid Dynamics (CFD) simulations of the airflow characteristics inside the alveoli, greatly increasing the fidelity of previous models of airflow deep in the lung [13].

Another question approached during my thesis was focused on the interaction of particles in the lung parenchyma. To fully understand the influence of the terminal airway structure on the deposition sites of inhaled particles a three-dimensional visualization method based on tomographic data is needed, two-dimensional data is not sufficient for the full understanding of the interrelations of the terminal airways. Tomographic data is a perfectly suited tool for the three-dimensional structure of the terminal airways and localization of inhaled particles, but an exact analysis of the properties of such inhaled particles is not possible using SRXTM datasets. Since the particle size is in the order of the voxel size or resolution of the tomographic data around $700 \mathrm{~nm}$, additional imaging methods like EM are needed for a full analysis of the submicron particles in relation to the lung tissue. Using a multimodal approach combining three-dimensional high resolution SRXTM data and two-dimensional ultra high resolution two-dimensional EM data the interaction of particles deep in the lung and their exact localization in the airway tree has been studied; these results are presented in chapter 5 .

During the first experiments which focused on the changes of the structural parameters of the terminal airway over the course of the lung development the correct positioning of the lung samples in the field of view at the TOMСAт beamline often relied on luck. This was because it is not possible to a judge or see the extent of single acini or connected airway segments inside a region of interest of $1.5 \times 1.5 \times 1.5 \mathrm{~mm}$ which corresponds to the field of view available at TOMCAT prior to tomographic imaging.

More precisely,

- the acinus is bigger than the field of view at the magnification needed to resolve the finest structures in the lung, the tissue septa between the alveoli in the terminal airways and

- the acinus seems to be growing over time during lung development.

One goal of this thesis was to solve the problem stated in the first point. I developed a method to increase the field of view of tomographic endstations-especially TOMCAT—while keeping the resolution of the resulting tomographic images on the level needed for the analysis of the finest structures in the lung. This goal was reached with 
the implementation of the so-called Wide Field Synchrotron Radiation Based X-ray Tomographic Microscopy (WF-SRXTM) scanning method at TOMCAT and has subsequently been published for the application at other tomographic endstations.

The second point mentioned above is the scope of ongoing research; analyzing the structure and size of the acini using a combination of segmentation and skeletonization of acini inside WF-SRXTM datasets already led to first promising results. The outlook in chapter 9 presents an overview of the achieved result and planned work.

\subsection{OUTLINE OF THE THESIS}

This thesis is structured into the following parts:

Part i contains this introduction as a first chapter. chapter 2 gives a short overview over the lung development. chapter 3 explains the most important concepts in computed tomography and gives a short description of the TOMCAT beamline, where all high resolution tomography experiments of this work have been performed.

Part ii contains publications written during the time of my Ph.D. thesis at the Institute of Anatomy, which present the most exiting results achieved during my time at the Institute of Anatomy.

chapter 5 consists of a proceeding written for the $9^{\text {th }}$ International Conference on X-Ray Microscopy in Zürich, Switzerland, from the $21^{\text {st }}$ to the $25^{\text {th }}$ July 2008 . This proceeding describes a method to precisely localize sub-micron sized gold particles in rat lungs through a combination of high resolution Synchrotron Radiation based X-ray Tomographic Microscopy and Transmission Electron Microscopy and has been published in the Conference Series of the Journal of Physics.

chapter 6 consists of a paper published in the Journal of Applied Physiology in which a novel image processing method for accurate three-dimensional reconstruction of the gas exchange region of the lung is presented. This method is based on a finite element analysis of datasets obtained with high resolution Synchrotron Radiation based X-ray Tomographic Microscopy.

chapter 7 presents a method to increase the field of view of tomographic microscopy while keeping the resolution of the resulting data on the desired high level, something which is generally not possible. Additionally-through optimization of the acquisition protocol-the radiation dose inflicted on the sample can be greatly reduced. This paper is currently in press and will be published in the Journal of Synchrotron Radiation.

Part iii contains two chapters with a conclusive discussion of the obtained results and an outlook on further work.

Part iv finishes this thesis with a Curriculum Vitæ, the Bibliography and the necessary documents for the Graduate School for Cellular and Biomedical Sciences.

In image processing, the skeletonization of an arbitrary structure results in a thin version of said shape which is equidistant to the shape boundaries. The skeletonization process used in this thesis is described in chapter 8. 

If we're gonna move forward, this is the next logical step! - Kevin Bacon

as Sebastian in The Hollow Man [22]

The lung is, in relation to the surface one of the largest mammalian organs. It has the second biggest inner surface after the intestinal tract, which has a size of approximately $320 \mathrm{~m}^{2}$ [23], the skin being distant third with approximately $2 \mathrm{~m}^{2}$ [24]. Both organs are involved in the uptake of the most important, vital substances like oxygen, water and sugar. The function of the lung is essential for the transport of oxygen from the air we breathe into the blood and for the transport of carbon dioxide from biological processes out of the blood stream back into the atmosphere.

The lung can fulfill its function through its design, which is specifically optimized to provide a large gas exchange surface-in humans an area equivalent to about $3 / 4$ of a tennis court, $130 \mathrm{~m}^{2}$ [10]-where capillary blood efficiently gets in close contact to the air inside the lung structure. The large area maximizes the region where the oxygen uptake can take place and a very thin tissue barrier maximizes the diffusion of the oxygen into the blood stream.

\subsection{LUNG DEVELOPMENT}

During lung development all the internal pulmonary structures like the conducting airways, the network of blood vessels and the gas exchange area are formed.

Mammalian lung development can be divided into five overlapping stages $[11,27]$, which are shown in the gray region in Figure 2.1: Organogenesis starts with a outpouching of the foregut resulting in the appearance of the lung buds and formation of the major airways. Subsequently, the conducting airways and parts of the respiratory airways are formed by a successive cycle of branching and growth starting at the lung buds, which is called branching morphogenesis. Most of this process takes place during the pseudoglandular stage. In this stage the

Keep in mind that that the measured length of area depends on the scale of measurement, as first described by Richardson [25] and popularized by Mandelbrot [26]. 
functional respiratory lung unit, the so-called acinus is formed. An acinus is defined as the complex of alveolated airways distal of a last purely conducting airway, the terminal bronchiole [28]. The total of all acini forms the lung parenchyma, the area where the pulmonary gas-exchange takes place.

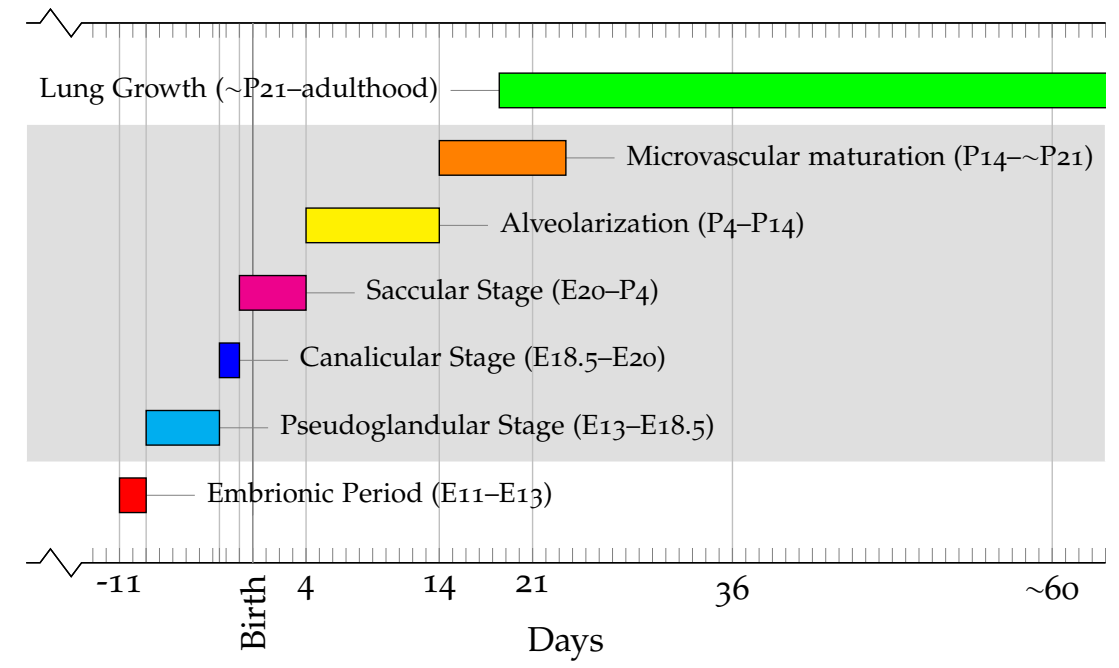

Figure 2.1: Lung development stages for the rat lung according to the classical paradigm. The first three stages (Embryonic Period, Pseudoglandular and Canalicular Stage) take place in the approximately 21 days from the beginning of gestation to birth. E=embryonic day (days post-coitus), $\mathrm{D}=$ postnatal day. The five stages mentioned in the text happen during the grey marked period. Note that the microvascular maturation and lung growth stages overlap each other by several days. All the other stages are drawn consecutively, but in reality they do slightly overlap, since as biological processes they change into each other. Figure adapted from [11].

The pseudoglandular stage is followed by intermediate stages, referred to as canalicular and saccular. During the canalicular stage a first functional gas exchange surface- - the air-blood barrier-is formed and the lung epithelium starts to differentiate. The saccular stage marks the switch from branching morphogenesis to the formation of the alveoli, or septation of the existing gas exchanging air spaces (Alveolarization stage).

During the alveolar stage the distal part of the bronchial tree is enlarged by the introduction of new alveoli through a formation of additional septa from already existing septa.

In parallel to alveolarization, the interalveolar septa and their capillary networks are remodeled during microvascular maturation in order to optimize gas exchange (personal communication with Prof. Dr. Johannes C. Schittny). At this point lung development is considered

The time point of birth differs between mammals, relative to

the state of lung development. In humans, birth happens at the beginning of the alveolarization stage. to be finished and normal growth of the organ follows.

It was believed that lung developments comes to completion during the early postnatal period due to the reduction of a double- to a singlelayered capillary network inside the alveolar septa. After this period, only growth of the organ should follow, and no additional alveolar septa are introduced in the lung parenchyma $[27,29]$. Schittny et al. [18] were able to refine this model with an additional development stage in which the so called late alveolarization-the formation of alve- 
olar septa until young adulthood (days $4-60$ in rats) -takes place. A local duplication of the capillary network was detected as the basis of these newly forming septa. Figure 2.2 shows the refined sequence of the lung development stages. Two alveolarization phases and the phase of microvascular maturation occur simultaneously after postnatal day four until postnatal day 60.

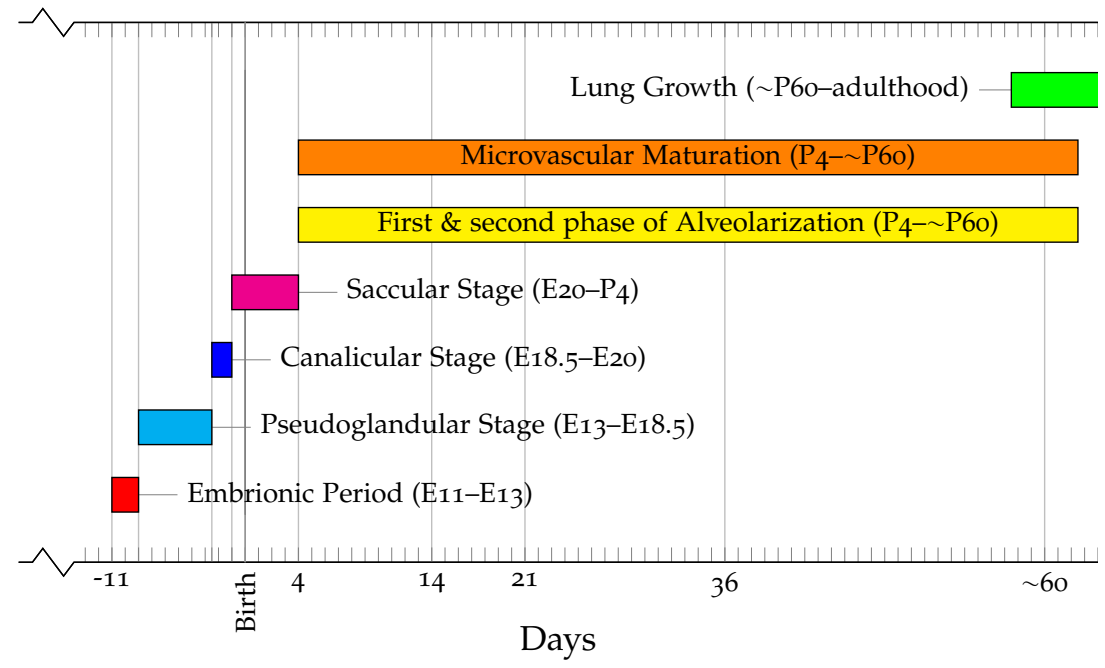

Figure 2.2: Lung development stages with concurrently occurring Alveolarization and Microvascular Maturation stages. E=embryonic day (days post-coitus), $\mathrm{D}=$ postnatal day. Note that alveolarization and microvascular maturation take place simultaneously. Lung growthgrowth without the formation of new alveoli-follows afterwards. Data for days $>60$ has not been recorded. Unpublished data from private communication with Johannes C. Schittny.

\subsection{THE FUNCTIONAL UNITS OF THE LUNG}

The airway structure of the human lung is formed from dichotomous branches, starting from the trachea. The first branching generations lead into the bronchi (Figure 2.3a). With increasing depth into the airway tree, the diameter of the airways is reduced, the bronchi are divided into bronchioles, leading to the terminal bronchioles which mark the end of the purely conducting airways. The respiratory bronchioles mark the start of the gas-exchange region in the lung, the point from which the so-called acinar airways and the acinus begin.

Ongoing from the respiratory bronchioles, the acinus is further subdivided into alveolar ducts, sacculi and alveoli, as shown in Figure 2.3b $[10,11,30,31]$. Note that Figure $2.3 \mathrm{~b}$ shows a representation of the generations in the human lung. Instead of several generations of respiratory bronchioles as in the human lung, rat lungs only have one generation of transitory bronchioles.

\subsection{THE NEED FOR TOMOGRAPHY}

In our group there has been an ongoing effort to analyze the parenchymal structure of the rat and mouse lung, two laboratory animals which are extensively used in respiratory research. Schittny et al. [18] challenged the model of alveolarization-where the gas exchange surface 


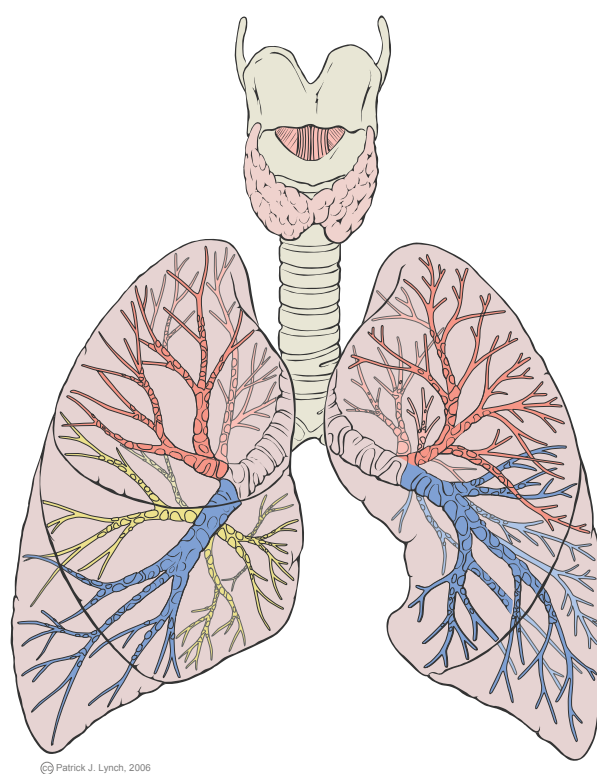

(a)

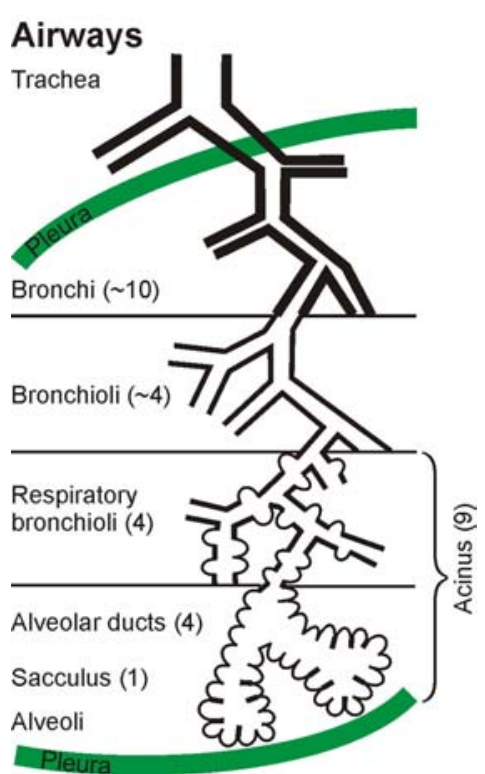

(b)

Figure 2.3: Details of the human lung. (a): Lung diagram of the human lung. From [32]. (b): Airway generations in the human lung. From [11]. Note that rat lungs have only one generation of transitory bronchioles instead of several generations of respiratory bronchioles.

is enlarged through the lift off of new septa from pre-existing septa [21, 33] - and developed a new theory on late alveolarization.

Traditionally, quantitative information and physical properties of the lung structure are obtained using stereology [34]. Stereology refers to the mathematical methods for analyzing these properties of an irregular three-dimensional structure using two-dimensional planar sections obtained by physical or optical imaging techniques [35].

Stereology is considered to be the standard method for unbiased analysis of morphological values in the lung like three-dimensional volume or size, two-dimensional surface area, one-dimensional length and thickness of structures or global number of alveoli. Nonetheless, certain parameters of the lung structure like the topological characteristics of the airway tree, the volume of a individual acinus and which alveolus is ventilated by which alveolar duct cannot be determined using stereological methods, but need analysis of the three-dimensional relations using tomographic data.

Additionally, the simulation of deposition of particles inside the lung based on the simulation of the airflow characteristics in the terminal airways is only possible with three-dimensional reconstructions of the lung tissue. For an extremely small sample volume of a duck lung Woodward and Maina [5] achieved interesting results using 60 serial sections with a thickness of $300 \mathrm{~nm}$ each. The digital reconstruction of this volume made it possible to clarify the three-dimensional configuration of the terminal respiratory units in the duck. The terminal airways were found to be roundish, heterogeneous structures which connect through narrow conduits, the blood capillaries were shown to form a network of interconnected segments, with approximately equal lengths and diameters. Nonetheless, the volume of such a reconstruc- 
tion of the terminal airways would be much too small for any of the simulations mentioned above.

Using tomographic microscopy, especially Synchrotron Radiation based X-ray Tomographic Microscopy (SRXTM) three-dimensional data of lung samples with a volume of $1.5 \times 1.5 \times 1.5 \mathrm{~mm}$ with a resolution high enough to resolve the alveolar septa can be acquired in a matter of minutes [36]. Since the three-dimensional data allows a fully unrestricted view into the terminal airways, such datasets are very well

Even larger volumes can be acquired with this resolution, see chapter 7 . suited to answer all the above mentioned questions.

The following chapter introduces the basic concepts for computed tomography and describes the machine which was used to obtain all tomographic data for the experiments presented in this thesis. 

More apparatus, please, nurse. Get the EEG, the BP monitor, and the AVV! And get the machine that goes "PING!" And get the most expensive machine in case the Administrator comes.

- John Cleese and Graham Chapman as Obstetricians in The Meaning of Life [37]

Computed Tomography (СТ) is an imaging method where a threedimensional image of the internal structure of an object is generated from a series of two-dimensional x-ray images-single radiographswhich have been taken at equidistant angles around a single rotation axis. The word tomography is derived from the greek words for tomos

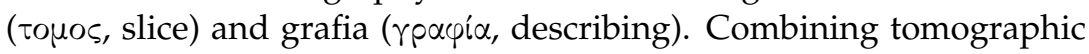
image acquisition with $x$-ray microscopy based on synchrotron radiation allows to obtain volumetric information of a specimen at submicron resolution with only minimal sample preparation [38] in a matter of minutes [36].

This chapter presents a short rundown on the history of CT and the underlying principles of the interaction of radiation with matter. Subsequently, the theory behind tomographic reconstruction is presented. The chapter ends with a short description of the machine that goes "PING!"- the beamline for TOmographic Microscopy and Coherent rAdiology experimenTs (TOMCAT) at the Swiss Light Source (SLS) in Villigen, Switzerland-where all experiments of this thesis have been performed.

\subsection{HISTORY}

Sir Godfrey Hounsfield presented the first commercially available tomograph in the 1970s [7], thanks to the upcoming availability of microcomputers. Since then, computed tomography has come a long way.

The basic idea of todays tomography was already described in a patent granted to Gabriel Frank [39] in 1942 and several experiments around 1960-1965 showed the feasibility of three-dimensional reconstruction of an object which has been penetrated by radiation [40]. The 
Sigi, this quote is especially for you!
The experiments presented in Part ii have been performed between $11.5 \mathrm{keV}$ and $12.6 \mathrm{keV}$. first tomographic imagers produced radiographic images of selected sections of tissue by moving the $x$-ray source and the film over and under the patient in the opposite direction. This approach blurred structures lying out of the selected region of interest. But this advance over conventional radiographies was insufficient to make two-dimensional tomographic images a useful diagnostic tool [41].

While observing the planning of radiotherapy treatments, Allan M. Cormack realized that these treatments would greatly benefit if the $\mathrm{x}$ ray attenuation coefficient distribution in the body would be known. In 1963 Cormack [42] reported about the investigation of a cylindrical disk with a diameter of $20 \mathrm{~cm}$ which was made of two concentric aluminum cylinders embedded in an oak annulus. Using a collimated ${ }^{60} \mathrm{Co}$ source and a Geiger counter as a detector, Cormack was able to detect the absorption coefficients of the materials in the cylinder and repeated this experiments with an asymmetrical phantom consisting of aluminum and plastic. But since the calculations necessary for the extraction of the attenuation coefficients were both time-consuming and difficult, the presented results never really caught on. Cormack remarked that

There was virtually no response. The most interesting request for a reprint came from a Swiss Centre for Avalanche Research. The method would work for deposits of snow on mountains if one could get either the detector or the source into the mountain under the snow! [43]

In 1967, Godfrey N. Hounsfield started to develop the first clinical tomography scanners at the EMI Central Research Laboratories in the United Kingdom [40]. The first laboratory scanners took nine (!) days to acquire a scan of one slice of a cow brain [44], but paved the path to the first clinically available CT machines in hospitals and the scientific community. In 1979 Cormack and Hounsfield shared the Nobel Prize for Physiology and Medicine for their pioneering work in CT.

From the early 1980 s on, CT imaging became an important tool to answer scientific questions both in medical and industrial applications. Even if these medical CT scanners are in widespread use, their images suffer from relatively low spatial resolution in the sub-millimeter scale, limiting their use for the detection of the finest details in biological samples. Since the radiation dose limitations imposed for medical scans often do not apply for biological samples which are dried, frozen or embedded in paraffin or plastic, more intense and collimated $\mathrm{x}$-ray sources can be used. Using such radiation sources in microComputed Tomography $(\mu \mathrm{CT})$ stations enables to reach resolutions in the sub-micrometer range [8]. Due to the fact that synchrotron radiation is even more powerful than the x-ray tubes uses for $\mu \mathrm{CT}$, Grodzins $[45,46]$ proposed the used of synchrotron sources for the production of highly intensive x-ray beams which were then used for microtomography applications.

\subsection{INTERACTION OF RADIATION WITH MATTER}

To be able to explain the physical principles of $\mathrm{CT}$, some important interactions of $x$-rays with matter have to be introduced. The energy of the x-ray photons available at TOMCAT, where all the experiments of this work have been performed is in the range of 8-45 keV, we can thus 
limit the explanation to absorption, Rayleigh and Compton scattering and neglect the description of electron-positron pair production, which occurs only for x-ray energies above $1 \mathrm{MeV}$.

\subsubsection{Absorption}

In the relevant absorption process-the so-called photoelectric effectan incoming x-ray photon with energy $E_{\gamma}$ interacts with a bound atomic electron in a deep shell of the atom and is completely absorbed. This process only happens if $E_{\gamma}$ is greater than the binding energy $E_{S h e l l}$ of the shell electron and releases a photo-electron with the energy $\Delta \mathrm{E}=\mathrm{E}_{\gamma}-\mathrm{E}_{\text {Shell }}$. This liberation creates a hole in the deep shell, which is immediately filled by electrons from outer shells of the atom. Since the electron from the outer shell is at a higher energy state than the inner shell electron, a cascade process is initiated which results in the emission of radiation which is characteristic for the absorbing atom.

\subsubsection{Scattering}

\subsubsection{Compton scattering}

Compton [47] first described an inelastic scattering effect which is observed when an incident photon with an energy $E=h v$ transfers parts of its energy to a scattering electron which is subsequently ejected from the atom.

To conserve the overall momentum of the system, the electron recoils at an angle $\theta$ and the photon is scattered in a direction $\phi$ with a reduced energy $E=h v^{\prime}$, where $h$ denotes the Planck constant. If the photon still has enough energy left, the process may be repeated, if the photon is of lower energy, it can subsequently be absorbed as described above in subsection 3.2.1. The reduction in the energy of the recoil electron leads to a shift in the wavelength $\Delta \lambda$ which can be calculated using Equation 3.1

$$
\Delta \lambda=\lambda^{\prime}-\lambda=\frac{h}{m_{e} c}(1-\cos \theta)
$$

where $m_{e}$ stands for the electron mass and $c$ for the speed of light.

\subsubsection{Coherent scattering}

Coherent or Rayleigh scattering is an interaction process where no ionization occurs and no energy is converted into kinetic energy. The incident electromagnetic wave sets the electrons in the magnetic field into vibration with an oscillating electric field. The oscillating electrons emit coherent radiation, but since not all electrons are located at the same point inside the atom, the emitted radiation is not in phase [40, 48].

Figure 3.1 shows the relative percentages of interaction for the different interaction types. Note that albeit pair production is shown in the plots, it has not been described, since it is only relevant for energies much higher than the energies used in the experiments in this thesis, performed around $12 \mathrm{keV}$.

We see that for the energies used in this work (dark grey stripe in Figure 3.1), the photoelectric effect is the major interaction process, even if 


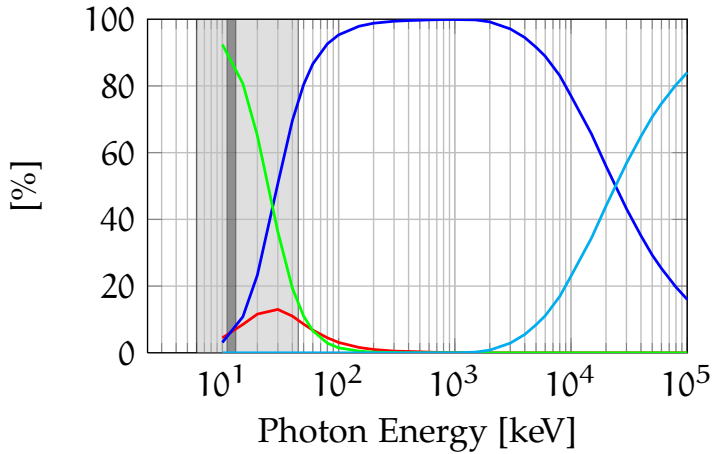

— Coherent (Rayleigh) scattering

- Incoherend (Compton) scattering

- Photoelectric absorption

- Pair production

(a) Overview

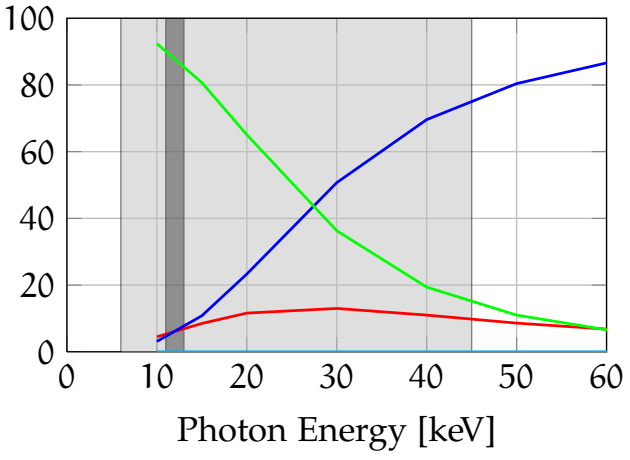

- Coherent (Rayleigh) scattering Incoherend (Compton) scattering Photoelectric absorption Pair production

(b) Detailed view

Figure 3.1: Percentage of the different interaction types specified above as a function of the beam energy in water. (a): Overview over the full given energy range. (b): Detailed view. The lightgray region is the energy-range of TOMCAT, the dark gray region marks the energy range of the experiments described in chapters $5-7$ of this thesis. Data from Johns and Cunningham [49, Table 5-5].

its amount rapidly decreases for higher energies. Not only is the interaction rate higher for the photoelectric effect, but also the major part of the beam energy is transferred, since more energy is transferred in a photoelectric interaction than in a Compton interaction.

\subsubsection{Attenuation}

The total effect of the three effects described above can be summarized with the total linear attenuation coefficient shown in Equation 3.2.

Monochromatic light is light of a single wavelength or energy, though in practice it often refers to a narrow wavelength range This equation expresses the attenuation of a monochromatic beam as it passes through a material. The attenuation in a material of uniform density and atomic number can be expressed by an exponential relationship

$$
\mathrm{I}=\mathrm{I}_{\mathrm{O}} \mathrm{e}^{-\left(\tau+\sigma_{\mathrm{C}}+\sigma_{\mathrm{R}}\right) x}
$$

where $I$ and $I_{0}$ are the transmitted and incident intensities of the $x$ rays and $x$ is the distance which the $x$-rays travel through the material. $\tau, \sigma_{C}$ and $\sigma_{R}$ are the attenuation coefficients of the photoelectric, incoherent (Compton) and coherent (Rayleigh) scattering interactions in the material, respectively.

The so-called Beer-Lambert law as shown in Equation 3.3 summarizes the single attenuation coefficients into one attenuation coefficient $\mu$, depending on the material and on the photon energy of the incident $\mathrm{x}$-ray beam [40]:

$$
\mathrm{I} / \mathrm{I}_{\mathrm{O}}=\mathrm{e}^{-\mu x}
$$

We can thus calculate the fraction of the transmitted to the incident beam intensities knowing the travel distance of the beam through the material and the attenuation coefficient of said material. 
As an example, Figure 3.2 shows the composition of the attenuation coefficient for Cerium doped Yttrium Aluminium Garnet (YAG) $\left(\mathrm{Y}_{3} \mathrm{Al}_{5} \mathrm{O}_{12}\right)$, which can be used as a material for converting the incident X-rays to visible light. Ce:YAG is used as a so-called scintillator as a part of the microtomography station at TOMCAT which is described in detail in section 3.6.

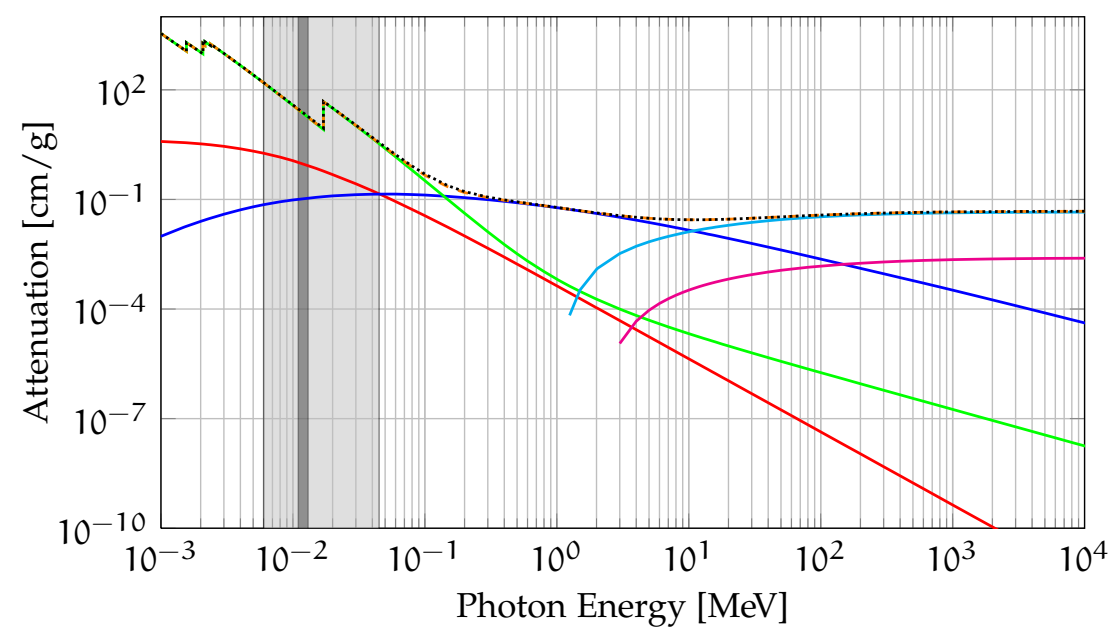

$$
\begin{aligned}
& \text { - Coherent (Rayleigh) scattering } \\
& \text { - Incoherend (Compton) scattering } \\
& \text { - Photoelectric absorption } \\
& \text { - Pair production in nuclear field } \\
& \text { - Pair production in electron field } \\
& \text { - - - Total attenuation without coherent scattering } \\
& \text {-....... Total attenuation with coherent scattering }
\end{aligned}
$$

Figure 3.2: Total attenuation coefficient of Ce:YAG $\left(\mathrm{Y}_{3} \mathrm{Al}_{5} \mathrm{O}_{12}\right)$, the scintillator material used for converting the incident $\mathrm{x}$-rays to visible light in the experiments presented in chapters $5-7$. The lightgray region is the energy-range of TOMCAT, the dark gray region marks the energy range of the experiments described in chapters chapter $5^{-7}$ of this thesis. Data from the photon cross section database [50].

\subsection{FROM PROJECTIONS TO SLICES: COMPUTED TOMOGRAPHY}

In general, $\mathrm{CT}$ refers to the cross-sectional imaging of a sample from transmission images or projections recorded at several equiangular positions around the sample [51].

In medical CT-scanners, the transmission images are generally obtained while the radiation source and the detector rotate around the body part to be imaged. In micro-computed and synchrotron based tomography, the source and detector are fixed, while the sample to be imaged rotates around a chosen axis.

During the imaging process multiple transmission images-which are essentially single radiographic images-are obtained at several equidistant angles over a $180^{\circ}$ or $360^{\circ}$ rotation. These projections can be understood as pictures of the distribution of the attenuation of the $x$-rays inside the sample. Regions with high attenuation (e.g. tissue) show up as dark pixels, regions with low attenuation (e. g. airway lumens) show up as light pixels on the CCD-chip of the camera. 
Before and after the scan, so-called dark $\left(\mathrm{I}_{\mathrm{D}}\right)$ and flat images $\left(\mathrm{I}_{\mathrm{F}}\right)$ are recorded. The dark images record the camera noise and dark current, while the flat images record the beam profile. After baseline correction of the projections $\left(I_{P}\right)$ the average of the dark images is subtracted. The projections are then normalized to the flat images as seen in Equation 3.4, using the average dark and average flat images $\left(\overline{I_{D}}\right.$ and $\bar{I}_{F}$, respectively) resulting in so-called corrected projections (CPR).

$$
\mathrm{CPR}=-\ln \left(\frac{\mathrm{I}_{\mathrm{P}}}{\overline{\mathrm{I}_{\mathrm{F}}}}\right)=\ln \left(\overline{\mathrm{I}_{\mathrm{F}}}\right)-\ln \left(\mathrm{I}_{\mathrm{P}}\right)
$$

Figure 3.3 shows the results of correcting a raw projection obtained at TOMCAT with a flat image into a corrected projection.

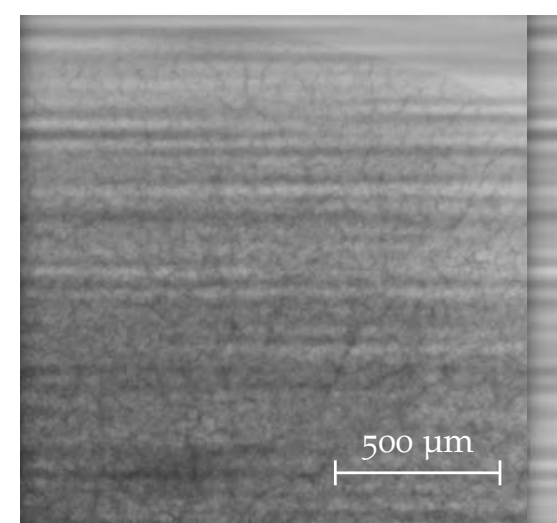

(a) Projection $I_{P}$

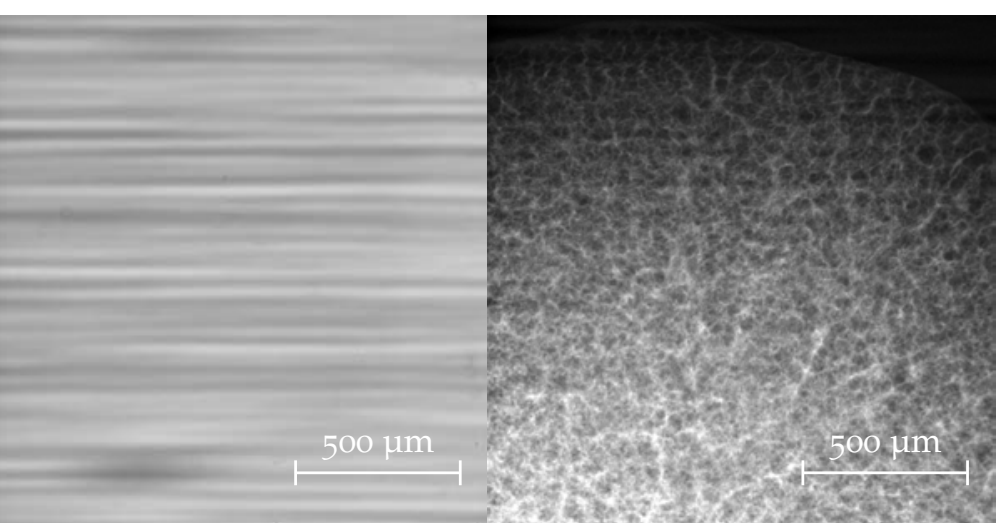

(b) Flat Image $I_{F}$

(c) Corrected projection CPR

Figure 3.3: Correction of the raw recorded projections with the flat images. (a): Recorded projection image. A set of these is the output of one tomographic scanning session. (b): Flat image; obtained at the start of the scan to record the beam profile. (c): Corrected projection obtained after Equation 3.4 has been applied on the projection and flat image shown in panels (a) and (b). All three images have a size of $1024 \times 1024$ pixels with a size of $1.48 \mu \mathrm{m}$ each and are from a dataset of a rat lung sample obtained at postnatal day eight.

The corrected projections are then transformed into so-called sinograms, where the $\mathrm{n}^{\text {th }}$ sinogram is composed of the $\mathrm{n}^{\text {th }}$ line of each projection. From one tomographic scan with 1500 projections recorded with a detector with a size of $2048 \times 2048$ pixels, we get a set of 1024 sinograms with a size of $1024 \times 1500$ pixels each.

The Radon

Sinograms inherit their name because the Radon transformation of a Dirac delta peak resembles a sine wave. The Radon transformation of a cluster of objects appears as an overlay of blurred sine waves with different amplitudes and phases, as shown in Figure 3.4a, since any object can be considered as a collection of many small points.

One sinogram contains the total information of one plane or slice of the reconstructed tomographic image. We can thus reconstruct the $n^{\text {th }}$ slice of the tomographic dataset from the $n^{\text {th }}$ sinogram using different reconstructing algorithms, e.g. a standard filtered back-projection algorithm. In a reconstructed slice, the absorption properties of the sample at a certain height are encoded by the gray values of the image, as shown in Figure 3.4b. This virtual slice into a rat lung sample is part of a dataset permitting a fully three-dimensional, unrestricted view into the sample in a non-destructive way. 


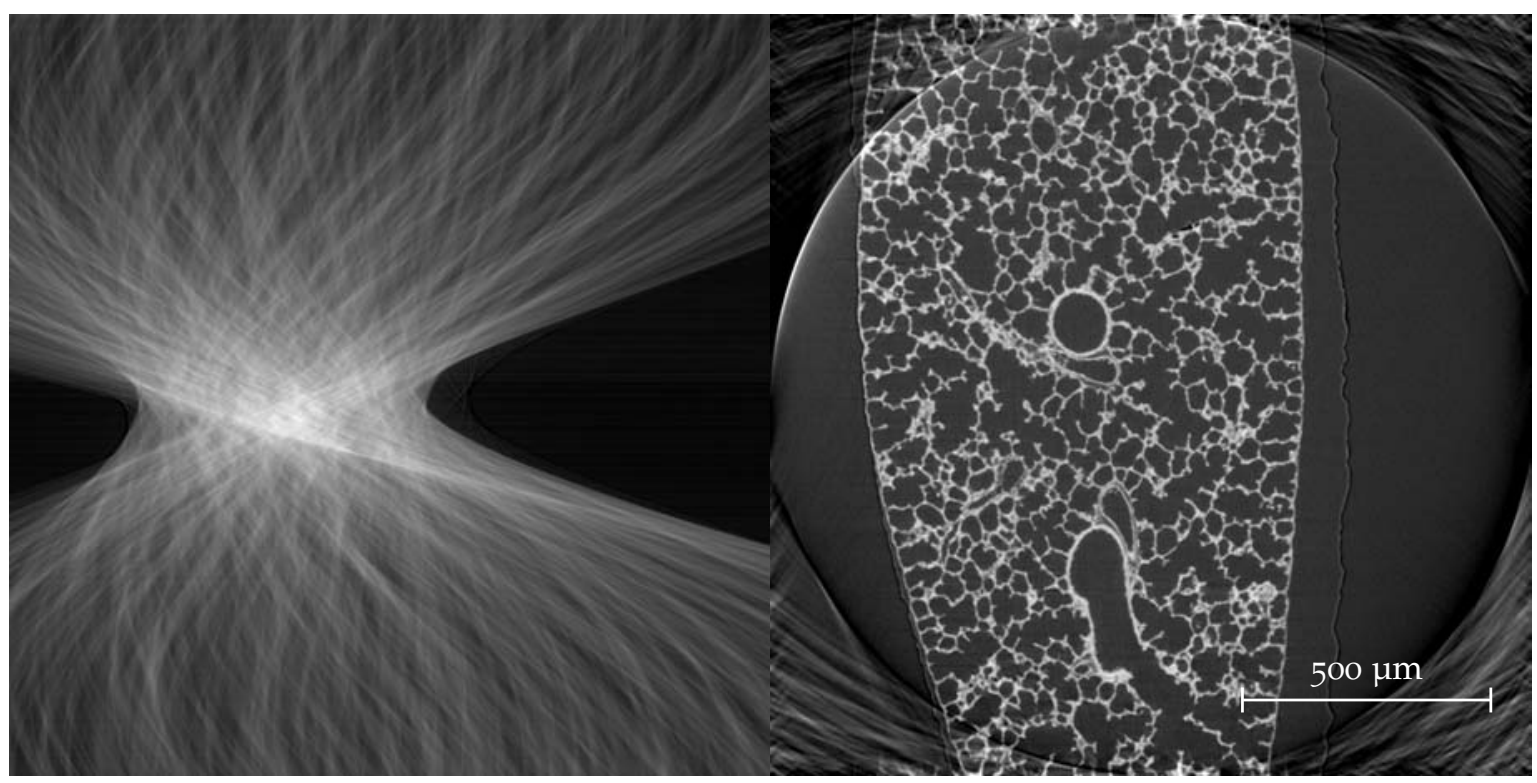

(a) resized Sinogram

(b) Reconstructed Slice

Figure 3.4: Sinogram and Reconstruction of the projections of the rat lung sample shown in Figure 3.3. (a): The $n^{\text {th }}$ sinogram has been obtained after reordering the $n^{\text {th }}$ line of each of the 1501 corrected projections. The sinogram has been rescaled to a square format for display purposes, it would have a size of $1024 \times 1501$ pixels. (b): The tomographic reconstruction of the complete set of sinograms results in a set of 1024 reconstructed slices with $1024 \times 1024$ pixels of a size of $1.48 \mu \mathrm{m}$ each. A set of such slices is then used for further processing, measuring and three-dimensional visualization.

The amount of scanned projections-the number of angles at which projections have been recorded-is defined by the so-called sampling theorem. The sampling theorem as defined by Shannon [53] states that:

If a function $f(t)$ contains no frequencies higher than $W$ $\mathrm{cps}$, it is completely determined by giving its ordinates at a series of points spaced $1 / 2 \mathrm{~W}$ seconds apart. [53]

Paraphrased to tomographic imaging, the sampling theorem states that the minimal number of recorded angles along the rotation of the sample must be at least equal to twice the highest frequency used to scan the sample.

From calculations in polar coordinates one can deduce that for a detector with the size $N M=\frac{\pi}{n} N$ angular projections are required to fulfill the sampling theorem for tomographic reconstruction [54]. Generally, for well-balanced reconstructed image with a size of $\mathrm{N} \times \mathrm{N}$ pixels, recorded with a detector of the same size, the total number of projections should be roughly equal to N [51]. In layman's terms, we can thus say that if we scan a sample with a detector of $1024 \times 1024$ pixels, we need to record approximately 1000 projections over a sample rotation of $180^{\circ}$.

At TOMCAT this rule of thumb is exactly obeyed in the case of a tomographic scan with a binned detector. For a standard scan with an unbinned detector-resulting in images with a size of $2048 \times 2048$ 
pixels-only 1500 projections are acquired, thus violating the rule. But even with this seemingly undersampled data acquisition, reconstructions with very high quality are obtained.

chapter 7 presents a scanning method where the sampling theorem is deliberately violated both to increase the field of view of TOMCAT and to reduce the radiation dose imparted on the sample.

For classic tomographic imaging, an $\mathrm{x}$-ray tube is used as a source of radiation. X-rays from a synchrotron are used as a radiation source for Synchrotron Radiation based X-ray Tomographic Microscopy (SRXTM), which was used to obtain the tomographic data for this thesis. The following sections give a short overview over the underlying theory of the synchrotron radiation and a short rundown on the SLS and the beamline where all the presented experiments have been carried out.

\subsection{SYNCHROTRON RADIATION}

Synchrotron facilities use the fact that accelerated particles traveling on a curved trajectory emit radiation. If charged particles at relativistic speed undergo a change of direction (i. e. in a magnetic field) so-called synchrotron radiation is emitted.

Synchrotron radiation was first observed as energy loss in electron storage rings used for high energy physics experiments. First generation synchrotron sources for scientific use were beamports at such storage ring facilities, which utilized otherwise lost radiation. Dedicated second-generation synchrotron radiation sources have been built over the years; these were ring-like structures using a series of magnets to control the accelerated particles. Current third-generation synchrotron radiation facilities are composed of many straight sections of different lengths, specially optimized to accommodate different magnetic structures used to generate the synchrotron radiation [54-56].

\subsubsection{Bending Magnets, Undulators and Wigglers}

The three magnetic structures used in synchrotron facilities are bending magnets, undulators and wigglers. All three emit radiation with different characteristics and intensities.

Such magnets are also used in traditional

televisions, which contain a cathode ray tube, which is essentially a small particle accelerator. They move the electron beam over the screen of the TV tube in a controlled fashion. [57]
Bending magnets are used to create a homogeneous magnetic field over a defined distance. The magnetic field inside forces accelerated particles that are injected into the bending magnet to travel on a circular trajectory. The result is a fan of radiation in the tangential direction of the bend.

Undulators consist of periodic structures of dipole magnets with relatively weak fields. The alternating static magnetic field forces the electrons to harmonically oscillate as they move in the axial direction, resulting in an undulating motion of the particles inside the structure. The weak magnetic field causes small amplitude undulations which lead to a narrow radiation cone. Through coherent addition of the tightly confined electron beam, the produced radiation is emitted with small angular divergence and concentrated in narrow energy bands [54].

Wigglers are the strong brothers of the undulators. Due to stronger magnetic fields the oscillation amplitude of the electrons and the emitted radiation power are larger and the radiation cone is broader. 


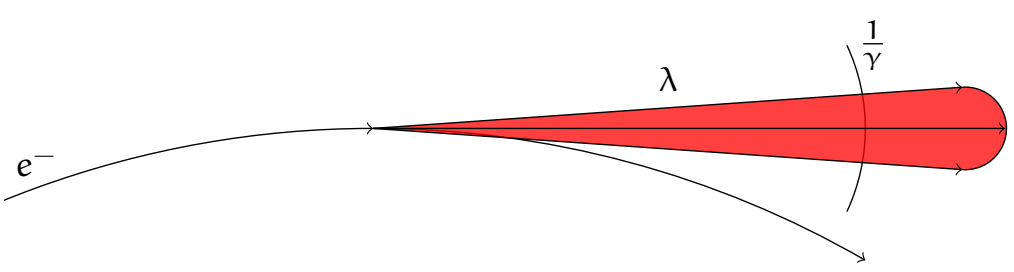

(a) Beam

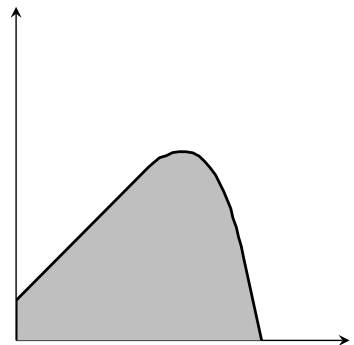

(b) Spectrum

Figure 3.5: Bending magnet radiation of wavelength $\lambda$ produced by a relativistic electron traveling in an uniform magnetic field. (a): Electrons execute a circular motion with acceleration directed towards the center. The radiation is directed tangentially outward in a narrow radiation cone with an emission angle of typically $\frac{1}{\gamma}$ where $\gamma$ is the Lorentz contraction factor. (b): The radiation spectrum is very broad, analogous to a white light $\mathrm{x}$-ray light bulb. Adapted from [58].

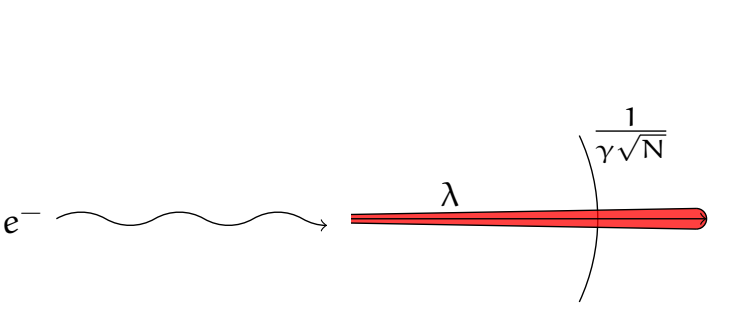

(a) Beam

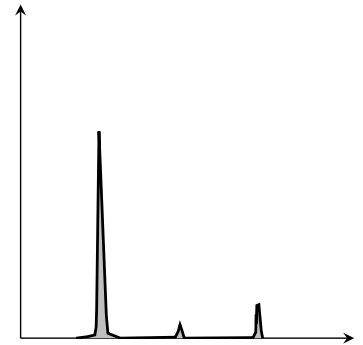

(b) Spectrum

Figure 3.6: Undulator radiation is generated as a highly relativistic electron traverses a periodic magnetic field. (a): The magnetic field in an undulator is relatively weak and the resultant angular excursions of the electron are smaller than the angular width of the natural radiation cone, $\frac{1}{\gamma}$, normally associated with synchrotron radiation. (b): The frequency spread of undulator radiation can be very narrow, and the radiation can be extremely bright and partially coherent, under certain circumstances. The characteristic emission angle is narrowed by a factor $\sqrt{N}$, where $N$ is the number of magnetic periods. Typically $\mathrm{N}$ is of order 100. Depending on the magnet strength, harmonic radiation may be generated. Adapted from [58]. 


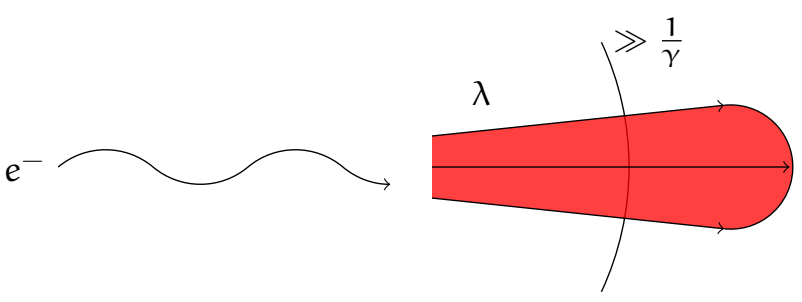

(a) Beam

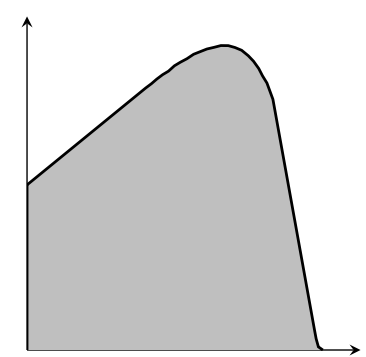

(b) Spectrum

Figure 3.7: Wiggler radiation is also generated from a periodic magnet structure. (a): In the strong magnetic field the angular excursions are significantly greater than the natural $\left(\frac{1}{\gamma}\right)$ radiation cone. Because accelerations are stronger in this limit, the radiation generated peaks at higher photon energies and has both a higher photon flux and more power. (b): The radiation spectrum is very broad, similar to that of the bending magnet. Although more power is radiated, wiggler radiation is less bright because of the substantially increased radiation cone. Adapted from [58].

The relativistic electrons inside the storage ring are usually produced by a hot filament and subsequently pre-accelerated and injected into the storage ring by a linear accelerator. Most third-generation synchrotron light sources are operated in the so-called top-up mode, where a steady current in the storage ring is maintained through periodical injection of small amounts of current from a booster ring or directly from the linear accelerator.

Bending magnets along the storage ring keep the electrons on their circular path. Quadrupole magnets along the ring are used to improve the geometrical characteristics of the electron beam, i. e. reduce the transverse section and angular spread of the beam, hence improving the so-called brightness of the source [55].

Using one of the three above mentioned structures, the radiation is then outcoupled of the storage ring and transferred into the measuring hut. At TOMCAT the radiation is outcoupled using a very strong bending magnet, a so-called superbend, which is described in detail below.

\subsection{THE SWISS LIGHT SOURCE}

The Swiss Light Source at the Paul Scherrer Institut (PSI) in Villigen, Switzerland is a third-generation synchrotron light source. With an energy of $2.4 \mathrm{GeV}$, it provides photon beams of high brightness for research in materials science, biology and chemistry. The donut-shaped SLS building has an outer diameter of $138 \mathrm{~m}$, an inner diameter of $32 \mathrm{~m}$ and a height of $14 \mathrm{~m}$.

\subsection{TOMСАТ}

At the beamline for TOmographic Microscopy and Coherent rAdiology experimenTs (TOMCAT) the user can perform absorption as well as phase contrast imaging with an isotropic voxel size ranging from $350 \mathrm{~nm}$ up to $14.8 \mu \mathrm{m}$, depending on the chosen magnification. Typi- 


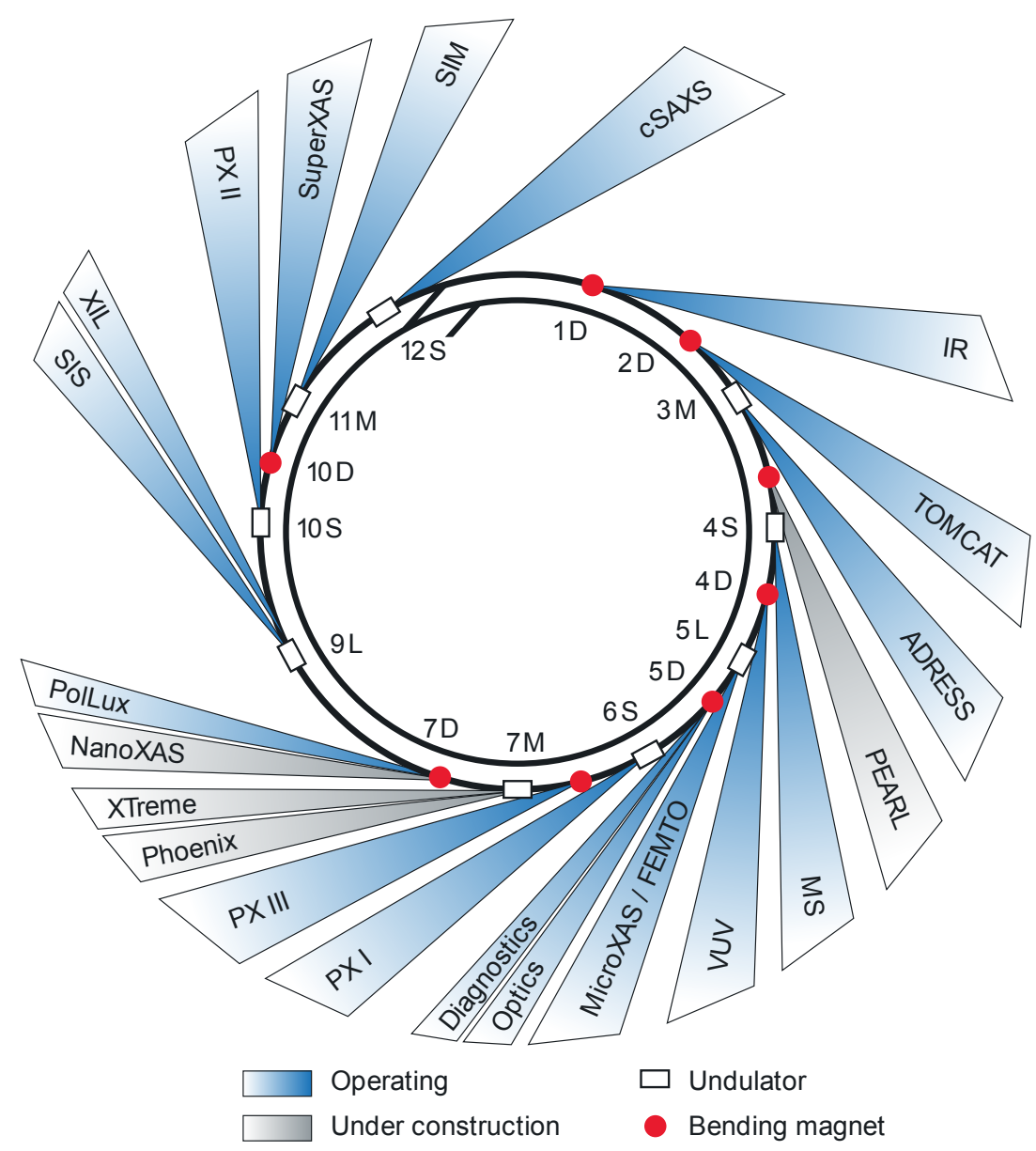

Figure 3.8: Beamlines at the SLS. Currently, 17 beamlines are in operation while four beamlines are under construction. Image from the SLS Website [56] (with permission).

cal acquisition times are in the order of a few minutes for a full sample, depending on the selected energy and resolution.

Stampanoni et al. [38] described the TOMCAT beamline in detail, a short rundown on the most important features are presented here: The beamline is located at the $\mathrm{X}_{02} \mathrm{DA}$ port of the SLS and started regular user operation in June 2006. Synchrotron light is delivered by a $2.9 \mathrm{~T}$ bending magnet with a magnetic field more than double the strength of the normal sLS bending magnets. This enables to have a high critical energy of the source (11.1 keV, corresponding to a wavelength of $1.22 \AA$ ) and results in a considerable increase of flux at the desired energy range.

Because of the high coherence of the used synchrotron radiation is desired to be kept until the radiation penetrates the sample, a minimization of the optical elements was targeted during the design of the beamline. A $100 \mu \mathrm{m}$ thick diamond window separates the ultra high vacuum $\left(10^{-10} \mathrm{mbar}\right)$ from the vacuum section of the beamline ( $\left.{ }^{-7} \mathrm{~m}^{-7} \mathrm{mbar}\right)$. After the beam passes the diamond window, its energy is selected using a double crystal multilayer monochromator (Strumenti Scientifici CINEL s.r.l., Padova, Italy). This main optical component of the beamline covers an energy range of 6-45 $\mathrm{keV}$ with a bandwidth of a few percent down to a few permille [38].

Flux is defined as the amount of particles, mass or energy which flows through an area in a certain time and essentially describes the brightness of the beam 
The latin word scintillare can be translated with sparkling or flickering.

See chapter 7 for a method to increase the field of view of tomography endstations while keeping the small voxel size.
After collimation, the beam travels through an evacuated pipe into the measuring hut and is directed towards the sample, as shown in Figure 3.9a. The sample sits atop a high precision sample manipulator (resolution better than $1 \mu \mathrm{m}$, the manipulator is shown in Figure $3.9 \mathrm{~b}$ and 3.9c) which is used for centering the sample in the beam and for the rotation of the sample for tomographic imaging.

The x-rays which penetrated the sample are detected using a combination of a scintillator, microscope optics and a CCD-camera as shown in Figure 3.9d. The scintillator converts incident x-rays to visible light through fluorescence. Charged particles excite the scintillator material and this excitation energy is then subsequently emitted by fluorescence photons at a longer wavelength, which makes it possible to detect the incident beam using a CCD-camera. The camera at TOMCAT features a detector with $2048 \times 2048$ pixels with a size of $7.4 \times 7.4 \mu \mathrm{m}$ each and can read out a full frame 14 bit image in $260 \mathrm{~ms}$. Between the scintillator and the CCD-sensor interchangeable microscope objectives enable to easily vary the pixel size range of the resulting tomographic reconstructions from $350 \times 350 \mathrm{~nm}$ up to $5.6 \times 5.6 \mu \mathrm{m}$. Depending on the chosen pixel size, the resulting field of view of a standard scan varies between $0.75 \times 0.75 \mathrm{~mm}^{2}$ and $11.45 \times 11.45 \mathrm{~mm}^{2}$.

The sample position in the beam and the parameters of the scanlike exposure time, number of projections, name, etc.- -are controlled from outside the measuring hut through an Experimental Physics and Industrial Control System (EPICS).

After the scan has been started, sinograms are calculated on the fly and the tomographic reconstruction can be initialized using a webbased interface seconds after the scan has been finished. This interface and the general reconstruction workflow and management of the reconstruction cluster has been described by Hintermüller et al. [36].

The web-based interface is used to submit the reconstruction of the tomographic datasets to a computing cluster of seven 64 bit Opteron machines with four cores and 8 GB RAM each. Immediately after reconstruction on the cluster the resulting data is copied to an external disk which makes it possible that the recorded tomographic datasets can be taken to the home laboratory immediately after the beamtime shifts.

This is where the real work begins... 


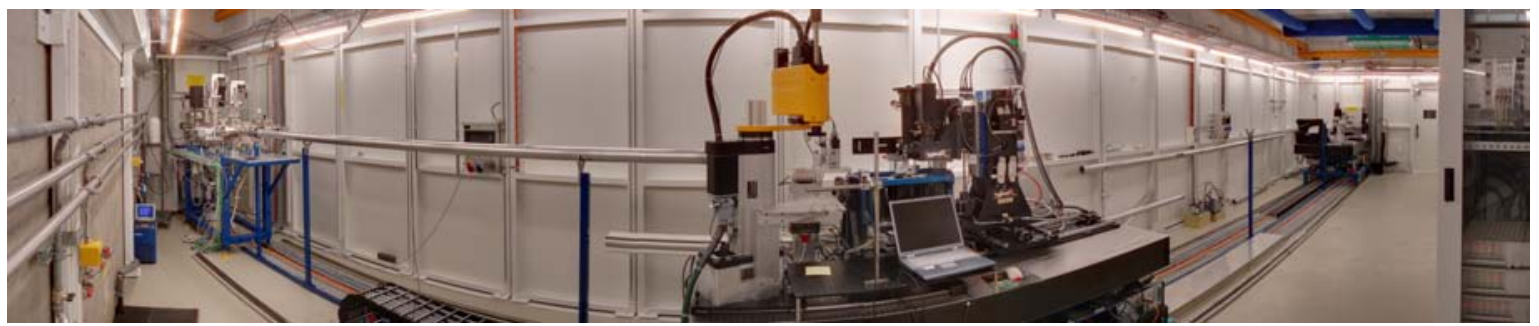

(a) Panoramic view

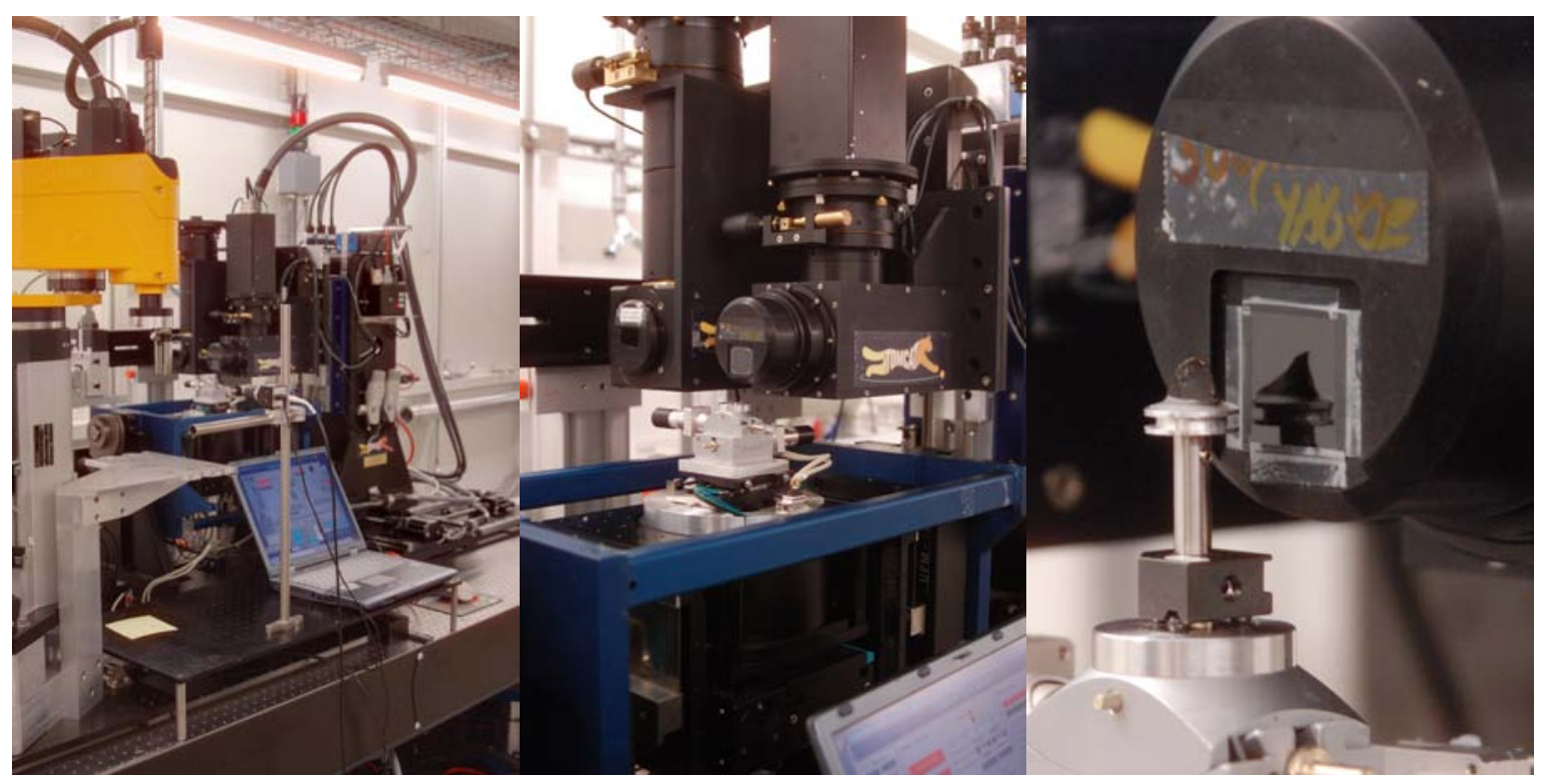

(b) Overview

(c) Detail

(d) Sample

Figure 3.9: The TOMCAT beamline. (a): $180^{\circ}$ panoramic view. The pipe in the center of the image in which the radiation is projected into the measuring hut is actually perfectly straight, it is just bent in this panoramic image. (b): Overview: The blue structure behind the control notebook is the sample stage with the sample holder on the rotation stage on top of it. The black structure above contains the scintillator, the microscope optics and the camera. The yellow contraption is the sample positioning robot used for unattended measurements. (c): Detail of the microscope optics: The sample rotation stage is visible in the middle of the image. The round black structure just behind the sample rotation stage contains the microscope objectives. The rectangle in the lower part of the objective revolver is the scintillator which is used to convert the x-ray beam into visible light, which after magnification is then recorded with the CCD-camera. (d): Rat lung sample on top of sample holder reflected in the scintillator. The standard scanning electron microscopy table with the mounted sample on top has a diameter of $12 \mathrm{~mm}$. 

Part II

RESULTS 

I'm throwing rocks tonight. Mark it, Dude.

- Steve Buscemi as Donny in The Big Lebowski [59]

The three chapters 5, 6 and 7 of this document are copies of publications I have authored or collaborated on. They represent the published results achieved during the time frame of this thesis.

\section{I CHAPTER 5 - MULTimodal IMAGiNG}

chapter 5 contains a proceeding that has been published in the Conference Series of the Journal of Physics after the $9^{\text {th }}$ International Conference on X-Ray Microscopy in Zürich, Switzerland. The manuscript presents a method for localization and three-dimensional imaging of sub-micron gold particles in rat lungs. Two batches of gold particles with sizes of $200 \mathrm{~nm}$ and $700 \mathrm{~nm}$ have been applied to rat lung samples through intratracheal instillation. The samples have been scanned with high resolution Synchrotron Radiation based X-ray Tomographic Microscopy (SRXTM) at the TOmographic Microscopy and Coherent rAdiology experimenTs (TOMCAT) beamline of the Swiss Light Source (SLS) with a voxel size of $350 \mathrm{~nm}$. This resolution was sufficient to detect the exact three-dimensional localization of single and clustered gold particles with a size of $700 \mathrm{~nm}$ in alveoli, alveolar ducts and terminal bronchioli in the rat lung samples.

As the particles with a diameter of $200 \mathrm{~nm}$ were below the detection limit of TOMCAT, we were only able to accurately localize them by Transmission Electron Microscopy (TEM). For this, the samples was sectioned into $300 \mathrm{~nm}$ thick sections and analyzed using TEM. We were able to verify the exact locations of the gold particles of both sizes in the lung tissue. Gold particles with a size of $700 \mathrm{~nm}$ were localized in consecutive sections even if they were not directly visible on certain slices. Since these particles were pulled out of the resin, we sometimes only observed a hole were gold particles would have been expected.

The high correlation between the virtual sections of the tomographic dataset and the real sections imaged through TEM makes the combi- 
Stereology refers to the mathematical methods for defining physical properties of irregular three-dimensional structures using two-dimensional sections obtained by physical or optical imaging techniques [35]. nation of both imaging modalities very well suited to obtain the full unrestricted $3 \mathrm{D}$ access using SRXTM and to verify the localization of the particles in the $3 \mathrm{D}$-space with very high resolution using TEM.

\subsection{CHAPTER 6 - FE 3D RECONSTRUCTION OF THE ACINUS}

chapter 6 presents a new image processing method for accurate threedimensional reconstruction of the gas exchange region of the lung. The method has been published in the Journal of Applied Physiology and is based on a Finite Element $(\mathrm{FE})$ analysis of the tomographic datasets recorded at TOMCAT.

The datasets have been segmented using an iteratively determined threshold. This threshold was chosen to match computed morphological parameters of the resulting three-dimensional reconstruction with the stereologically determined real morphological parameters of samples from the same lungs.

In this work we have been able to highlight numerous advantages of a three-dimensional analysis of the reconstructions over traditional stereology. One of the most fundamental advantages of the tomographic analysis is the non-destructive method of obtaining the informations. Using traditional methods, the sample has to be cut into histological sections, which essentially destroys the integrity of the sample. Using the tomographic dataset, a thorough analysis of the inner structure of sample can also be carried out on slices, but these slices are virtual. Another advantage of the virtual slicing through the sample is that slices with arbitrary orientation can be generated throughout the sample as long as needed, and interesting regions can be iteratively improved.

\subsection{CHAPTER 7 - EXPANSION OF THE FIELD OF VIEW}

In chapter 7 I present the so-called wide field scanning method developed in order to cover a larger field of view at TOMCAT. This larger field of view was needed to enable the three-dimensional analysis of the functional lung unit, the so-called acinus which seems to be growing to a larger extent than previously described [33, 60].

Additionally, since the smallest structures we need to unambiguously detect are the alveolar septa with a thickness of several micrometers, we need to obtain tomographic scans with large magnifications, i. e. high resolution in the order of one micron.

Generally, those two points can not be satisfied with a single tomographic scan, since an increase in the field of view can only be acquired with low magnification and vice-versa. The method presented in this chapter overcomes this limitation through merging of several high resolution scans to one scan overlapping a large field of view. Additionally—through optimization of the acquisition protocol-the scanning time is minimized, which greatly reduces the radiation dose inflicted on the sample, which is a first step towards in-vivo SRXTM at TOMCAT.

This so-called Wide Field Synchrotron Radiation Based X-ray Tomographic Microscopy (WF-SRXTM) was implemented as a proof of concept during my post-graduate master thesis of the Master of advanced Studies ETH in Medical Physics in the TOMCAT group. In the following months, the method has been refined into a scanning protocol and is 
now routinely applied to obtain tomographic datasets of rat lung samples with both large field of view and high resolution. This paper is currently in press and will be published in the Journal of Synchrotron Radiation. 

MULTIMODAL IMAGING FOR THE DETECTION OF SUB-MICRON PARTICLES IN THE GAS-EXCHANGE REGION OF THE MAMMALIAN LUNG

\author{
David Haberthür ${ }^{1}$ \\ Manuela Semmler-Behnke ${ }^{2}$ \\ Shinji Takenaka ${ }^{2}$ \\ Wolfgang G. Kreyling ${ }^{2}$ \\ Marco Stampanoni 3,4 \\ Akira Tsuda ${ }^{5}$ \\ Johannes C. Schittny ${ }^{1,6}$ \\ First published in: J. Phys.: Conf. Ser. 186012040 (3pp) \\ doi:10.1088/1742-6596/186/1/012040
}

\title{
5.1 ABSTRACT
}

The deposition sites of inhaled aerosols in the gas-exchange region of the lung represent one of the key parameters needed for the understanding of the interaction between these particles and lung tissue. In order to develop a method for three-dimensional imaging of sub-micron particles in lung tissue we applied gold particles (200 and $700 \mathrm{~nm}$ ) to rat lungs by intratracheal instillation. The samples were scanned at the beamline for TOmographic Microscopy and Coherent rAdiology experimenTs (TOMCAT) at the Swiss Light Source. The $200 \mathrm{~nm}$ particles were slightly below the detection capabilities of TOMCAT. Therefore, their localization was obtained only by electron microscopy. At a voxel size of $350 \mathrm{~nm}$ we observed single and clustered gold particles (70o $\mathrm{nm}$ ) in alveoli, alveolar ducts, and small bronchioli. The locations of the gold particles were verified by transmission electron microscopical serial sections. We observed a very high correlation between these two imaging modalities. We conclude that a combination of x-ray tomographic microscopy and electron microscopy allows the full unrestricted $3 \mathrm{D}$ localization of particles smaller than the resolution of $x$-ray tomographic microscopy. We are planning to use this method for the verification of the simulation of particle deposition in the airway tree.

\footnotetext{
I Institute of Anatomy, University of Bern, Bern, Switzerland

2 Institute for Inhalation Biology, Helmholtz Zentrum München, Germany

3 Swiss Light Source, Paul Scherrer Institut, Switzerland

4 Institute for Biomedical Engineering, University and ETH Zürich, Switzerland

5 Physiology Program, Harvard School of Public Health, US

6 schittny@ana.unibe.ch
} 


\subsection{OBJECTIVE}

To study the deposition of sub-micron particles in the mammalian lung, we used Synchrotron Radiation based X-ray Tomographic Microscopy (SRXTM) to record tomographic images of lung samples with high resolution (350 $\mathrm{nm}$ ). In addition to SRXTM, we used conventional Transmission Electron Microscopy (TEM) to obtain high resolution images (resolution smaller than $1 \mathrm{~nm}$ ).

\section{$5 \cdot 3$ MATERIALS AND METHODS}

We applied $200 \mathrm{~nm}$ and $700 \mathrm{~nm}$ gold particles to rat lungs by intratracheal instillation. Thirty minutes after instillation, the lungs were fixed with $2.5 \%$ glutaraldehyde by vascular perfusion, stained according to standard protocols for TEM and embedded in Epon. The samples were shaped to cylinders with a diameter of either 0.6 or $1.2 \mathrm{~mm}$ and a length of several millimeters using a watchmakers lathe and then scanned at TOMCAT [61], the beamline for TOmographic Microscopy and Coherent rAdiology experimenTs at the Swiss Light Source at the Paul Scherrer Institute in Villigen, Switzerland. The samples were scanned at a wavelength of $11.5 \mathrm{keV}$, post processed on a 20-node Linux cluster using filtered backprojection, resulting in an image stack of $2048 \times 2048 \times 2048$ pixels with isotropic voxels of $350 \mathrm{~nm}$ side length. The samples were visualized in three dimensions using an isosurface computed with Imaris x64 5.72 (Bitplane AG, Switzerland) on an AMD 64-based windows computer [19]. After tomographic image acquisition serial sections of the sample were cut and processed for transmission electron microscopy [17].

\section{$5 \cdot 4$ RESULTS}

We have been able to observe single and clustered gold particles in alveoli, alveolar ducts, and small bronchioli while imaging them at a voxel side length of $350 \mathrm{~nm}$ with the use of SRXTM (Figure 5.1a and 5.1b). We were able to verify the locations of the observed gold particles by TEM (Figure 5.1c).

Particles with a size of $200 \mathrm{~nm}$ - a size smaller than the resolution achievable at TOMCAT-were imaged using TEM after selecting a particular alveolus in three-dimensional visualizations obtained from SRXTM data. Thirty minutes after instillation, particles were observed in cells-mainly macrophages-as well as in airspace. Most of the particles located in the airspace were in close contact to protein precipitations (Data not shown).

\section{$5 \cdot 5$ DISCUSSION}

A very high correlation between the two imaging modalities was observed. The virtual slices obtained from the SRXTM image stack (Figure 5.1b) and the real slices obtained using TEM (Figure 5.1c) have only been corrected for rotation and magnification. The correct vertical position of the serial TEM section in the sample was obtained through rigorous alignment and precise positioning of the sample in the microtome. 


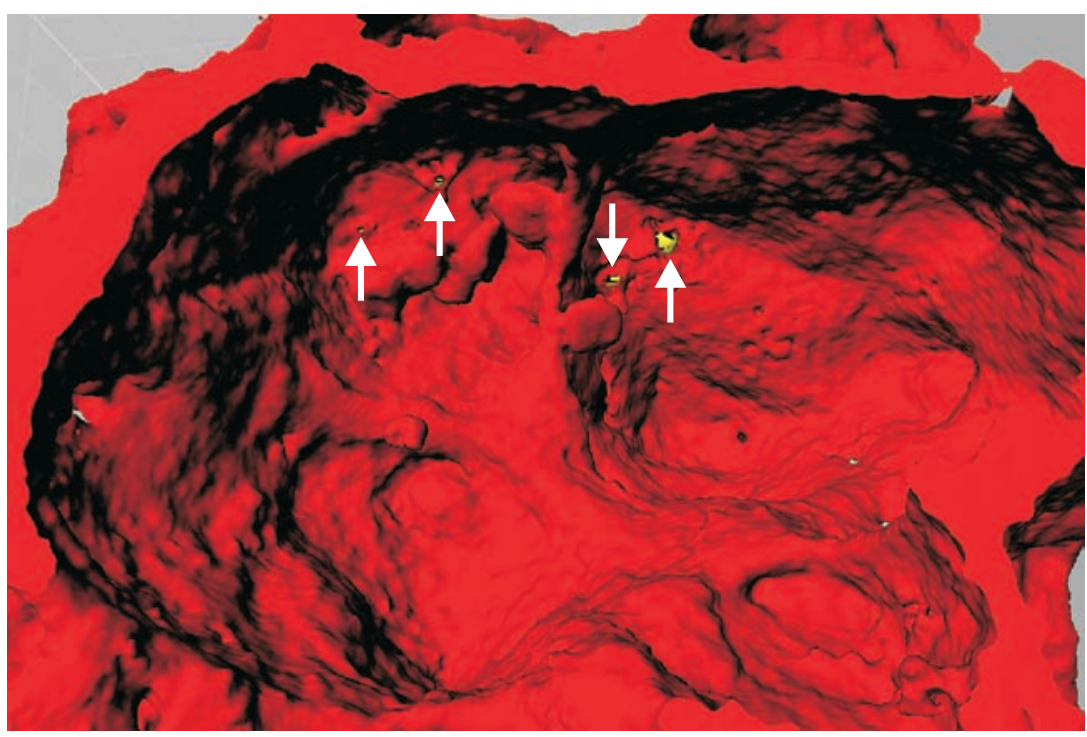

(a)

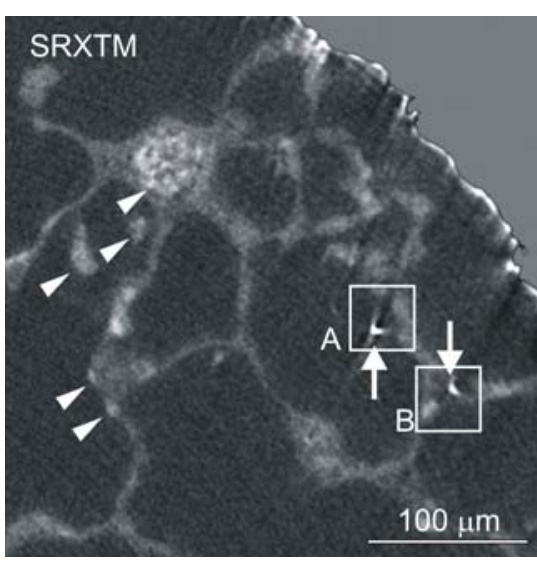

(b)

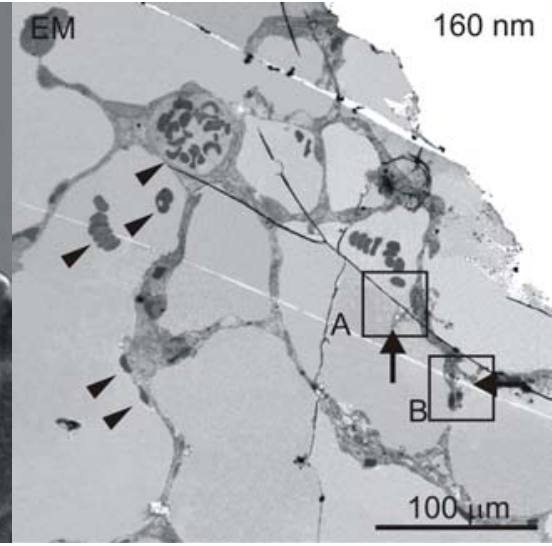

(c)

Figure 5.1: (a): Three-dimensional isosurface visualization of gold particles in the lung obtained from SRXTM data. Gold grains (yellow) are deposited in terminal airspaces. The air-tissue interface was removed on top of the gold grains during the visualization process in order to show the gold particles (arrows). (b): Detailed view of virtual SRXTM section obtained at TOMCAT containing two gold particles (arrows). The arrowheads are pointing to erythrocytes which are lighting up in the SRXTM images due to their high iron content of the hemoglobin. Due to the high contrast of gold the particles appear larger in the SRXTM images than they are in reality and we observed spiked image artifacts going out from the particles. (c): Corresponding TEM section of the virtual SRXTM section. The thin black lines in (c) represent folds in the serial section. The white lines in (c) represent knife marks from the sectioning process. 


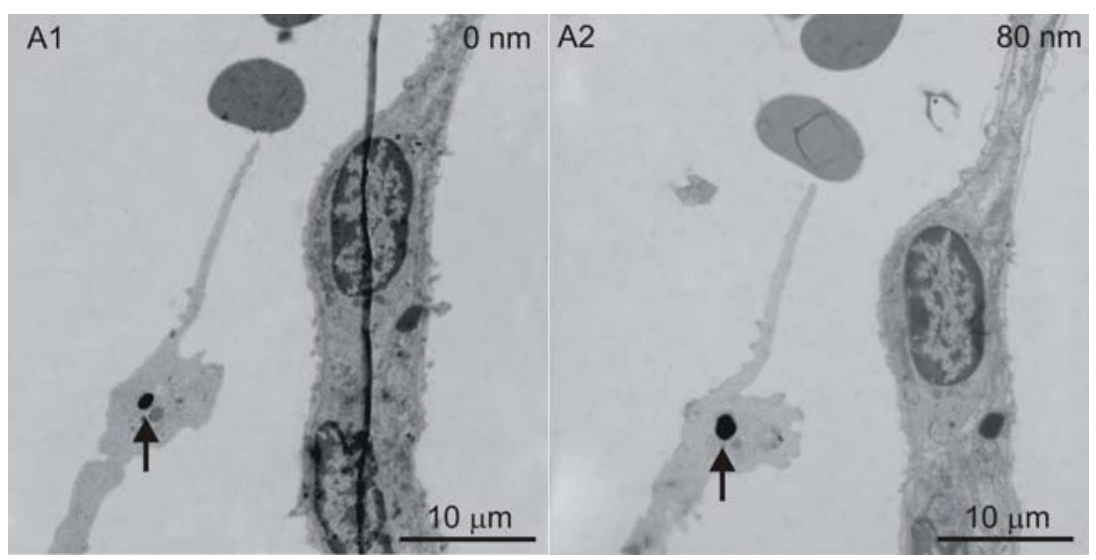

(a)

(b)

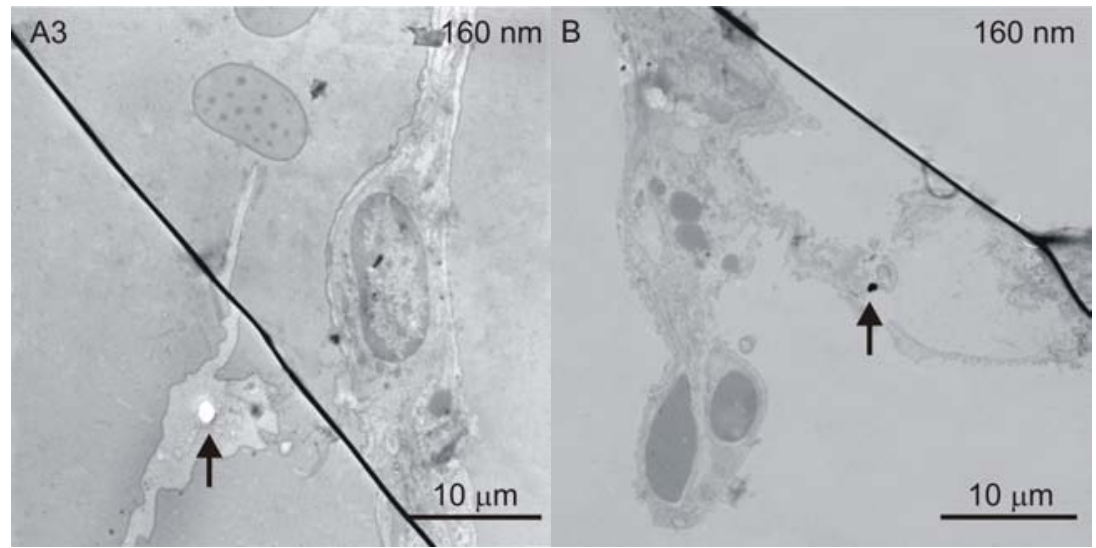

(c)

(d)

Figure 5.2: Transmission electron microscopy images of $700 \mathrm{~nm}$ gold particles: (a)-(c): Close-up view of square A of Figure 5.1b showing a gold particle (arrows) in consecutive TEM sections which are $80 \mathrm{~nm}$ apart. (d): Gold grain observed in square B in Figure 5.1b (arrow). Roughly half of the gold grains observed were located inside the cells, e.g. macrophages. The thin black lines visible in subfigures (a), (c) and (d) represent folds in the TEM section.

We have been able to track the gold particles over consecutive serial sections, even if they sometimes were not directly visible. As can be seen in Figure 5.2c, we sometimes only observed a hole where we would have expected to see the gold particle (arrow). Since the gold grains are much harder than Epon and do not stick well to the resin, it is expected that the grains will be pulled out of the Epon block as soon as half of the grain is cut.

\subsection{CONCLUSIONS}

We conclude that the combination of SRXTM and TEM allows the threedimensional localization of particles in the mammalian lung. In a synergistic way, we used SRXTM to obtain the full unrestricted 3D access and TEM to verify the localization of the particles in the $3 \mathrm{D}$-space with very high resolution. 
We are planning to use this method for the detection and localization of inhaled particles and as a mean of providing data for airflow simulation in the mammalian lung.

\section{$5 \cdot 7$ ACKNOWLEDGMENTS}

We thank Christoph Hintermüller and Federica Marone for expert help at TOMCAT and Bettina De Breuyn as well as Christoph Lehmann for the preparation of the samples. This work has been supported by Swiss National Science Foundation grant 3100Ao-109874 and by US National Heart, Lung, and Blood Institute grant HL-070542. 



\author{
Akira Tsuda ${ }^{1,2}$ \\ Nenad Filipovic ${ }^{3}$ \\ David Haberthür ${ }^{4}$ \\ Rene Dickie ${ }^{1}$ \\ Yasuto Matsui ${ }^{5}$ \\ Marco Stampanoni ${ }^{6}$ \\ Johannes C. Schittny 4
}

First published in: J Appl Physiol 105: 964-976, 2008.

doi:10.1152/japplphysiol.90546.2008

\title{
6.1 ABSTRACT
}

The alveolated structure of the pulmonary acinus plays a vital role in gas exchange function. Three-dimensional (3D) analysis of the parenchymal region is fundamental to understanding this structure-function relationship, but only a limited number of attempts have been conducted in the past because of technical limitations. In this study, we developed a new image processing methodology based on Finite Element (FE) analysis for accurate 3D structural reconstruction of the gas exchange regions of the lung. Stereologically well characterized rat lung samples [62] were imaged using high-resolution synchrotron radiationbased X-ray tomographic microscopy. A stack of 1024 images (each slice: $1024 \times 1024$ pixels) with resolution of $1.4 \mu \mathrm{m}^{3}$ per voxel were generated. For the development of FE algorithm, multiple regions of interest, containing 7.5 million voxels, were further extracted as a working subunit. 3D FEs were created overlaying the voxel map using a gridbased hexahedral algorithm. A proper threshold value for appropriate segmentation was iteratively determined to match the calculated volume density of tissue to the stereologically determined value [62]. The resulting 3D FEs are ready to be used for 3D structural analysis as well as for subsequent FE computational analyses like fluid dynamics and skeletonization.

I School of Public Health, Harvard University, Boston, Massachusetts, USA

2 Address for reprint requests and other correspondence: A. Tsuda, Molecular and Integrative Physiological Sciences, Harvard School of Public Health, 665 Huntington Ave., Boston, MA 02115 (e-mail: atsuda@hsph.harvard.edu).

3 University of Kragujevac, Kragujevac, Serbia

4 Institute of Anatomy, University of Bern, Bern, Switzerland

5 Graduate School of Engineering, University of Kyoto, Kyoto, Japan

6 Swiss Light Source, Paul Scherrer Institut, Villigen, Switzerland 


\subsection{INTRODUCTION}

The mammalian interthoracic respiratory tract can be divided into two areas: conducting airways and pulmonary parenchyma (i. e. , the gas exchange region of the lung). The conducting airways are a bifurcating network of relatively well-defined conduits carrying the ambient air to the parenchyma. Airways within the parenchyma-customarily called acinar/alveolar ducts - on the other hand, are not pipelike; they are formed by entrance rings of alveoli opening into a common passageway. Currently, enormous efforts (e.g. , [63-74]) are devoted to reconstructing the conducting airway network in three dimensions (3D), facilitated by rapid advancement of imaging technologies (e.g. , micro-Computed Tomography $(\mu \mathrm{CT})$, hyperpolarized-gas Magnetic Resonance Imaging (MRI) (HHe 3 MRI, etc.), as well as a rapid increases in computing power.

In contrast to conducting airways, lung parenchyma is harder to access; the structures are physically small and located distally in the respiratory tract. Basic structures of lung parenchyma are highly complex; this complexity can be easily envisioned by the fact that the enormously large surface area of lung parenchyma, as large as that of a tennis court in the case of human lungs [31, 75], for example, has to be folded in within the lung cavity. At the same time, the image resolution required to obtain reasonable details of this complexity is on the order of a few micrometers [considering, for instance, the thickness of alveolar septa (inter-air space septa) of roughly 10 $\mu \mathrm{m}$ ([75]; P. Gehr, personal communication)]. This required resolution is much finer than the resolution of commonly available MRI or even $\mu \mathrm{CT}$ mentioned above, but can be obtained with Synchrotron Radiation based X-ray Tomographic Microscopy (SRXTM).

Despite these difficulties, the motivations for reconstructing the $3 \mathrm{D}$ geometry of the respiratory region of the lung are numerous. Twodimensional (2D) sections of lung parenchyma are insufficient for many purposes and can produce erroneous models of lung structure [76]. For instance, 3D structural rendering is crucial to the study of micromechanics in 3D acinar microarchitecture, particularly given the likelihood of heterogeneous behavior with ventilation. Only by using a realistic 3D structure (for example, of alveolar shape and orientation of the alveolar opening with respect to the central thoroughfare acinar channel) can the qualitative and quantitative effects of 3D acinar geometry on gas and aerosol transport be determined. Despite the necessity of accurate 3D structural renderings of the gas exchange regions of the lung, there have been only a handful of attempts in the past (e. g. ., [7683]), mainly because formidable effort was required using traditional specimen preparation and imaging techniques.

Here we present our new effort at 3D reconstruction of the acinar air space in fixed lung tissue using the novel technology of SRXTM and subsequent 3D modeling of the obtained images. Because of the large specimen volume that can be imaged at high resolution using this method, we anticipate that it will ultimately allow $3 \mathrm{D}$ reconstruction of the entire air space of one acinus. Such a detailed rendering and consequent 3D quantitative analysis of structural measures of acinar morphology will prove invaluable to many studies, including performing the computational fluid dynamics of aerosol deposition in the gas exchanging areas of the lung. In this paper, we demonstrate the ability of 
SRXTM to render greater volumes $\left(10^{9}{\mu \mathrm{m}^{3}}^{3}\right)$ of lung tissue than is possible by confocal microscopy and to achieve greater resolution (voxel size $=1.4 \mu^{3}$ ) than possible by traditional $\mu \mathrm{CT}$. We describe state-ofthe-art Finite Element technology for $3 \mathrm{D}$ reconstruction of both tissue and air spaces in the acinus in detail; the outcome would be ready for subsequent FE computational analyses like fluid dynamics and skeletonization.

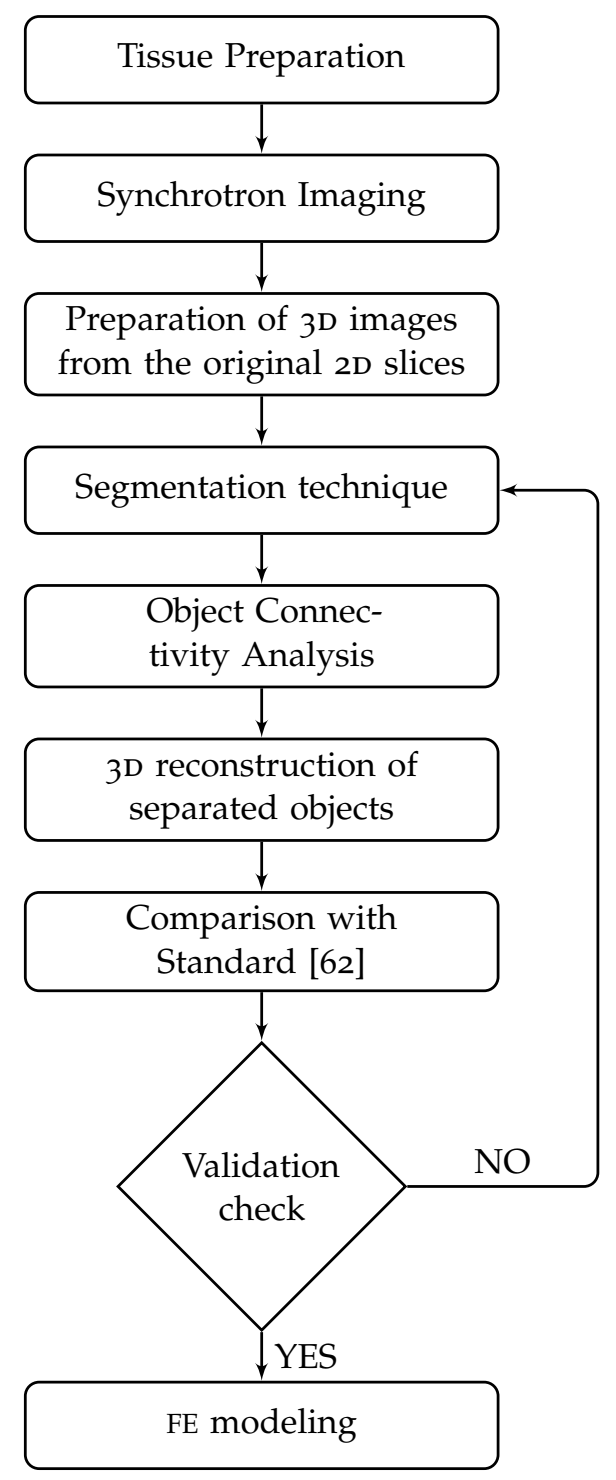

Figure 6.1: Workflow diagram outlining protocol for high-resolution 3dimensional (3D) reconstruction of the acinus.

\subsection{MATERIALS AND METHODS}

The experimental protocol for high-resolution 3D reconstruction of the rat acinus may be categorized into the following three components: tissue preparation, SRXTM, and image processing (Figure 6.1). The first two components have been previously described in detail elsewhere $[61,62,84,85]$ and thus are only recapitulated briefly here. The em- 
TOMCAT is a

beamline for

TOmographic

Microscopy and

Coherent rAdiology experimen $T$ s, which started regular user operation on June 2006. Located at the $\mathrm{X} 02 \mathrm{DA}$ port of the Swiss Light

Source (SLS), the beamline gets photons from a 2.9-T superbend. A Double

Crystal Multilayer

Monochromator

(DCMM) covers an energy range between 6 and $45 \mathrm{keV}$ with a bandwidth of a few percent down to $10^{-4}[61]$.

This particular energy enables us to achieve a high contrast between the tissue and the background of the sample.

Binning is the process of grouping together a certain number of input pixels to one output-pixel while recording the image; this is done to suppress the noise of the resulting image through pixel averaging and to reduce the file size. phasis of the present work is on the third component; the experimental methods are described in detail below.

\subsubsection{Animal Handling and Tissue Preparation}

Because our main aim in this study is development of new image processing methodology to reconstruct rat acinar structure in $3 \mathrm{D}$, we used existing glutaraldehyde-fixed lung tissue samples of 6o-day-old Sprague-Dawley rats (Zürich strain, [62]). The individual lung lobes were separated and their volumes were determined by fluid displacement [86]. Tschanz et al. [62] provide a comprehensive morphometric characterization of these fixed lungs; we use these data as a gold standard.

The left lung lobes were cut into $2 \times 2 \times 2 \mathrm{~mm}$ pieces, postfixed with $1 \%$ osmium tetroxide $\left(\mathrm{OsO}_{4}\right)$ in 0.1 m sodium cacodylate $(\mathrm{pH} 7.4$, 340 mmol per $\mathrm{kg} \mathrm{H}_{2} \mathrm{O}$ ), stained en bloc with $0.5 \%$ uranyl acetate $\left(\mathrm{C}_{4} \mathrm{H}_{6} \mathrm{O}_{6} \mathrm{U}\right)$ in $0.05 \mathrm{M}$ maleate buffer, dehydrated in a graded series of ethanol, and embedded in Epon 812. Note that fixation, dehydration, and embedding of the tissue likely contributed some shrinkage artifact. As in electron microscopy, osmium tetroxide and uranyl acetate were used for heavy metal staining of the tissue. Osmium tetroxide and uranyl acetate have a higher absorption than the unstained lung tissue. The higher contrast facilitates the imaging of the samples at TOmographic Microscopy and Coherent rAdiology experimenTs (TOMCAT) and subsequent image processing.

Handling of the animals before and during the experiments, as well as the experiments themselves, were approved and supervised by the Swiss Agency for the Environment, Forests and Landscape and the Veterinary Service of the Canton of Bern.

\subsubsection{SRXTM}

The Epon embedded samples were shaped down into rods of a diameter of $1.2 \mathrm{~mm}$ using a watchmaker's lathe. They were glued on a rodlike sample holder of a diameter of $3.0 \mathrm{~mm}$ using two-component epoxy resin-based glue (Araldite Rapid, Novartis, Basel, Switzerland). Special care was taken to ensure that the samples were mounted perpendicularly to the surface of the sample holder to fit exactly into the window of the camera (see Figure 6.2 for imaging setup).

\subsubsection{Image acquisition}

The samples were scanned at an X-ray wave-length of $1 \AA$ A (corresponding to an energy of $12.398 \mathrm{keV}$ ) at the beamline TOMCAT at the Swiss Light Source (SLS) of the Paul Scherrer Institut (Villigen, Switzerland) $[48,61]$. After penetration of the heavy metal stained sample, X-rays were converted into visible light by a thin Ce-doped YAG scintillator screen (Crismatec Saint-Gobain, Nemours, France). Projection images were further magnified by diffraction limited microscope optics and finally digitized by a high-resolution $2048 \times 2048$ pixel CCD camera. The $\times 10$ lens was used, exposure time set to $200 \mathrm{~ms}$, and $2 \times 2$ binning was selected to improve the signal-to-noise ratio, resulting in isotropic voxels of $1.4 \mu^{3}$ for the reconstructed images. 
To achieve a tomographic image for each sample, 1500 projections were obtained over a sample rotation of $180^{\circ}$. The projections were then reconstructed on a 16-node server farm (Pentium 4, $2.8 \mathrm{GHz}$ processor, 512 MB RAM) using an optimized filtered back projection algorithm. This reconstruction results in an image stack of 1024 image slices in tif-format (a total size of $2 \mathrm{~GB}$ ). This image stack is later on referred to as the raw images.

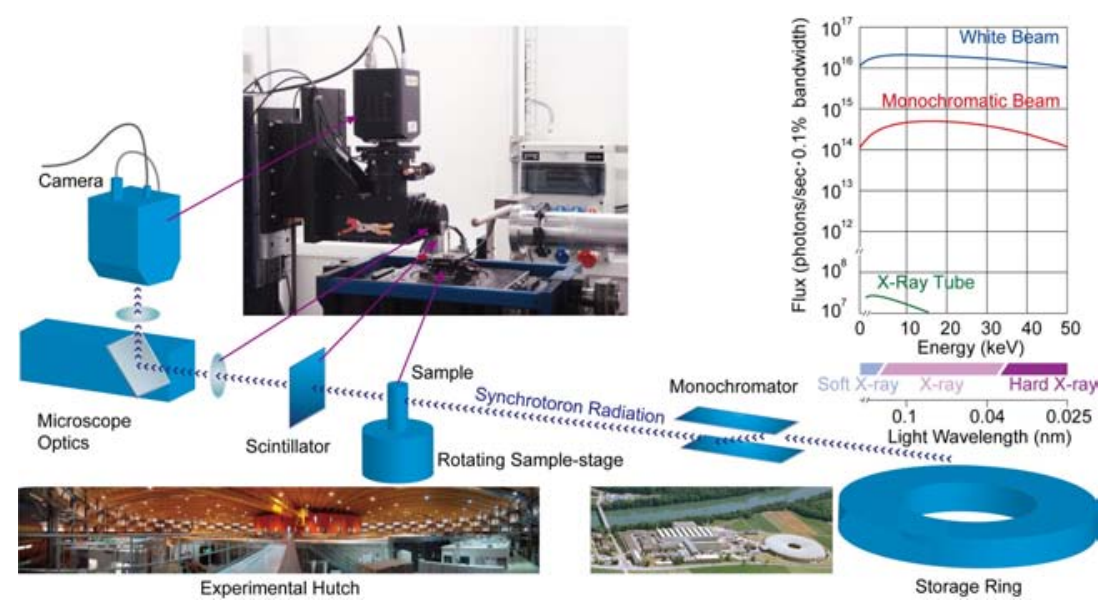

Figure 6.2: Imaging setup. The X-rays are coupled out of the storage ring via a wiggler. The monochromator is then used to select a desired wavelength of the incident $X$-rays. The sample is placed on a rotating sample holder inside the beam. While the $\mathrm{X}$-rays penetrate the sample, the stage is rotated, and in total 1500 projections over $180^{\circ}$ are recorded. Plot shows spectrum of TOMCAT (red and blue) compared with conventional X-ray tube (green).

\subsubsection{Image manipulation}

To facilitate detailed analysis for the development of 3D acinar reconstruction image processing, a cubic subregion of varying size was selected from the full dataset using the software Imaris (Bitplane, Zürich, Switzerland) installed on an Athlon 643500 based personal computer. Different subregions were chosen, typically representing a particular tissue structure, such as a cluster of alveoli without any large airways/branches, as well as a region without prominent ring artifacts. Ring artifacts represent an intrinsic problem of tomographic images and in our case are caused by very small particles trapped on the scintillator. They are usually limited to only some sections of the stack of images and are more prominent in the center of the sample. Before proceeding with the next step, we reduced the noise in some of the original $2 \mathrm{D}$ images and softened the image edges by using a Gaussian filter and/or a median filter with a $1 \times 3 \times 3$ kernel.

\subsubsection{Segmentation: thresholding and binarization}

To render our samples in three dimensions, we first segmented the raw data. Segmentation is the process of classifying the voxels of an image. Each voxel must represent either air space or tissue, thus, all the different gray values of the individual voxels are binarized into either o (air space) or 1 (lung tissue). Guided by the histogram of the gray scale values of the raw image (not shown), we selected an initial, 
tentative threshold value. Because the final selection of the threshold level is one of the most crucial steps in segmentation, special care has been taken to determine a correct threshold.

As indicated in Figure 6.1, we determined the appropriate threshold level iteratively:

1. we set an initial threshold level and performed segmentation;

2. we sequentially performed the object-connectivity analysis and 3D reconstruction of objects (described below);

3. we calculated and compared morphological parameters, such as volume density of tissue and surface area density of air space to those given by Tschanz et al. [62]; and

4. we readjusted/reset the threshold level based on the results of step 3 if necessary.

We performed this iterative process until the calculated morphological parameters closely matched the values of the gold standard [62].

\subsubsection{Object connectivity analysis}

Depending on the threshold level selected, erroneous holes and isolated small areas/objects may appear in the segmented images. To avoid these artifacts and to maintain object connectivity, we needed to preprocess the segmented voxels. We first determined the number of isolated objects and their sizes in the Region of Interest (ROI) and then eliminated the objects below a reasonable size $(\sim 5$ pixels $)$ by reclassifying voxel values (from airway to tissue, or vice versa). This Object Connectivity Analysis (OCA) was performed by implementing label adjacent pixel analysis in 3D [87-89]. For the sake of simplicity and clarity, we will explain this algorithm in 2D as follows.

Let us assume that black and white pixels (voxels in a 3D case) are distributed as in the example shown in Figure 6.3a. Scanning pixels from top to bottom and from left to right, we will examine the connectivity of each black pixel, by checking the colors (black or white) of its four adjacent pixels [a pixel on its left and three pixels on its top (top left, top middle, top right)]. We will assign a tentative Object Label Number (OLN) (consecutively, 1, 2, 3, 4...) to each black pixel and examine connections between identified objects. Four simple logical algorithms are involved.

1. If all four adjacent pixels are white, assign a new OLN to the current black pixel. [For instance, we assign $\mathrm{OLN}=1$ to the pixel at (column 2; row 2); OLN=2 to the pixel at $(9 ; 3)$, etc. (Figure 6.3b).]

2. If only one of the adjacent pixels has already been assigned an OLN, assign the same OLN to the current black pixel. [For instance, we assign the $\mathrm{OLN}=3$ to the pixel at $(4 ; 4)$ because the adjacent pixel on the left of it (at 3;4) has already been assigned with $\mathrm{OLN}=3$ (Figure 6.3b).]

3. If more than one adjacent pixels have already been assigned an OLN and if those adjacent pixels have the same OLN, assign the same OLN to the current pixel. [For instance, we assign OLN=4 to the pixel at $(6 ; 6)$ because the adjacent pixels at $(5 ; 6)$ and at $(6 ; 5)$ have already been assigned with an OLN=4 (Figure 6.3b).] 


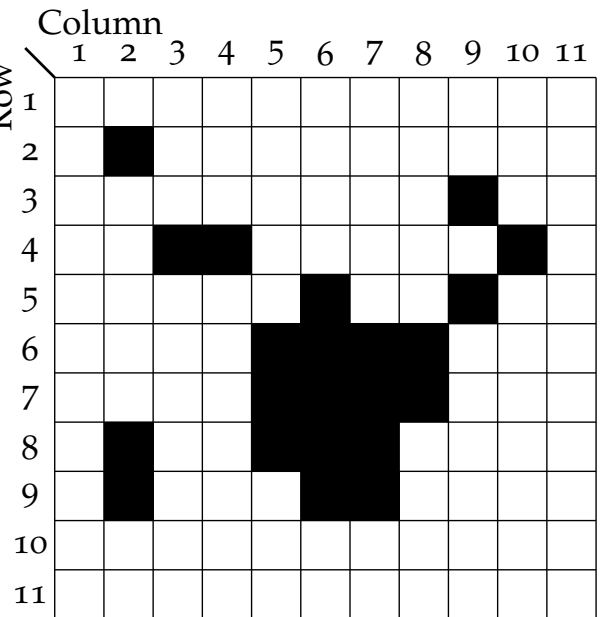

(a)

Column

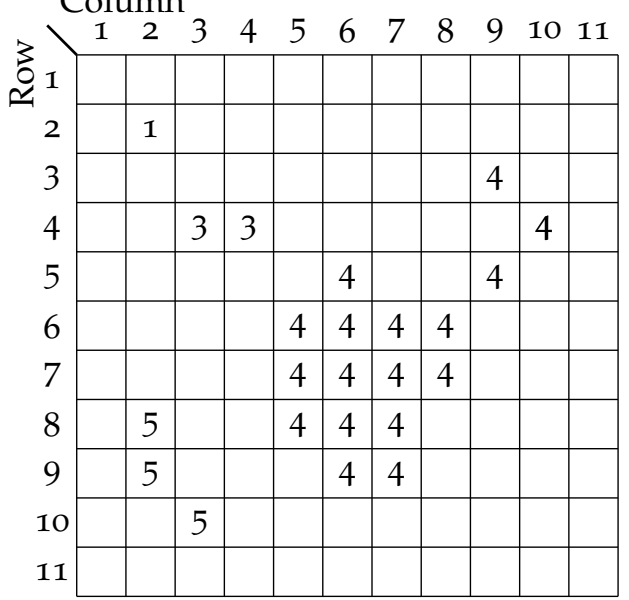

(c)

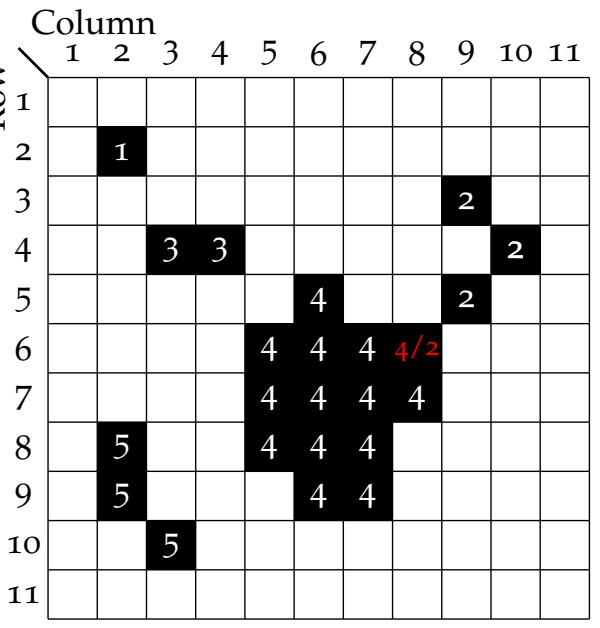

(b)

\section{Column}

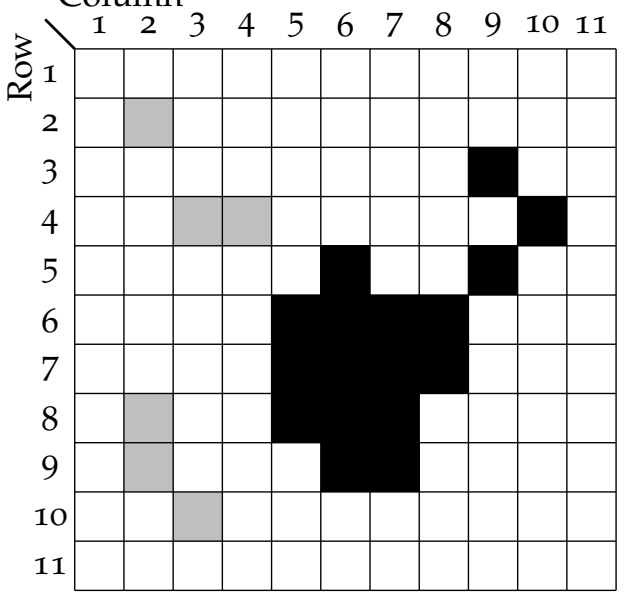

(d)

Figure 6.3: Object connectivity analysis shown in a 2-dimensional (2D) example. (a): a binary distribution of black vs. white pixels at certain threshold level; (b): An object label number (OLN) is assigned for each black pixel on the basis of the color (black or white) of four adjacent pixels; (c): on the basis of a flag pixel (red text in panel (b)), connectivity is identified between $\mathrm{OLN}=2$ and $\mathrm{OLN}=4$; $(\mathrm{d})$ : small objects less than cutoff ( $=4$ pixels in this example, light gray) are removed. 
4. If more than one adjacent pixel has already been assigned an OLN but if those adjacent pixels have different OLNs, assign one of the adjacent pixel's OLN and put a special marker (flag) on that pixel. The pixel with this flag will be re-examined in the next step. [For instance, we assign either OLN $=2$ or 4 to the pixel at $(8 ; 6)$ because the adjacent pixels at $(9 ; 5)$ and at $(7 ; 6)$ have already been assigned with OLN $=2$ and 4 , respectively. Thus, the pixel is marked with a flag (shown red in Figure 6.3b).]

After assigning OLNs to all the black pixels, we then re-examine the flagged pixels, which indicate that two or more appeared-to-be separated objects are indeed connected. We reassign the same OLN to all of the connected pixels leaving from the flagged pixel. For instance, we assigned $\mathrm{OLN}=4$ to the pixel at $(8 ; 6)$. This means that the pixels $(9 ; 3)$, $(10 ; 4)$ and $(9 ; 5)$ are also assigned with the OLN=4 (Figure 6.3c). Finally, we remove small objects that are physically unreasonable in size $(<5$ pixels; Figure 6.3d), suppressing noise and artifacts from the image acquisition.

\subsubsection{3D reconstruction of separated objects}

Much of the currently available medical imaging software for 3D reconstruction treats objects (i.e. , lung tissues in our case) as surfaces, and this is most often done by a triangulation of the voxel surface. However, to perform a complete 3D reconstruction, we use a different approach here; we reconstruct not only the surface of the boundary between the tissue and the air space, but also the volumes of tissues and air space by fitting FE to the raw voxel data and hence inside the full volume. For this, we use a Grid-Based Hexahedral Algorithm (GBHA) [90], which is explained as follows.

Let us consider an example in which we want to apply a threshold of 123 for the segmentation of our images. In Figure 6.4a, at left the thresholded voxels in a $3 \mathrm{D}$ representation is shown and, for the sake of discussion and clarity, a 2D representation is shown at right.

1. MESH GENERATION A FE mesh (Figure 6.4b), initially uniform, is isotropically generated over all pixels. We position the nodes of the FE mesh at the centers of the existing voxels (Figure 6.4b, right). This means that each FE overlaps $2 \times 2$ voxels in the $2 \mathrm{D}$ case. (Figure $6.4 \mathrm{~b}$, right) or $2 \times 2 \times 2$ voxels in the $3 \mathrm{D}$ case (Figure $6.4 \mathrm{~b}$, left). The black circles represent nodes generated inside the object and the white circles denote nodes outside of the object. The nodes inside the object have a pixel or voxel value higher than the chosen threshold and the nodes outside the object have a value lower than the chosen threshold. Each FE node is assigned the grayscale value of the corresponding voxel. Note that the boundary between the black and white nodes is not smooth at this stage.

2. SURFACE SMOOTHING I Because the FE mesh is located on the surface boundary, some of its nodes (shown as the white circles) are on the outside of the object. Additionally, the grayscale pixel values of those white nodes are lower than the chosen threshold value. By using a simple linear interpolation, we move these white nodes in the direction of the surface boundary toward the locations where the grayscale pixel value would exactly match 
the threshold value. There are multiple methods available to move the nodes by linear interpolation. We adapted the method developed by Schneiders [90] for our 3D case. It is important to note that in some cases, this linear interpolation might even move the node inside the object (see Figure 6.4c for examples).

3. SURFACE SMOOTHING II The translation of the nodes as described in step 2 may in some cases lead to a distorted (concave) FE surface. The distortion of the FE nodes can be evaluated with their Jacobian value. The Jacobian value is a matrix of the derivation of global to local FE interpolation function and the quality of any mesh can be directly evaluated by its Jacobian value [91]. Distorted FEs, which are not suitable for subsequent numerical calculations, show a negative Jacobian. To optimize the Jacobian, we implemented the standard Laplacian Smoothing Technique (LST) [92]. The LST usually takes a few loops (repetitions of step 2) over all FEs to achieve positive Jacobian values for all FEs. The results of applying the LST for 3D and 2D cases are shown, respectively, in Figure 6.4d, right ("After LST").

\subsubsection{Validation of Segmentation and Image Processing}

Once 3D reconstruction was performed with a certain tentative threshold value, the resulting 3D structure was evaluated against the gold standard, i. e. , well-characterized morphometric data provided by Tschanz et al. [62]. We calculated the volume density of tissue $\left(\mathrm{V}_{\mathrm{VS}}\right)$ and surface area density of air space $\left(\mathrm{S}_{\mathrm{VS}}\left[\mathrm{cm}^{2} / \mathrm{cm}^{3}\right]\right)$ to determine if there was unacceptable discrepancy. If so, we readjusted the threshold level and repeated the segmentation process, mesh generation, and subsequent image analysis until our calculated morphological parameters were within $<5 \%$ difference of the gold standard (Figure 6.1).

\subsubsection{Visualization of the 3D Reconstruction}

Once the calculated morphological parameters met the gold standard, the resulting 3D reconstruction was transformed in a standard VRML format for visualization as well as a proprietary DAT format for subsequent $\mathrm{FE}$ analysis.

\subsection{RESULTS}

The Epon embedded lung tissue samples of a 6o-day-old rat were selected from the lung tissue library of Peter Burri's lab (University of Bern). All morphological parameters of this particular rat, against which our data were compared, are available and published in Tschanz et al. [62].

\subsubsection{A Raw Image Stack and a Cubic Subregion}

The samples were scanned with SRXTM and a stack of 1024 raw images was reconstructed in tiff format (Figure 6.5a: stack of images, 6.5b: one slice of the raw data). The resolution of the image was $1.4 \mu \mathrm{m}$ per pixel, 


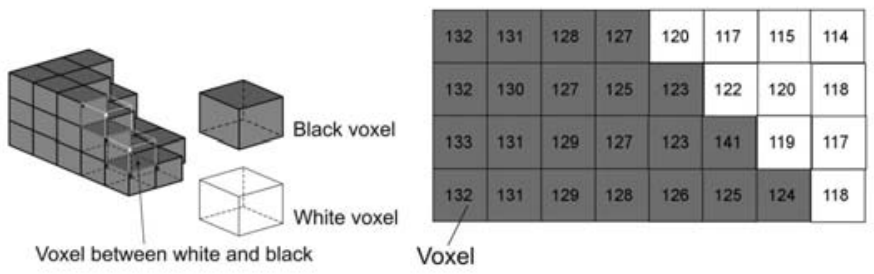

(a)
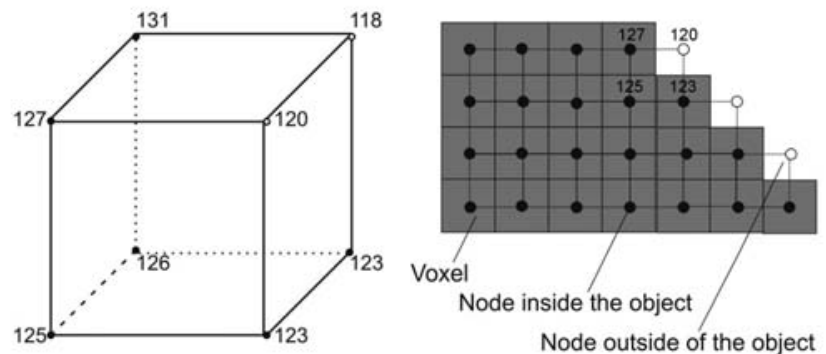

(b)
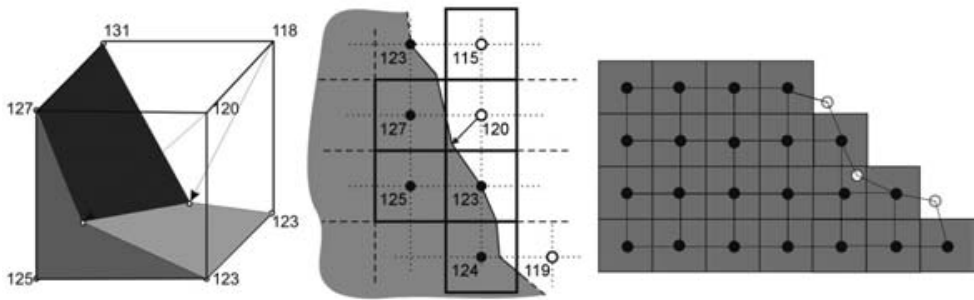

(c)
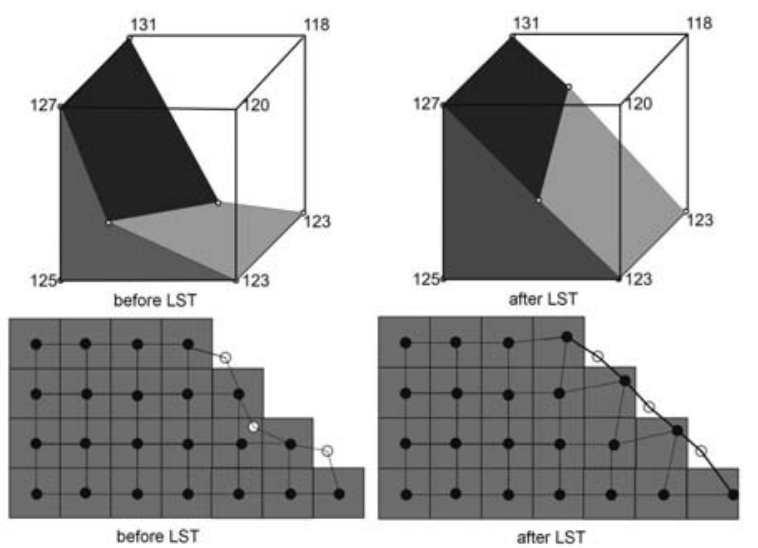

(d)

Figure 6.4: Grid-based hexahedral algorithm. (a): 3D thresholded voxels (left) and 2D representation of thresholded voxels (right). The grayscale values are kept in the map of voxel. (b): step 1: generation of uniform Finite Element mesh over the voxel map. For simplicity, FE nodes are positioned at the center of voxel. 3D FE meshing (left) and 2D FE meshing (right) are shown. Each node is assigned with the grayscale value from the corresponding voxel. (c): step 2: moving nodes on the surface due to trilinear interpolation in 3D case (left) and bilinear interpolation in 2D case (right). The location of surface boundary is shown in the middle image for 2D case. A fast sub FEvoxel level algorithm is implemented to locate a surface boundary. White nodes are moving toward this surface boundary. (d): step 3: Laplacian Smoothing Technique (LST). 3D and 2D before LST (left), 3D and 2D after LST (right). 
thus the edge length of the cubic stack was $1.4336 \mathrm{~mm}$, containing approximately one billion isotropic voxels.

To facilitate further processing of the image, different subregions of interest were extracted from the original raw data (one exemplary ROI is the orange small cube in Figure 6.5a, which is shown enlarged in Figure 6.5c. This ROI has a size of $196 \times 196 \times 196$ pixels (i.e. , an edge length of $\sim 275 \mu \mathrm{m}$ ) and contains $\sim 7.5$ million voxels. This is a reasonable size of data that can easily be handled by a standard PC (Duo Core 2 Pentium $2.33 \mathrm{GHz}, 2$ GB RAM) for visualization and volume rendering. The aforementioned size is an intermediate ROI; depending on the need for visualization/volume rendering other sizes have been chosen To validate the segmentation (see below), we split a big ROI inside the sample in the full dataset vertically into five subregions leading to a ROI size of $200 \times 200 \times 180$ pixels.

\subsubsection{Segmentation}

As mentioned, the selection of a threshold level has significant affects on segmentation. To demonstrate this, we have segmented a small crop of the original data (Figure 6.6a) with three different threshold values. With a low threshold level, for instance 130, the image would possess too many black voxels and the septa in the lung appear to be too thick (Figure 6.6b). On the other hand, with a higher threshold level, for instance 148 , the image would possess too many white voxels and the lung tissue appears to be very thin, with multiple erroneous holes in the septal walls (Figure 6.6d). With an intermediate threshold, for instance 139, the lung structure appears to be reasonable, with a minor amount of holes (Figure 6.6c). The optimal value of the threshold level was iteratively determined by performing a readjustment-recalculation process to match some of the key morphological parameters in the resulting 3D reconstruction to the gold standard. This is explained below in detail.

\subsubsection{FE 3D reconstruction}

Prior to the 3D reconstruction, the was performed to screen small objects with unreasonable size; voxels of those unreasonably small objects were reclassified to match the surrounding voxels. After successful preprocessing, 3D reconstruction was performed using GBHA. The examples with three different values of threshold level (130, 139, 148), corresponding to those in Figure 6.6, are shown in Figure 6.7. As is expected, the 3D reconstructed object appears to have thick tissue walls with the lower threshold value of 130, while it appears to be too thin, with many holes with the higher threshold value of 148 . With the intermediate threshold value of 139 , the structure appears to be reasonable. This will be tested quantitatively next.

\subsubsection{Validation of Segmentation and Image Processing}

To perform a statistical analysis for the selection of the threshold value, five pieces of parenchymal sample $(200 \times 200 \times 180$ pixels $)$ were selected. We calculated the $V_{V S}$ of each ROI after the 3D reconstruction, and the average value with Standard Error (SE) was plotted as a function of 


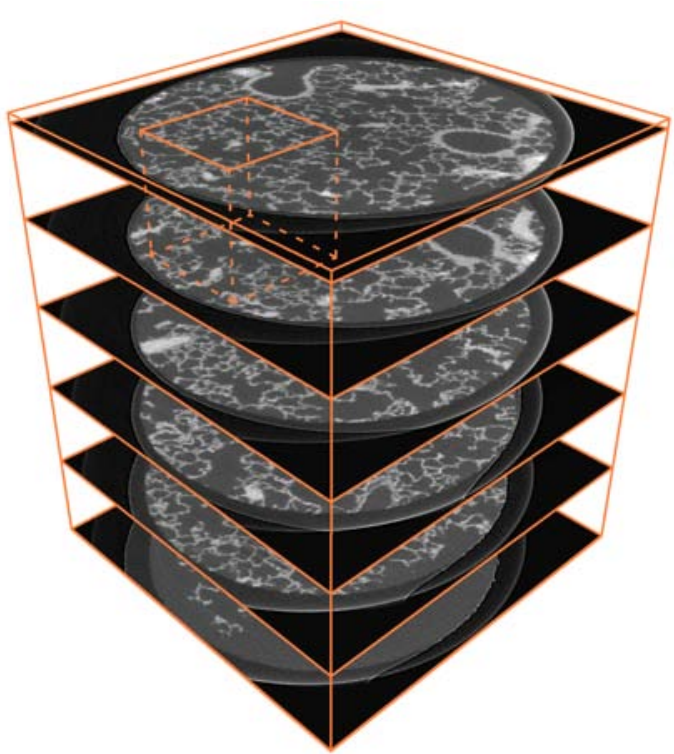

(a)

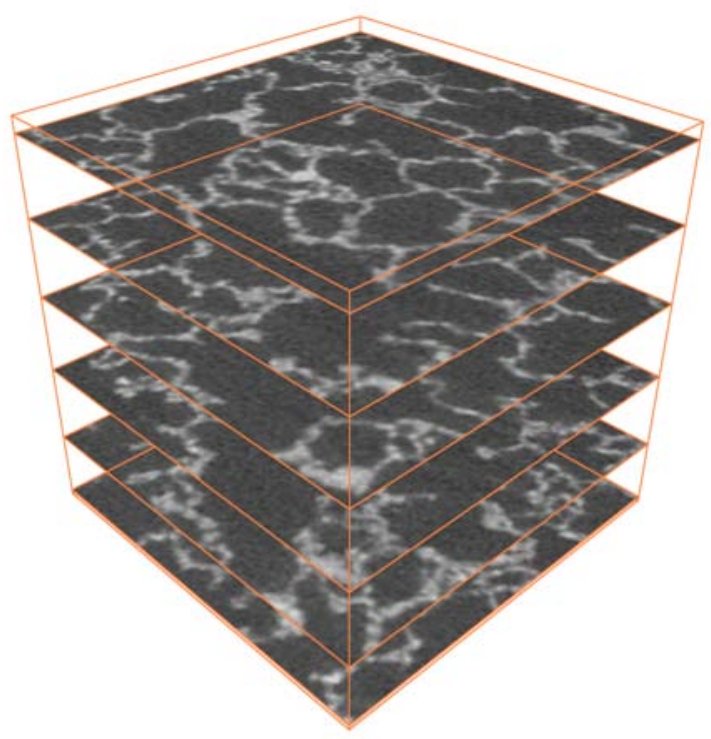

(c)

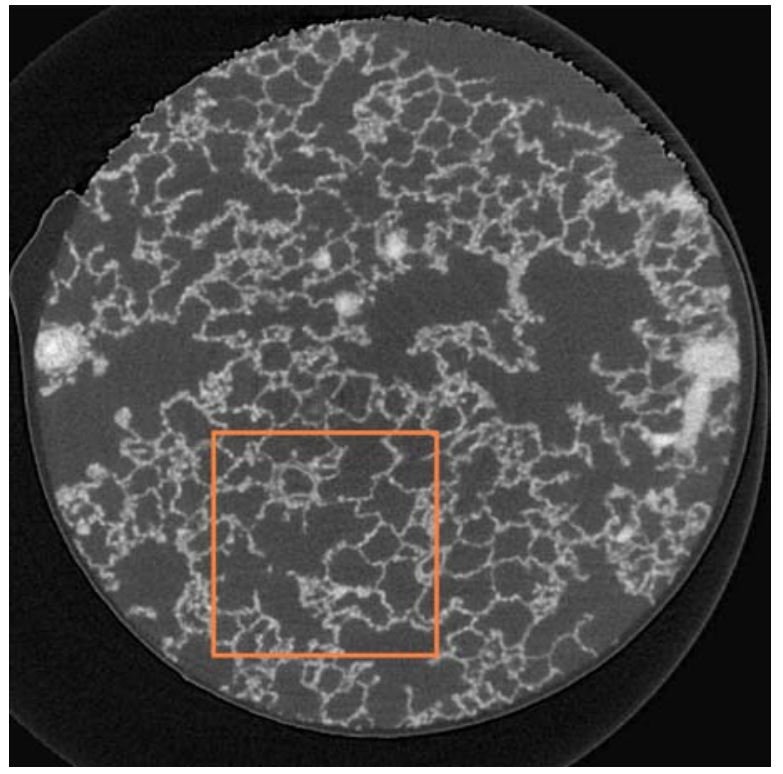

(b)

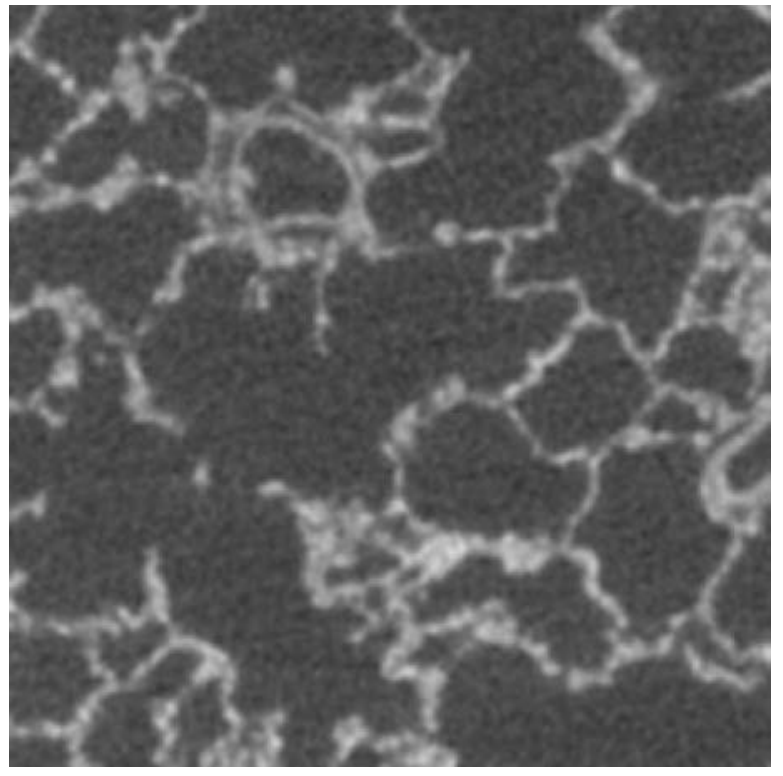

(d)

Figure 6.5: Overview of workflow for the selection of a Region of Interest (ROI). (a): full perspective view of the sample R108C6oC-o7e with 6 selected orthogonal slices and bounding box of the full sample in orange. The ROI selected for subfigures (c) and (d) is visible in the top left. (b): close-up of one slice of the full dataset with selected ROI (side-length of 256 pixels). (c): ROI cut out of the full sample, 6 selected orthogonal slices are shown. (d): close-up of the ROI in subfigure (b). 


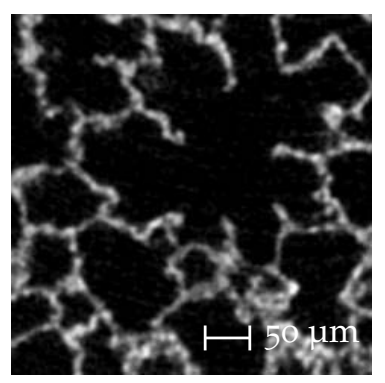

(a)

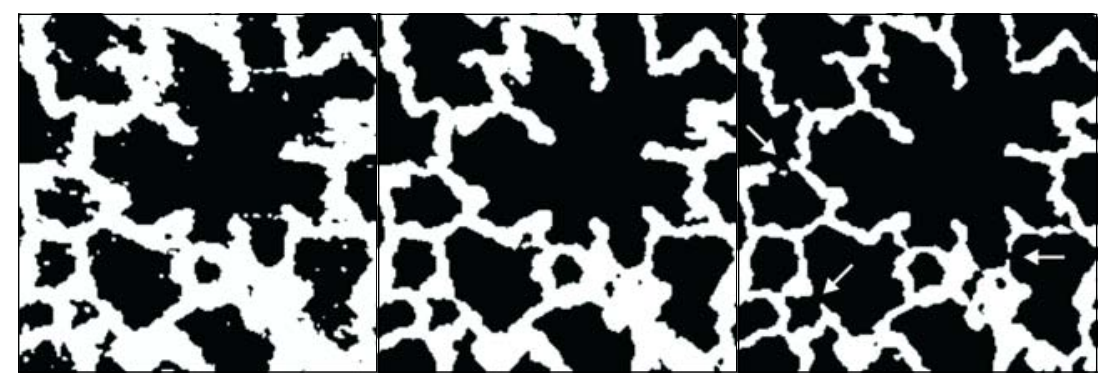

(b)

(c)

(d)

Figure 6.6: (a): original slice from raw data. (b): thresholded with a value of 130, meaning that all pixels below 130 are set to black, all pixels with a value of 130 or greater are set to white. Resulting alveolar walls are too thick. (c): thresholded with a value of 139. Image looks correct. At this threshold the observed thickness of the septa was smaller than $10 \mu \mathrm{m}$. In the same animals [93] the thickness of the septa was determined as they appeared on electron microscopical images. They measured a mean of $8 \mu \mathrm{m}$. (d): thresholded with a value of 148 . Alveolar walls are too thin, and multiple erroneous holes (arrows) appear in the tissue.

threshold level (Figure 6.8). Our calculated value of $\mathrm{V}_{\mathrm{VS}}$ nearly linearly decreased with increasing threshold level and our $V_{\mathrm{VS}}$ matches the gold standard value of 0.196 at a threshold value of 139 . In addition, the values of calculated SE were generally very small, indicating that each sample, although small in size, represented typical parenchymal structure. We determined to use a threshold value of 139 for this tissue sample.

The surface area density of air space $\left(\mathrm{S}_{\mathrm{VS}}\left[\mathrm{cm}^{2} / \mathrm{cm}^{3}\right]\right)$ of each ROI sample was also calculated and plotted (Figure 6.8). As we expected, however, $S_{\text {VS }}$ shows poor match with the reported value of $904 \pm 84$ (SE) over almost the entire range of threshold levels tested and especially at the threshold value of 139 . The cause of this discrepancy will be discussed in detail in section 6.5.

\subsubsection{FE 3D Reconstruction of Air Space}

3D air spaces, separated by tissues, in the ROI were also constructed (Figure 6.9). To demonstrate air space configuration more clearly, a small alveolated air section was isolated from the center of ROI and was enlarged in an inset of Figure 6.9. These 3D air objects packed with several million 3D 8-node FEs (a mixture of structured and unstructured 


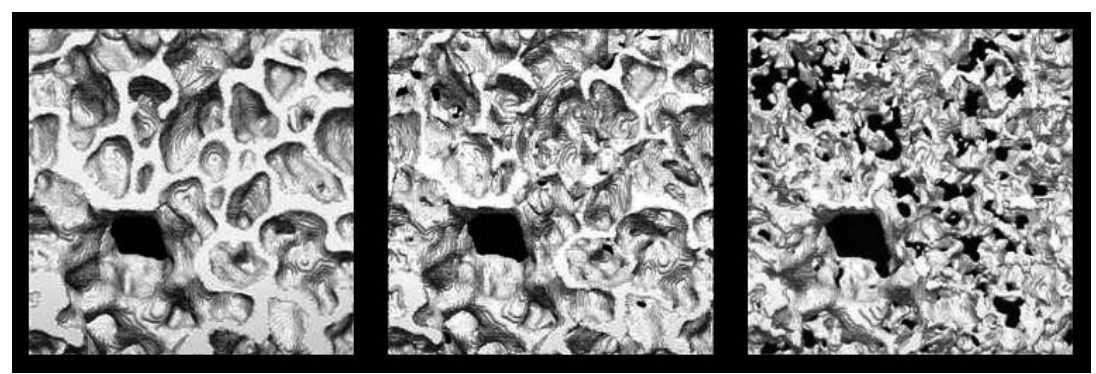

(a)

(b)

(c)

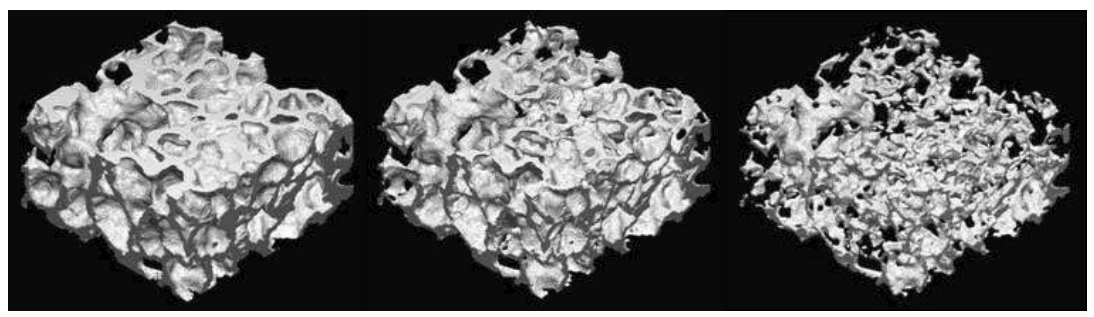

(d)

(e)

(f)

Figure 6.7: Three-dimensional reconstruction of tissue. (a) and (d): threshold 130. (b) and (e): threshold 139. (c) and (f): threshold 148.

cubic elements) can be readily used for FE calculations, including airflow Computational Fluid Dynamics (CFD), in the future.

\subsection{DIsCUSSION}

We described our custom-made FE methodology to reconstruct the acinar air space as well as septal tissue of rat lung in three dimensions. Approximately $3 \mathrm{~mm}^{3}\left(=3 \times 10^{9} \mu^{3}\right)$ of fixed lung tissue was scanned with high resolution SRXTM (voxel size $=1.4 \mu^{3}$ ) and a stack of 1024 image slices $(1024 \times 1024$ pixels) was prepared for $3 \mathrm{D}$ rendering. After preprocessing the raw voxel data with a tentative threshold level, 3D FEs were created overlaying the voxel map using a GBHA. A proper threshold value for adequate segmentation was iteratively determined to match the calculated volume density of tissue $\left(\mathrm{V}_{\mathrm{VS}}\right)$ to the stereological determined value [62]. With the optimized algorithms (a combination of OCA and GBHA algorithms), CPU time for one iteration for $\sim 8$ million voxels was $\sim 90$ min by a standard PC.

\subsubsection{Historical Perspective}

Acinar structure was traditionally studied using lung cast models [9496] or serial histological sections (e. g. , [77, 81, 97-99]). With the former approach, some types of measures are not possible: only the polymerfilled air spaces are available for measurement as the tissue is digested away, and internal data are lost as scanning electron microscopy is limited to the exposed surfaces of the cast. 3D rendering of alveolar geometries from histological sections requires careful, time-consuming alignment and registration of serial $2 \mathrm{D}$ sections [80, 82], which generally limits reconstruction to just a few alveoli [80]. An additional 


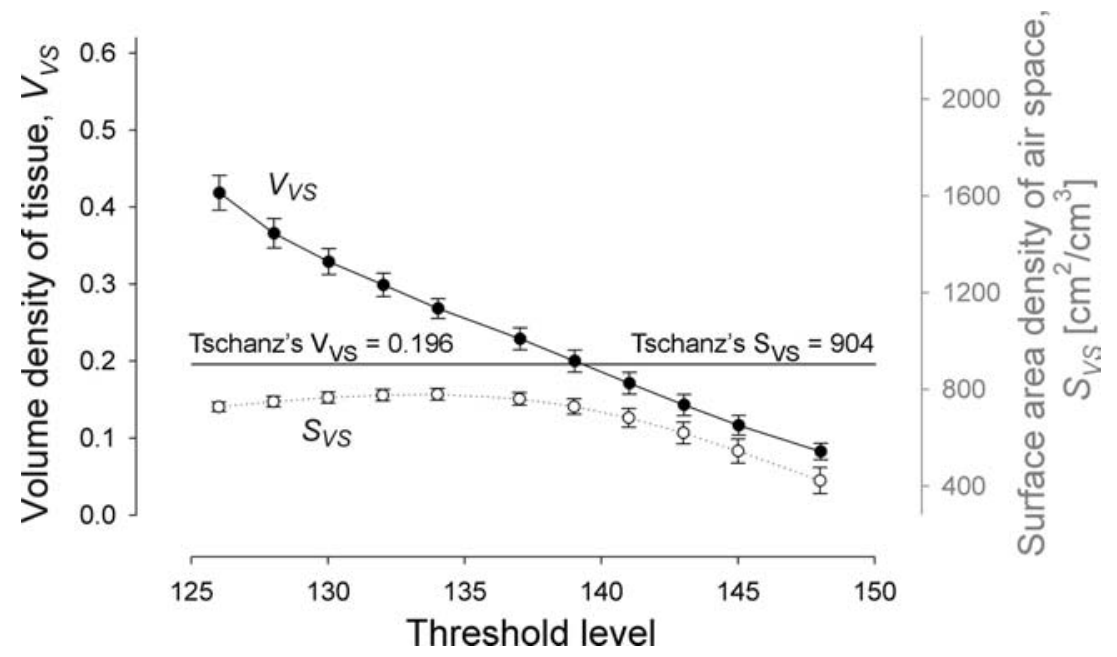

Figure 6.8: Average volume density of tissue $\left(\mathrm{V}_{\mathrm{VS}}\right)$ and surface area density of air space $\left(\mathrm{S}_{\mathrm{VS}}\right)$ of ROIs, plotted as a function of threshold level. Error bars represent standard error. Our $\mathrm{V}_{\mathrm{VS}}$ matches the gold standard value of 0.196 at a threshold value of 139. Bars include the standard error, $\mathrm{n}=5$.

drawback is sample destruction and deformation from physical sectioning.

Optical sectioning permits faster, less invasive, more thorough digital analysis and 3D rendering than is possible with physical sectioning. Large upper airways can be analyzed with modern techniques such as magnetic resonance microscopy [100, 101] or optical coherence tomography [102]; functional imaging of the lung by Computed Tomography (CT) and MRI has recently been summarized by van Beek and Hoffman [103]. Laser scanning confocal microscopy provides sufficient resolution to image fine-scale acinar detail, but there have been very few 3D acinar reconstruction studies using this method. Cookson et al. [76] used serial optical sections acquired by confocal microscopy to produce 3D volume renderings of human alveolar ducts. This approach is restricted in that imaging depth below the tissue surface is limited by light penetration and the working distance of the objective lens [104]. Cookson et al. [76] warned that caution was necessary in interpreting confocal 3D renderings because the relative contributions of the various factors (refractive index changes, tissue density changes, resorption) causing depth-dependent loss of resolution and/or intensity were difficult to measure and correct. Multiphoton microscopy improves imaging depth, but the imaging depth is still restricted to $\sim 100 \mu \mathrm{m}$ into the lung [105]. For comparison, human alveolar ducts range from 270 to $600 \mu \mathrm{m}$ in diameter [106]. Thus sample size constraints limit confocal analysis to just a small portion of the acinus, restricting its usefulness in studies of flow and aerosol particle deposition. In the early 1990s, new imaging techniques, such as СТ [107, 108], permitted measurement and reconstruction of the 3D structure of the bronchial tree and small airways [63, 72, 109-114]. Successful visualization of the acinar region, though, was limited by the spatial resolution of standard $\mu \mathrm{CT}$. In the past few years, however, $\mu \mathrm{CT}$-based 3D reconstruction and morphometric analysis of the alveolar region has become possible [79, 83, 115], facilitated by the advent of Synchrotron Radiation Computed Tomography (SRCT). SRCT is to be distinguished from 
SRXTM $[18,48,61]$. While during SRCT the X-rays are directly recorded after transmitting the sample, during SRXTM the transmitted images are first recorded on a scintillator and magnified 5-20 times by a light microscope before recording.

\subsubsection{SRXTM}

Synchrotron Radiation (SR) is an electromagnetic wave emitted from electrons traveling near the speed of light when their path is bent by a magnetic field [116]. A synchrotron facility is an excellent source of the most useful electromagnetic waves to explore materials and biological systems: X-ray ultraviolet and infrared light. Here we limit our discussion particularly to the application of SR for X-ray tomographic microscopy (Figure 6.2) highlighting the following superb features.

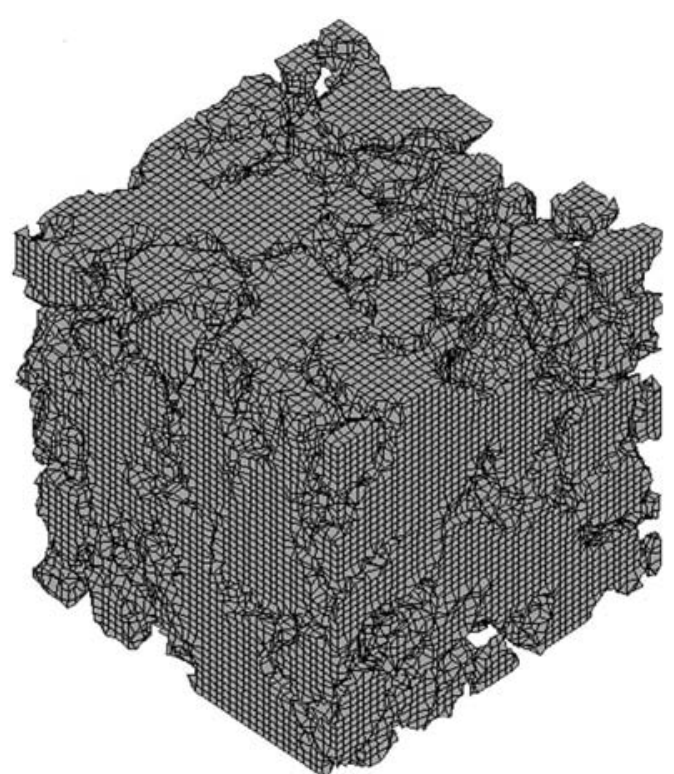

(a)

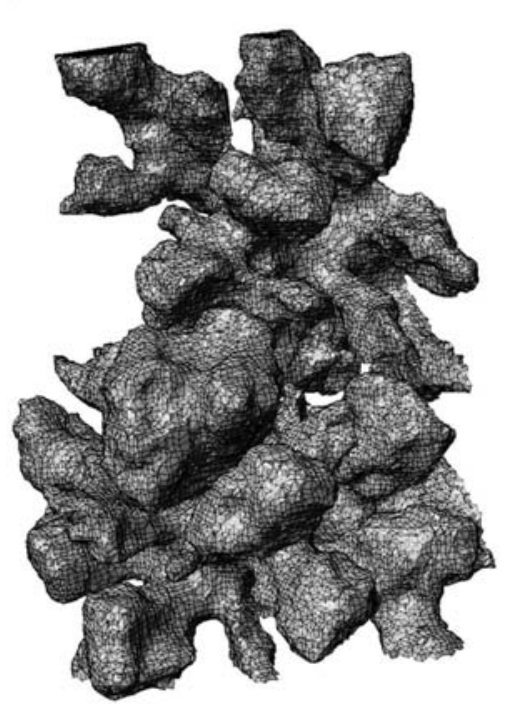

(b)

Figure 6.9: (a): reconstruction of 3D air spaces separated by tissues from ROI. (b): enlargement of a small alveolated air section isolated from the center of the ROI.

A monochromator is a device in X-ray optics that is used to select a defined wavelength of the radiation for further use in a dedicated instrument or beamline.

\subsubsection{High power and high brilliance}

SR is extremely powerful but even more important is that this radiation is emitted by a small source area and that this emission occurs within a narrow angular cone. Bending magnets or sophisticated insertion devices (called undulators or wigglers) produces therefore a high brilliant radiation that is several orders of magnitude more bright than conventional $X$-ray tubes (Figure 6.2). This makes the usage of monochromators particularly interesting since an extremely intense photon flux at a selected energy (or wavelength) can easily be extracted from the white beam emitted by the source (Figure 6.2). The X-ray energy can be adjusted ad hoc to the sample, according to its absorption properties. The monochromaticity of the beam is an important feature of SR application, critical for tomography applications since it auto- 
matically eliminates beam hardening artifacts from the tomographic reconstruction.

The high collimation of the SR produces de facto a parallel beam at the sample location: the resulting negligible geometrical blur makes it possible to obtain images with high spatial resolution and a high Signal-to-Noise Ratio (SNR). Since, for a given SNR and a given contrast, the necessary photon flux scales with the fourth power of the spatial resolution [104] it is obvious that SR-based tomography is perfectly suited for investigations with spatial resolution in the micrometer or even submicrometer range. Nowadays, $X$-ray tomographic microscopy endstations installed at third generation synchrotron facilities like TOMCAT [61] routinely reach resolution $\sim 1 \mu \mathrm{m}$ within scan times of a few minutes.

Synchrotron radiation-based СT overcomes the critical technical issue (resolution) that has prevented fine-scale structural analysis of the acinus by tabletop X-ray $\mu \mathrm{CT}$. The brilliant, highly collimated beam provides a nearly ideal X-ray source for $\mu \mathrm{CT}$ [117], producing greater spatial resolution. This allows high-resolution imaging of unprocessed lung tissue $[15,118-121]$ and ultra high resolution (from $<1$ to $15 \mu \mathrm{m}$, with tissue thicknesses of $\geqslant 1500 \mu \mathrm{m}$ ) visualization and $3 \mathrm{D}$ reconstruction of small airways and alveoli in processed tissue [18, 122].

\subsubsection{Segmentation/Threshold}

Careful determination of the proper threshold level is essential for accurate $3 \mathrm{D}$ rendering and for the first step of FE-mesh generation. If the level is too low, the fraction of air would become unrealistically small relative to tissue resulting in thicker septa. If the level is too high, on the other hand, the fraction of air becomes unrealistically large relative to tissue; thinner tissue would result in artificial holes in the septal walls or even too many unconnected tissue pieces in the sample. This would have a direct impact on the skeletonization process (which is not dealt with in this paper), as well as the subsequent FE analyses, such as airflow simulations. Our OCA is designed to minimize this problem.

A unique aspect of our work is that segmentation was done iteratively by searching for the optimal threshold value (Figure 6.1). In every iteration, the key morphological parameter (discussed below in detail) was calculated and compared with the gold standard; the iteration was continued until the calculated value matched the gold standard. This gold standard was previously determined in the tissue sample from exactly the same animal [62]. Because our main aim in this work was to develop FE methodology, this selection of tissue sample, whose morphology has been thoroughly analyzed, is very well suited for this project.

The key morphological parameter we tried to match was the volume density of tissue $\left(\mathrm{V}_{\mathrm{VS}}\right)$. The $\mathrm{V}_{\mathrm{VS}}$ is an easy to calculate parameter (i. e. , the sum of FE volume belonging to tissue divided by the sum of all FE volume) and is relatively insensitive to the resolution of the measurement technique. The calculated $V_{V S}$ decreases almost linearly with an increase in the threshold level (i. e. , a fraction of air volume relative to tissue volume) around the gold standard value $\left(\mathrm{V}_{\mathrm{VS}}=0.196\right.$ in our case, see Figure 6.8). At the threshold value of 139, the calculated $V_{V S}$ was within $2 \%$ difference of the gold standard. 

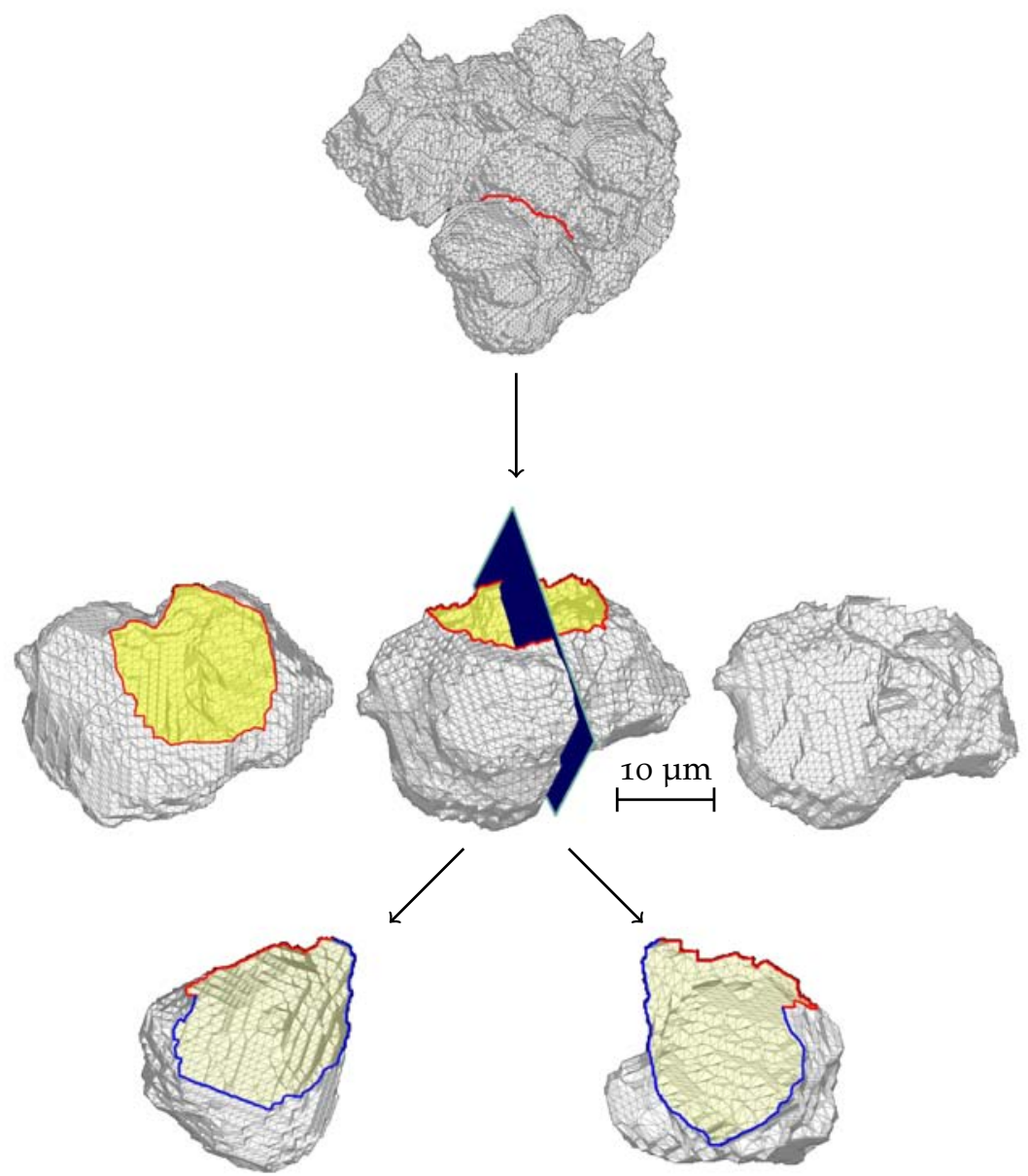

Figure 6.10: Three-dimensional FE shell model of acinus viewed from different angles. Top: ROI. Middle: top view (left); side view (middle); bottom view (right). Bottom: alveolar inside views.

We also calculated surface area density of air space $\left(\mathrm{S}_{\mathrm{VS}}\left[\mathrm{cm}^{2} / \mathrm{cm}^{3}\right]\right)$ and compared it with the value reported by [62]. However, our calculated values were always lower than the reported value $\left(\mathrm{S}_{\mathrm{VS}}=904\right.$ $\left.\left[\mathrm{cm}^{2} / \mathrm{cm}^{3}\right]\right)$. This is because although the volume is relatively insensitive to the resolution of the measurement technique, the air-tissue boundary (surface area) is highly sensitive to how it is measured. $\mathrm{S}_{\mathrm{VS}}$ reported in Tschanz et al. [62] was measured at a $\times 10$ secondary magnification after recording the electron microscopical images at a primary magnification of $\times 1200$ on a $35 \mathrm{~mm}$ film (theoretical resolution of $\sim 70 \mathrm{~nm}$ ), while the resolution of our $3 \mathrm{D}$ reconstruction is $1.4 \mu \mathrm{m}$. The extent of surface complexity is recognized differently, depending on the resolution of the measuring instrument; the finer the resolution of the sample and the instrument, the more surface detail can be detected. This is similar to the problem when one measures the length of the British coastline with different yardsticks [26].

6.5 .4 2D vS. 3D

Morphological analyses in 3D are qualitatively different from stereology, which is an analysis made in 2D sections to infer $3 \mathrm{D}$ structure 
and morphometry. Apart from the most common measures of geometric and topological properties, such as the volume density, the area density, and the length density, stereological analysis is generally limited. For example, number density cannot be estimated stereologically using single sections without a significant number of a priori assumptions about the shape of the objects, including the question of whether the objects are convex and whether they are simply connected. These significant restrictions in the stereological analysis are due to the fact that it is based on the following two fundamental assumptions:

1. the structure is homogeneously distributed (i.e., spatially invariant) and

2. isotropically distributed (i.e., rotation invariant) in the tissue sample (J. P. Butler, personal communication).

The 3D analysis relaxes these restrictions either by going to full unrestricted 3D ( $\mu \mathrm{CT}$, MRI, SRXTM, etc.) or using two consecutive sections (disector) for the counting of numbers [12].

In addition to these fundamental differences, 3D analyses have numerous advantages over traditional stereology. The most obvious advantage is the flexibility of the analysis. It is often difficult to properly select a ROI because the exact location of the target region cannot be identified beforehand and in the traditional approach, one has only one opportunity to section the tissue sample. In the case of $3 \mathrm{D}$ reconstruction analysis, on the other hand, the selection of the ROI can be repeated as many times as required and the selection can be iteratively improved until the target ROI is obtained. Once the ROI is selected in 3D (Figure 6.10, top), the target region (e.g. , an alveolus) can be viewed from any arbitrary angle (see Figure 6.10, middle), including from behind the object (Figure 6.10, middle right), something not easily achievable otherwise. The 3D object can be cut into half (Figure 6.10, bottom) and also be sliced to make 2D sections in any orientation with any slab thickness (data not shown) and there is no limitation in repeating the sectioning process. Furthermore, since the ROI can be viewed in any chosen angle and an internal view is possible, it is possible to fly through the structure to gain an internal view of the conduit once the 3D reconstructed structure is electronically available.

\subsubsection{FE 3D Rendering}

It is important to make a clear distinction between our FE 3D rendering approach and the traditional surface triangulation approach that is most common in medical imaging software. The triangulation approach is mainly used for surface visualization; this uses a variety of filtering methods and surface smoothing techniques, but does not involve real volume like with our FE 3D rendering method. If the volume compartment of either air or tissue is needed for subsequent analysis, such as for a calculation of fluid flow in acinar air space, a traditional surface triangulation approach does not provide a readily usable 3D structure; a volume bounded by the visualized surface needs to be further elementalized. Since we are in possession of a closed workflow for the further processing of the meshed surfaces (i.e. , there is a single process from mesh generation through to visualization), intermediate conversions of the dataset into other formats are not required. 
An STL file describes a raw unstructured triangulated surface by the unit normal and vertices of the triangles using a 3D Cartesian coordinate system.
This eliminates overhead and speeds the overall process. The FE mesh of the data makes it possible to use it for volume rendering, further processing (with the use of quasi-standard STL file format), and direct import into the software used for CFD calculations and evaluation of morphological factors. Since we also obtain a mesh inside the structure, we lay the groundwork for the skeletonization (extraction of the mean middle line) of the terminal airways and the determination of the entrance ring of a single alveolus.

The main aim of this project is a direct elementalization of air (or tissue) space using FE technique. By isolating air (or tissue) from the rest (tissue or air, respectively), the interface of these two volume components, i.e. , an air-tissue barrier, is automatically identified. For this isolation, we adapted the GBHA proposed by Schneiders [90]. First, an FE mesh was overlaid on the voxel map. Basically, a relation of one FE to one voxel could be used, but one practical problem may arise. That is, if the number of voxels in the original data is too large (i.e., in an order of billion in the case of our raw data) for a common PC to handle easily, we may need to reduce the number ratio between FE and voxel. The important points to achieve are, generally,

1. to reduce the number of FEs to a reasonable number but

2. to still produce a smooth surface boundary.

We use hexahedral FE for this. An air-tissue interface was smoothed by adjusting the locations of FE nodal points near the interface based on their original grayscale pixel intensity values. The distortions of FE elements, if this happened as a result of the initial nodal relocation, were corrected and refined (see section 6.3 for details). The resulting 3D FE mesh (e. g. , Figures 6.9 and 6.10) demonstrates reasonable acinar volume rendering.

Finally, for the type of image analysis discussed in this paper, the need for massive computation for 3D rendering as well as subsequent FE computational analyses is unavoidable. In this regard, improving our computational capability, particularly by using parallel and grid computing algorithms, would be required in the near future.

\subsection{ACKNOWLEDGMENTS}

We thank F. Marone and C. Hintermüller (Swiss Light Source, Paul Scherrer Institute) for expert help at the Beamline; B. de Breuyin, K. Sala-Szymanska, and B. Haenni for the embedding and preparation of the samples; C. Lehmann for the tricky shaping of the samples on the lathe; and D. Petrovic for preparing Figure 6.10. We also thank J. P. Butler and S. Tschanz for useful discussion on the subject of stereology.

This work was supported by National Heart, Lung, and Blood Institute Grants HL-054885, HL-070542, and HL-074022, Serbian Ministry of Science, OI144028, TR12007, and Swiss National Science Foundation Grant 3100Ao-109874/1. 
RADIATION DOSE OPTIMIZED LATERAL MICROSCOPY

David Haberthür ${ }^{1,2}$

Christoph Hintermüller ${ }^{3,4}$

Federica Marone ${ }^{3}$

Johannes C. Schittny ${ }^{1}$

Marco Stampanoni $3,4,5$

First published in: J. Synchrotron Rad. (2010). 17.

doi:10.1107/Sog09049510019618

\subsection{ABSTRACT}

Volumetric data at micrometer level resolution can be acquired within a few minutes using synchrotron radiation based tomographic microscopy. The field of view along the rotation axis of the sample can easily be increased by stacking several tomograms, allowing the investigation of long and thin objects at high resolution. On the contrary, an extension of the field of view in the perpendicular direction is non trivial. This paper presents an acquisition protocol which increases the field of view of the tomographic dataset perpendicular to its rotation axis. The acquisition protocol can be tuned as a function of the reconstruction quality and scanning time. Since the scanning time is proportional to the radiation dose imparted to the sample, this method can be used to increase the field of view of tomographic microscopy instruments while optimizing the radiation dose for radiation sensitive samples and keeping the quality of the tomographic dataset on the required level. This approach, dubbed wide field synchrotron radiation tomographic microscopy, can increase the lateral field of view up to five times. The method has been successfully applied for the three-dimensional imaging of entire rat lung acini with a diameter of $4.1 \mathrm{~mm}$ at a voxel size of $1.48 \mu \mathrm{m}$.

1 Institute of Anatomy, University of Bern, Bern, Switzerland

2 haberthuer@ana.unibe.ch

3 Swiss Light Source, Paul Scherrer Institut, Switzerland

4 Institute for Biomedical Engineering, University and ETH Zürich, Switzerland

5 marco.stampanoni@psi.ch 


\subsection{INTRODUCTION}

The functional respiratory lung unit-the so-called acinus-is defined as the complex of alveolated airways distal of a last purely conducting airway, the terminal bronchiole [28]. The total of all acini forms the lung parenchyma, the area where the pulmonary gas-exchange takes place. While the structural development of the gas-exchange region including the alveolar septa is quite well characterized [11, 17, 18], the development of the three-dimensional structure of its functional unit-of the acini-was not much studied due to the lack of suitable methods.

It is our goal to study the branching pattern of the acinar airways as well as the airflow within it. Tomographic methods, in particular synchrotron radiation based tomographic microscopy can access this kind of information nondestructively and noninvasively.

In order to visualize the thin sheets of tissue (alveolar septa) forming the gas-exchanging alveoli, a resolution in the order of one micron is required. An entire acinus is usually larger than the field of view of the tomographic microscope [10, 28], being the latest limited by the chosen optical configuration. Usually, a large field of view resulting in a large sample volume can only be acquired with low magnification and vice-versa. Lab-based micro-Computed Tomography $(\mu \mathrm{CT})$ stations could potentially be used to study acini, but the resolution of such systems is too low to resolve all alveolar septa. Even if $\mu \mathrm{CT}$ stations are catching up, synchrotron radiation based tomographic microscopy beamlines provide the necessary high resolution combined with unmatched image quality.

Up to now, the price to pay for this high resolution was a limited field of view. For instance at the beamline for TOmographic Microscopy and Coherent rAdiology experimenTs (TOMCAT) [61] at the Swiss Light Source, Paul Scherrer Institute, Villigen, Switzerland, the field of view at a $10 \times$ magnification ( $0.74 \mu \mathrm{m}$ voxel size) is limited to $1.52 \times 1.52 \mathrm{~mm}$, insufficient for the imaging of an entire acini at high resolution.

Increasing the field of view perpendicular to the rotation axis of the sample cannot easily be achieved by placing tomographic datasets next to each other. It is instead necessary to merge several projections overlapping the desired field of view prior to tomographic reconstruction. Obviously, to satisfy the sampling theorem, increasing the field of view also requires to acquire more projections, finally resulting in an increased acquisition time.

We developed such a method to merge several independently acquired sets of projections to increase the field of view of the resulting tomographic dataset. In addition, by optimization of the number of recorded projections, we established different scanning protocols with a user-defined balance between acquisition time and image quality.

Because the total acquisition time is directly linked to the radiation imparted to the sample, it is obvious that such protocols also affect radiation damage and constitute an important optimization tool for radiation sensitive experiments. 


\subsection{MATERIALS AND METHODS}

\subsubsection{Sample Preparation}

Rat lung samples, prepared according to Tschanz and Burri [123] and Luyet et al. [124] were used as test objects. Briefly, lungs of SpragueDawley rats were filled with $2.5 \%$ glutaraldehyde $\left(\mathrm{CH}_{2}\left(\mathrm{CH}_{2} \mathrm{CHO}\right)_{2}\right)$ in $0.03 \mathrm{M}$ potassium-phosphate buffer $(\mathrm{pH} 7.4)$ by instillation via tracheotomy at a constant pressure of $20 \mathrm{~cm}$ water column. In order to prevent recoiling of the lung, this pressure was maintained during glutaraldehyde-fixation for a minimum of two hours. Subsequently, the lungs were dissected free and immersed in toto in the same fixative at a temperature of $4{ }^{\circ} \mathrm{C}$ for at least $24 \mathrm{~h}$.

The samples were postfixed with $1 \%$ osmium tetroxide $\left(\mathrm{OsO}_{4}\right)$ and stained with $4 \%$ uranyl nitrate $\left(\mathrm{UO}_{2}\left(\mathrm{NO}_{3}\right)_{2}\right)$ to increase the x-ray absorption contrast, dehydrated in a graded series of ethanol and embedded in paraffin using Histoclear (Merck KGaA, Darmstadt, Germany) as an intermedium. The lung samples were mounted onto standard scanning electron microscopy sample holders (PLANO GmbH, Wetzlar, Germany) using paraffin [19].

The handling of animals before and during the experiments, as well as the experiments themselves, were approved and supervised by the Swiss Agency for the Environment, Forests and Landscape and the Veterinary Service of the Canton of Bern, Switzerland.

\subsubsection{Synchrotron radiation tomographic microscopy}

The experiments were performed at the TOMCAT beamline at the Swiss Light Source, Paul Scherrer Institut, Villigen, Switzerland. The samples were scanned at $12.6 \mathrm{keV}$. After penetration through the sample, the $\mathrm{x}$-rays were converted into visible light by a YAG:Ce scintillator (18 $\mu \mathrm{m}$ thickness, Crismatec Saint-Gobain, Nemours, France). Projections were magnified by diffraction limited microscope optics (10 $\times$ magnification) and digitized by a high-resolution $2048 \times 2048$ pixel CCD camera (pco.200o, PCO AG, Kelheim, Germany) with 14 bit dynamic range. The detector was operated in $2 \times 2$ binning mode. As a result, the pixel size was $1.48 \mu \mathrm{m}$ and the exposure time was $175 \mathrm{~ms}$.

Projections $I_{P r}$ were recorded at equiangular positions between $o^{\circ}$ and $180^{\circ}$. The exact number of angular projections depended on the selected scan protocol, as described in subsection 7.3.3. Additionally, for each protocol a set of dark $\left(I_{D}\right)$ and flat images $\left(I_{F}\right)$ were recorded for noise and baseline correction, respectively. Technical specifications of the beamline setup can be found in [38], the complete imaging and reconstruction workflow is described in [36].

\subsubsection{Increasing the field of view}

For parallel beam geometry, tomographic images are obtained at equidistant angles over a sample rotation of $180^{\circ}$ as shown in Figure 7.1. After reconstruction, the width of the image corresponds to the field of view of the camera.

Samples twice as large as the field of view can be imaged using scanning protocols based on a $360^{\circ}$ off center sample rotation as shown in Figure 7.1b. Images recorded between $180^{\circ}$ and $360^{\circ}$ have to be 
flipped after acquisition: the projections obtained at angular position $\theta$ and $\theta+180^{\circ}\left(\mathrm{I}_{\mathrm{Pr}_{\theta}}\right.$ and $\left.\mathrm{I}_{\operatorname{Pr}_{\theta+180^{\circ}}}\right)$ have to be stitched to one projection. The resulting images cover twice the field of view of the camera.

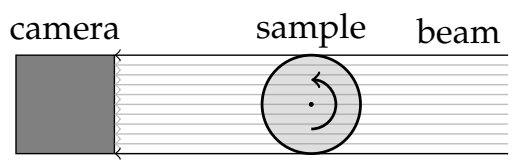

(a) One $180^{\circ}$ scan

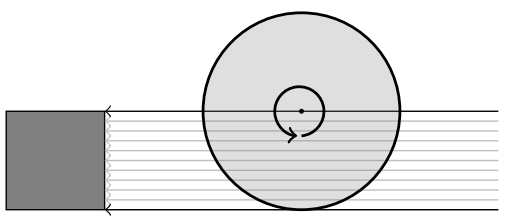

(b) One $360^{\circ}$ scan

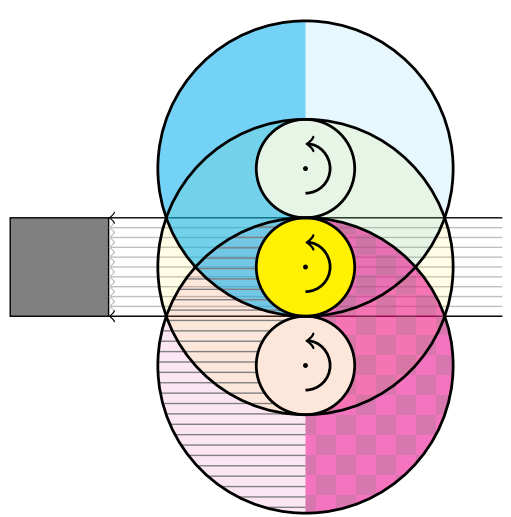

(c) Wide field scanning

Figure 7.1: Covering the field of view of differently sized samples with one $180^{\circ}$ scan (a), one $360^{\circ}$ scan (a) or-in the case of the so called wide field scanning-with multiple subscans (three subscans, (c)). The filled segments mark the region of the sample that is covered while scanning the respective positions (Position 1: magenta/checkerboard, Position 2: yellow, Position 3: cyan/striped).

For tomographic scans covering a size wider than two fields of view, three or more $180^{\circ}$-scans taken at slightly overlapping positions are combined, as shown in Figure 7.1c. The projections of each subscan overlap slightly to facilitate the stitching of multiple projections into a single one. The cutline, i. e. the position where the merging takes place, is automatically determined according to a mean squared difference method [36].

A straightforward acquisition scheme would record an equal amount of projections for each of the individual subscans. As a consequence, to fulfill the sampling theorem in the lateral parts of the sample, oversampling the central parts of the sample would be necessary.

Since the total acquisition time per sample linearly scales with the total amount of recorded projections such an acquisition scheme obviously increases the total amount of beamtime for one sample without relevantly increasing the quality of the reconstructed tomographic data. Hence, such an oversampling is generally avoided.

Our goal was to find a good compromise between scanning time and image quality. We therefore devised an acquisition scheme for covering a wide field of view based on the assumption that a sufficient 
resolution and contrast can be achieved in the tomographic dataset, if the sampling theorem is individually fulfilled for each of the subscans. This results in a set of $i$ subscans with $P_{i}$ projections each. A simple example with $P_{2}=4$ and $P_{1}=P_{3}=8$ is shown in Figure 7.2a. Since each subscan $i$ has a different number of projections $P_{i}$, the stitching algorithm has to interpolate missing projections from adjacent projections (represented by the dotted lines in Figure 7.2b) to generate a complete set of merged projections for reconstruction.

As a by-product, such an optimization of the individual number of projections $P_{i}$ for each subscan $i$ decreases the total acquisition time for one sample and thus the imparted radiation dose.

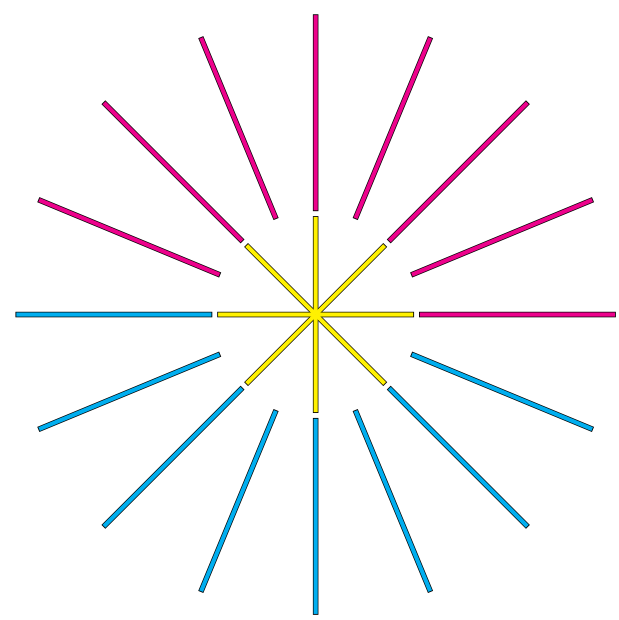

(a) scanned projections

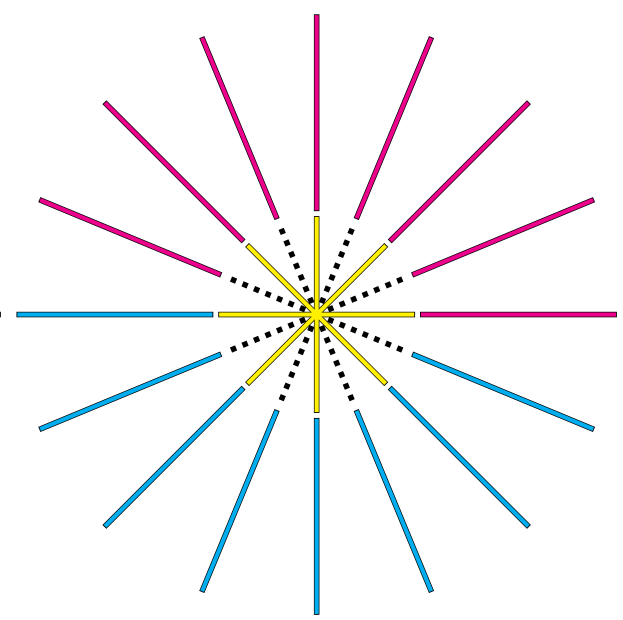

(b) scanned and interpolated projections

Figure 7.2: Wide field scan setup with three $180^{\circ}$ scans; one central (yellow) and two lateral scans (magenta and cyan or top and bottom, respectively). In this drawing, four projections for the central and eight projections for each of the lateral scans have been recorded. The colors of the three positions correspond to the colors shown in Figure 7.1c. 7.2a: scanned projections 7.2b: scanned projections and additional interpolated projections (dotted) required to merge all projections.

We defined a gold standard protocol and several additional scanning protocols in order to compare different acquisition schemes. The gold standard protocol covers the desired field of view while fulfilling the sampling theorem-which states that for a detector width of D pixels, we need to acquire a number of projections $P=D \pi / 2[51]-i n$ all its regions, as shown in Figure 7.3a. In this case we need to achieve a field of view of 3072 pixels. The dark gray circle is the field of view that could be covered using a large detector with a size of 3072 pixels and recording $\mathrm{P}=3072 \pi / 2=4825$ projections.

Using a detector with a size of 1024 pixels, this desired field of view could be covered with nine independent local tomography scans. Such an approach would require nine independent reconstructions and stitching of those nine reconstructed tomographic datasets into one dataset covering the full field of view. This method would also introduce artifacts at the edges of each of the nine sub-datasets which would lie inside the sample to be imaged.

While the chosen field of view of $3072 \times 3072$ pixels can be covered using a detector of the size of 3072 pixels in one scan, we can cover the 
desired field of view with a much smaller detector, using a scanning protocol with three subscans from which we obtain merged projections. Figure $7.3 \mathrm{~b}$ shows how the desired field of view of 3072 pixels can be covered with a wide field scan, composed of one central and two half ring-scans, recorded with a small detector with a size of 1024 pixels and 4825 projections per subscan (a total of 14475 projections) which are then subsequently merged to 4825 large projections spanning the whole field of view. A further increase in the field of view can be obtained by simple iteration. Figures $7.3 \mathrm{c}-7.3 \mathrm{f}$ show such a setup for a five- or seven-fold increase.

\subsubsection{Quality guided protocols}

Taking the experimental constraints like desired field of view, available detector size, magnification and binning into account, a MATLABscript calculates a set of acquisition protocols. Each such protocol contains the number of projections for each subscan linearly scaled in total amount of projections from a gold standard scan down to a protocol where the sampling theorem is far from being satisfied (Table 7.1). Through optimization of the number of recorded projections, a reduction of the total acquisition time by $84 \%$ (compared to the gold standard) was achieved.

Using a Shepp-Logan phantom [125] with added Gaussian noise as a reference image, a simulated tomographic scan and subsequent reconstruction was calculated for each of these acquisition protocols. For each protocol we calculated the expected reconstruction quality using the difference image between the reconstruction of this protocol and the initial reference image. This simulated reconstruction quality was plotted against the total acquisition time (red dots in Figure 7.6).

The end-user-balancing between acquisition time and desired image quality-chooses one protocol from the presented set for scanning his sample. A file containing all the details of the chosen scan is written to disk, and parsed by a custom Python-script. This script interacts with the hardware control system at the TOMCAT beamline enabling an automated, unattended batch acquisition of all necessary subscans.

To assess the simulations in a real world example, we selected nineteen different acquisition protocols with varying number of projections to scan one single sample (details are specified in Table 7.1, including the calculated Quality for each protocol).

A scan covering the chosen field of view with nine independent local tomography scans, each with a field of view of $1024 \times 1024$ pixels, would need a total of $\mathrm{P}=9(1024 \pi / 2)=14476$ projections. This protocol was not considered for this study, since the sampling theorem can be equally satisfied by acquiring the required amount of projections with one central and two ring scans, as defined in subsection 7.3.3. Including an overlap of 100 pixels between the central and the ring scan, an equivalent wide field scanning protocol (Protocol A in Table 7.1) requires the acquisition of 13534 projections $\left(P_{A}=3(3072-200) \pi / 2\right)$.

Protocols B-T have been linearly scaled down with a decreasing number of acquired projections of the ring scans. To simplify interpolation and merging of the projections from each subscan, we only selected acquisition schemes where the number of projections of the inner and the outer subscans is the same or a multiple of two (see Figure 7.2). This constraint also led to a slight oversampling for pro- 


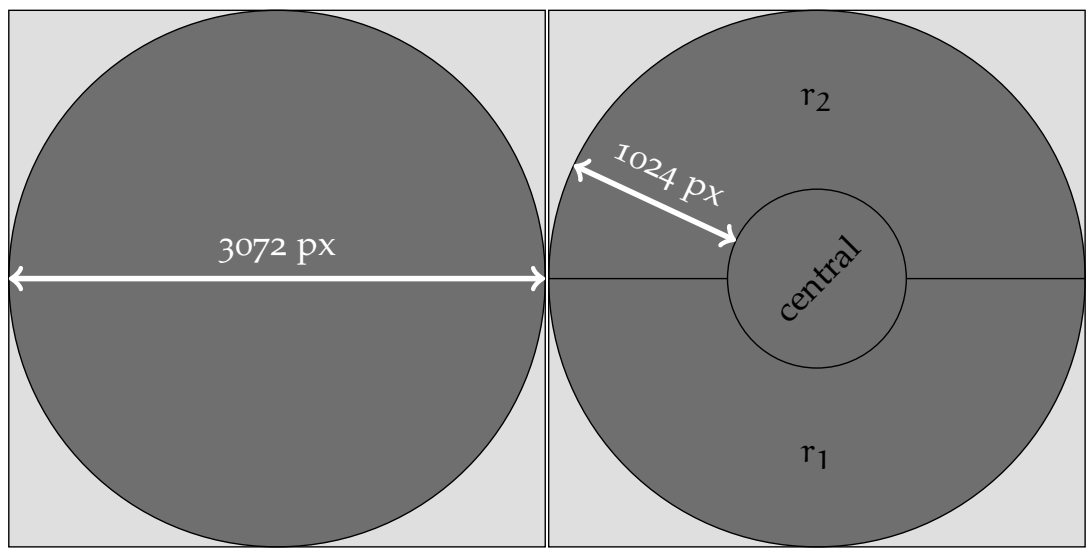

(a)

(b)

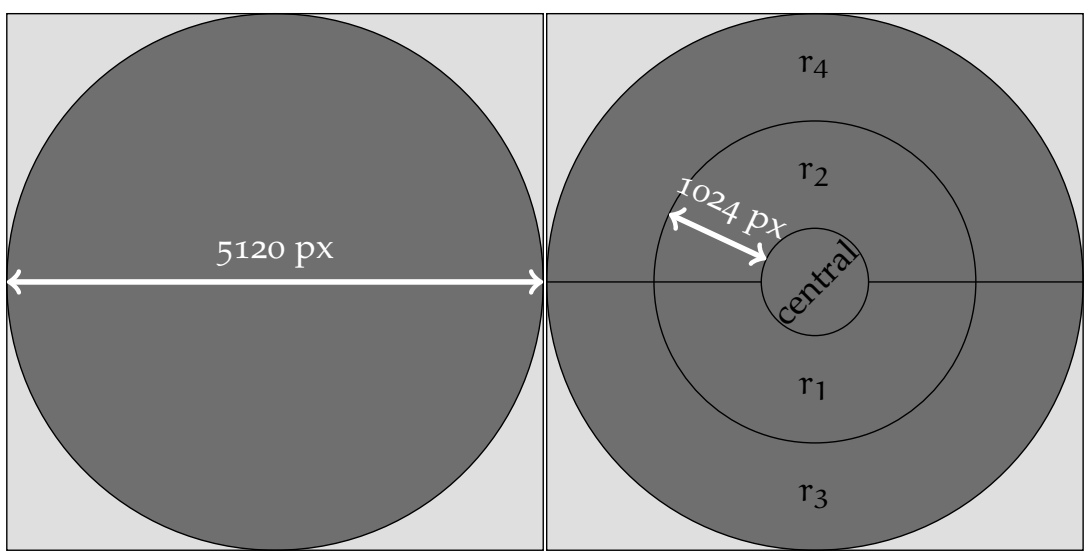

(c)

(d)

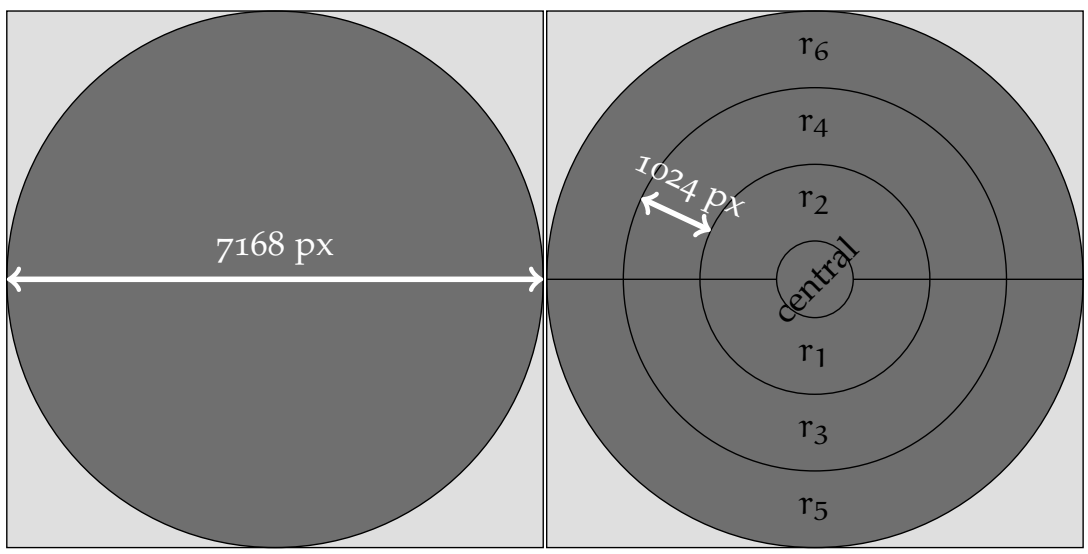

(e)

(f)

Figure 7.3: Setup for different field of views. (a): Desired field of view of 3072 pixel diameter. (b): Wide field scanning protocol for covering the desired field of view of panel (a) with merged projections from one central and two half ring scans $\left(r_{1}\right.$ and $\left.r_{2}\right)$. (c): Desired field of view of 5120 pixel diameter. (d): Wide field scanning protocol for covering the desired field of view of panel (c) with merged projections from one central and four half ring scans $\left(r_{1}-r_{4}\right)$. (e): Desired field of view of 7168 pixel diameter. $(\mathrm{f})$ : Wide field scanning protocol for covering the desired field of view of panel (e) with merged projections from one central and six half ring scans $\left(r_{1}-r_{6}\right)$. 
tocol B, otherwise the number of projections for each subscan of this protocol $(5244=3 \cdot 874)$ would not have scaled down nicely to the 874 projections used for protocol T.

All parameters of each protocol and each subscan (sample-position in relation to the beam, rotation angles and number of projections) were set in a preference-file, generated using aforementioned MATLABscript. One rat lung sample was scanned using each of the 19 different protocols (B-T), without manual intervention, permitting a direct comparison of the reconstructed datasets.

Table 7.1: Details of the 19 scanned protocols for this study (B-T): An unoptimized scan to cover the desired field of view of 3072 pixels with nine independent scans (with a detector width of 1024 pixels) would require to record a total of $P_{\text {Gold standard }}=9(1024) \pi / 2=$ 14476 projections. The wide field scanning protocol (A) equivalent to this field of view only uses three subscans, resulting in a total number of projections of $P_{A}=$ $3(3072-200) \pi / 2=13534$. Three-dimensional reconstructions of the datasets marked with a light gray background are shown in Figure 7.7.

\begin{tabular}{|c|c|c|c|c|c|c|}
\hline \multirow{2}{*}{ Protocol } & \multicolumn{3}{|c|}{ Projections for Subscan } & \multirow{2}{*}{$\begin{array}{l}\text { Total Number } \\
\text { of Projections }\end{array}$} & \multirow{2}{*}{$\begin{array}{c}\text { Time/Radiation } \\
\text { Dose [\%] }\end{array}$} & \multirow{2}{*}{$\begin{array}{l}\text { Simulated } \\
\text { Quality [\%] }\end{array}$} \\
\hline & $\mathrm{s}_{1}$ & $\mathrm{~s}_{2}$ & $\mathrm{~s}_{3}$ & & & \\
\hline $\mathrm{A}^{1}$ & & & & 13534 & 100 & \\
\hline $\mathrm{B}^{2}$ & 5244 & 5244 & 5244 & 15732 & 116 & 100 \\
\hline $\mathrm{C}$ & 5244 & 2622 & 5244 & 13110 & 97 & 89 \\
\hline $\mathrm{D}$ & 4370 & 4370 & 4370 & 13110 & 97 & 85 \\
\hline $\mathrm{E}$ & 4370 & 2185 & 4370 & 10925 & 81 & 87 \\
\hline $\mathrm{F}$ & 3934 & 3934 & 3934 & 11802 & 87 & 80 \\
\hline G & 3934 & 1967 & 3934 & 9835 & 73 & 84 \\
\hline $\mathrm{H}$ & 3496 & 3496 & 3496 & 10488 & 77 & 78 \\
\hline $\mathrm{I}$ & 3496 & 1748 & 3496 & 8740 & 65 & 80 \\
\hline $\mathrm{J}$ & 3060 & 3060 & 3060 & 9180 & 68 & 76 \\
\hline K & 3060 & 1530 & 3060 & 7650 & 57 & 75 \\
\hline $\mathrm{L}$ & 2622 & 2622 & 2622 & 7866 & 58 & 72 \\
\hline $\mathrm{M}$ & 2622 & 1311 & 2622 & 6555 & 48 & 69 \\
\hline $\mathrm{N}$ & 2186 & 2186 & 2186 & 6558 & 48 & 67 \\
\hline $\mathrm{O}$ & 2185 & 1093 & 2185 & 5463 & 40 & 62 \\
\hline $\mathrm{P}$ & 1748 & 1748 & 1748 & 5244 & 39 & 61 \\
\hline $\mathrm{Q}$ & 1748 & 874 & 1748 & 4370 & 32 & 55 \\
\hline $\mathrm{R}$ & 1312 & 1312 & 1312 & 3936 & 29 & 46 \\
\hline$S$ & 874 & 874 & 874 & 2622 & 19 & 21 \\
\hline $\mathrm{T}$ & 874 & 437 & 874 & 2185 & 16 & 20 \\
\hline
\end{tabular}

\subsubsection{Projection merging and tomographic reconstruction}

After acquisition of the three subscans per protocol, custom MATLAB functions read the parameters of the single subscans (e.g. sample name, amount of subscans, amount of dark and flat images) as well as the desired output-name and -suffix, and performed all necessary calculations, including: loading of the correct projections from each subscan; normalizing; interpolation; cutline detection; correct stitching of the images into wide field projections, and writing these merged projections as well as $\log$ files needed for the reconstruction to disk. 
The merged projections were subsequently rearranged into sinograms, where the $n^{\text {th }}$ sinogram is composed of the $n^{\text {th }}$ line of every corrected projection. The $\mathrm{n}^{\text {th }}$ slice of the tomographic scan was reconstructed from the $n^{\text {th }}$ sinogram using an FFT-based regridding algorithm [126, 127]. The 19 tomographic datasets were reconstructed on a computing cluster composed of five 64 bit Opteron machines with four cores and 8 GB RAM each. The reconstructions resulted in an image stack covering a large sample volume of $2792 \times 2792 \times 1024$ pixels, a nine-fold increase from the standard volume of $1024 \times 1024 \times 1024$ pixels for one conventional scan.

\section{$7 \cdot 4$ RESULTS}

\subsubsection{Image Merging and Reconstruction}

Figure 7.4a shows corrected projections from three overlapping subscans prior to merging, including regions where the subscans are overlapping. Figure $7.4 \mathrm{~b}$ shows one merged projection prior to reconstruction and Figure 7.4c shows one slice of the reconstructed dataset. The example shown in Figure 7.4 was obtained using the highest number of projections and is therefore protocol $\mathrm{B}$. One reconstructed slice covers a field of view of $2792 \times 2792$ pixels $(4.13 \times 4.13 \mathrm{~mm})$, which is almost three times the size of what can be achieved with one single binned scan (1024 pixels or $1.52 \mathrm{~mm}$ ). The dashed circles on the reconstructed slice mark the start and the end of the overlap region.

Figure 7.5 shows the advantages of the wide field acquisition scheme. With - in this particular case-an enlargement of the field of view by almost a factor of three, it is possible to visualize entire acini at high resolution. For a conventional scan (Figure 7.5a), the airway segments in the sample are only partially contained inside the dataset (magenta and yellow). The semitransparent airway segments are contained in the sample, but are not visible in the field of view of a dataset obtained with a conventional scan. Increasing the field of view (Figure 7.5b) allows the visualization of those segments to their full extent. A third acinus (cyan) which was not visible in Figure 7.5a can now easily be visualized.

\subsubsection{Performance of the scanned protocols}

The performance of the 19 protocols has been quantified using the difference image between binarized slices of the gold standard protocol and each protocol to be assessed. The slices have been thresholded according to Otsu [128]. The difference value $\left(E_{\text {norm }}\right)$ plotted in Figure 7.6 was calculated for each protocol $i=1-19(B-T)$ according to equations 7.1-7.3. Using a thresholded slice $k$ of each protocol $i$ $\left(\right.$ Slice $\left._{i_{k}}\right)$ and the corresponding slice $k$ of the gold standard protocol $B$ (Slice $\left.B_{k}\right)$ the absolute difference image $\left(D_{i_{k}}\right)$ of these two slices $k$ was calculated. The sum of all pixels of this difference image yields a 


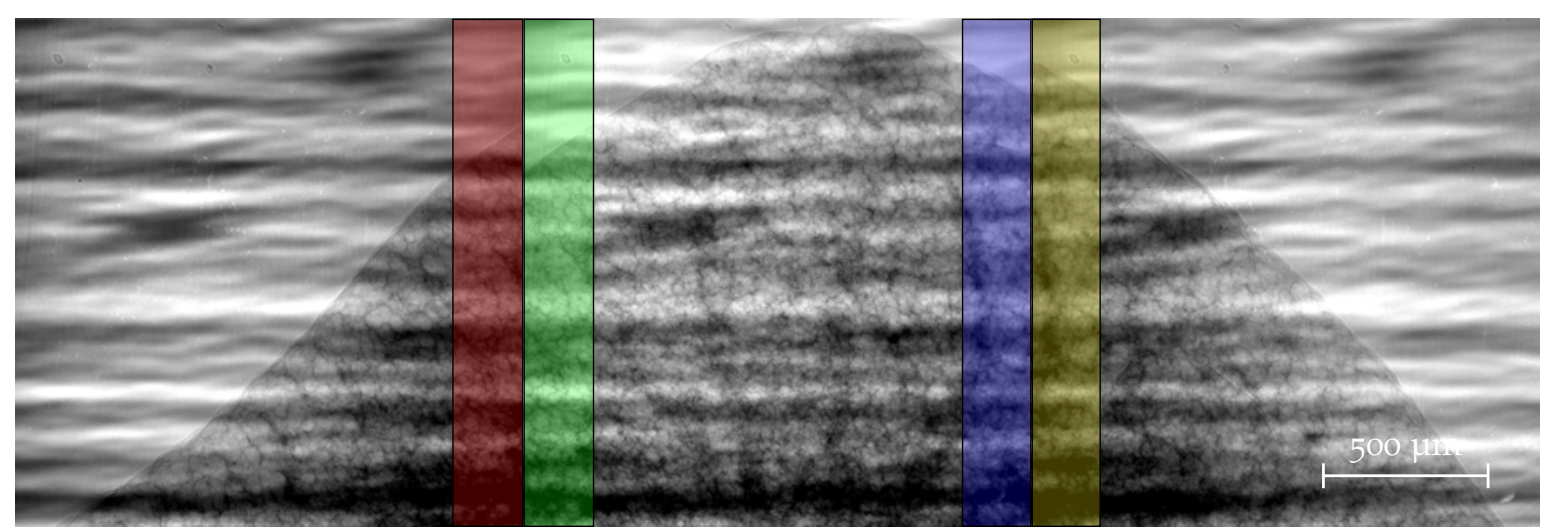

(a) Projections from subscans

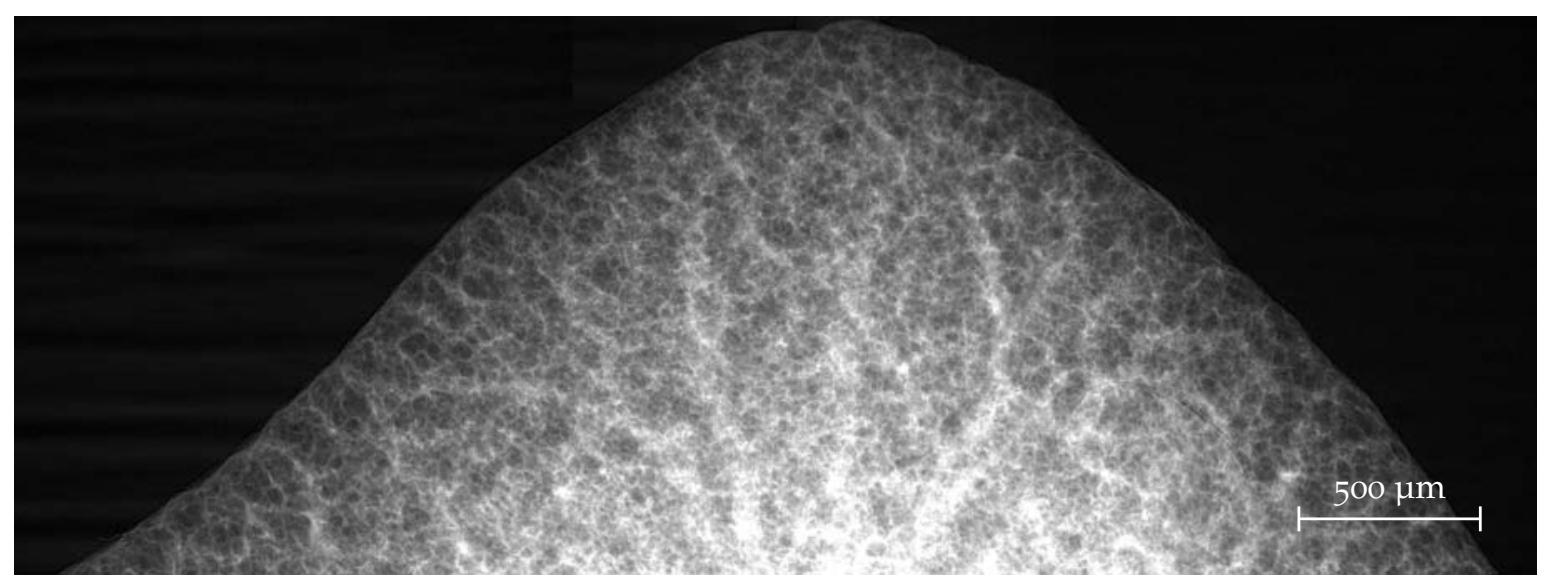

(b) Merged and corrected projection

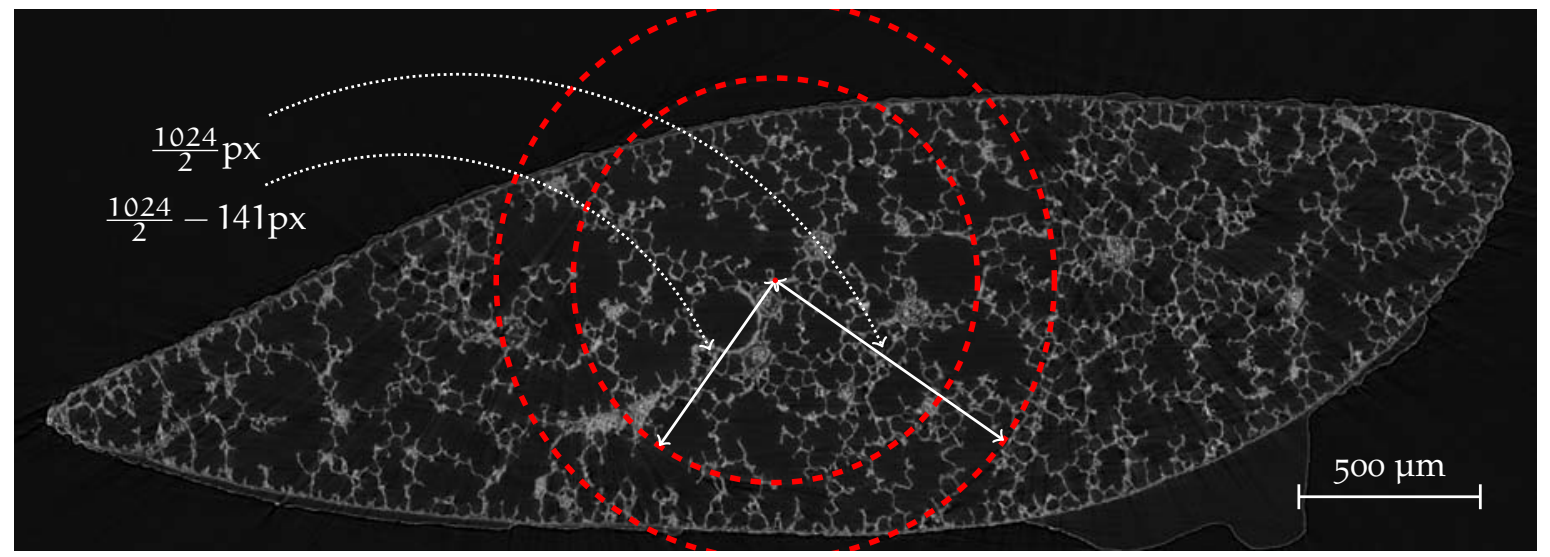

(c) Reconstruction

Figure 7.4: Workflow of a wide field scan. The images show a rat lung sample from a Sprague-Dawley rat, obtained 21 days after birth, scanned with the acquisition protocol B (see Table 7.1). (a): Three corrected and independently acquired projections from subscans $s_{1}-s_{3}$ are shown. Each one is $1024 \times 1024$ pixels large and covers a field of view of $1.52 \mathrm{~mm}$. Subscans $s_{1}$ and $s_{2}$ overlap by 141 pixels (red and green overlay), subscans $s_{2}$ and $s_{3}$ overlap by 138 pixels (blue and yellow overlay). (b): Merged projection obtained from the three subscans shown in subfigure (a). Each merged projection has a size of $2792 \times 1024$ pixels. Due to the overlap required to merge the projections, the width of the merged projections is slightly smaller than three times the width of the subscans. (c): Cropped slice of the reconstructed tomographic dataset. The dashed red circles mark the start and end of the overlap region. 


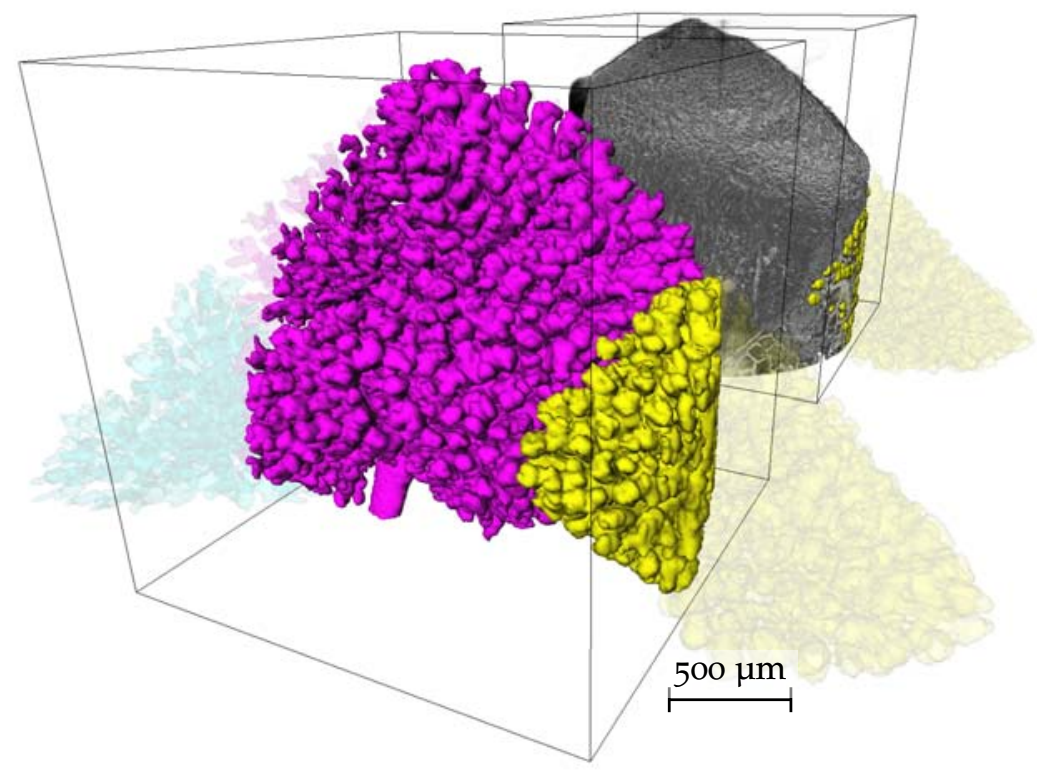

(a) Conventional scan

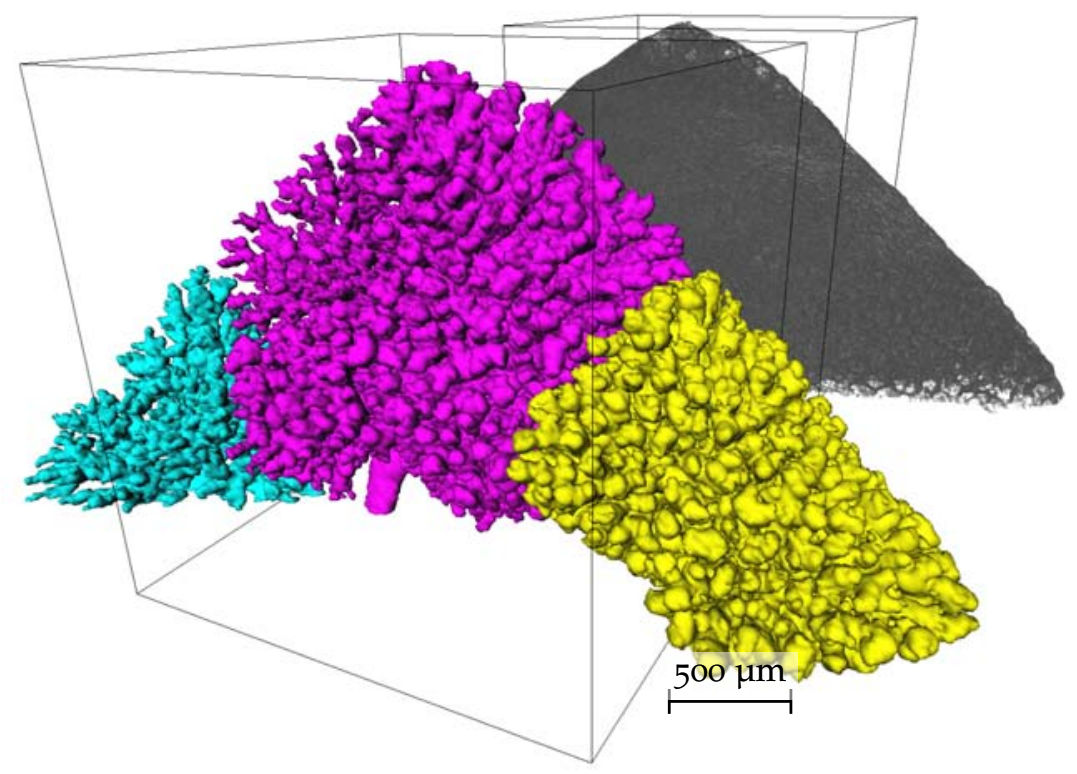

(b) Wide field scan

Figure 7.5: Three-dimensional visualization of the distal-medial tip of the right lower rat lung lobe. The gray structure in the background shows a semitransparent view of the tomographic dataset with segmented airways. The foreground shows isosurfaces of terminal airways. The wireframe cube has a side length of 1024 pixels and encloses the field of view of one conventional scan. (a): Conventional scan; the extracted airway segments (magenta and yellow or left and right, respectively) are only partially contained inside the total sample volume. Airway segments not contained in the dataset, but present in the sample are shown semitransparent. This conventional scan corresponds to a reconstruction of the central of the three wide field scan subscans. (b): Wide field scan with increased field of view; the magenta (center) and yellow segment (right) show entire acini inside the dataset, the cyan segment (left) contains a partially cut acinus. All airway segments inside the sample are contained in the tomographic dataset. 
value $\left(E_{i_{\text {norm }}}\right)$ for the difference of the examined slice $k$ of protocol $i$ with the corresponding slice of the gold standard protocol B.

$$
\begin{aligned}
D_{i_{k}} & =\mid \text { Slice }_{\mathrm{B}_{k}}-\text { Slice }_{i_{k}} \mid \\
E_{i_{\text {norm }_{k}}} & =\sum_{x} \sum_{y} D_{i_{k}} \\
E_{i_{\text {norm }}} & =\overline{E_{i_{\text {norm }_{k}}}}
\end{aligned}
$$

This combined difference value $\left(\mathrm{E}_{\mathrm{i}_{\text {norm }_{\mathrm{k}}}}\right)$ was calculated for 205 regularly spaced slices (every fifth slice) of the full dataset. The mean $\left(\overline{E_{i_{\text {norm }_{k}}}}\right)$ difference value for all slices was normalized to the scanned quality-steps from $16-116 \%$ (as stated in Table 7.1) and plotted with its standard deviation $\left(\sigma\left(E_{i_{\text {norm }}}\right)\right)$. For the purpose of comparison, data has been normalized.

As expected, the calculated quality of the reconstructions representing the different protocols decreases as a function of total number of obtained projections (Figure 7.6). The calculated error of the different protocols (normalized difference value, blue diamonds) shows the experimental results obtained from actual scans of lung tissue. The plots for the simulation as defined in subsection 7.3.4 (red dots) and the normalized difference value are not perfectly in agreement, but show the same trend. The linear regression for the simulation shows a steeper decrease for the quality $\left(y_{\text {Sim }}=0.6936 x+26.891\right)$ than the linear interpolation for the experimental data $\left(y_{\operatorname{Exp}}=0.5833 x+20.226\right)$. The linear regression coefficient for both the linear interpolations are comparable $\left(R_{\text {Sim }}^{2}=0.8287, R_{\text {Exp }}^{2}=0.7868\right)$.

\subsubsection{Three-dimensional visualization of different protocols}

The tomograms of the different protocols were three-dimensionally analyzed and visualized using MeVisLab (Version 2.0 (2009-06-09 Release), MeVis Medical Solutions AG and Fraunhofer MEVIS - Institute for Medical Image Computing, Bremen, Germany). Airway segments were extracted using a threshold interval based region growing algorithm [129]. A seed point for the region growing algorithm was manually defined in the most proximal slice for each independent airway segment. The coordinates of the seed points were kept constant for protocol B-T, allowing direct comparison between the airway segment reconstructions of the different protocols. Airway segments extracted for protocol B, L and T are shown in Figure 7.7.

Protocol B corresponds to a slightly oversampled gold standard scan, obtained with total 15732 projections, recorded in $66 \mathrm{~min}$. Protocol L was obtained in $35 \mathrm{~min}$ with total 7866 projections. Protocol T was obtained in 12 min with 2185 projections for all three subscans. The tomographic dataset from protocol B was reconstructed from 5244 merged projections, the dataset from protocol L was reconstructed from 2622 merged projections and the dataset from protocol $\mathrm{T}$ was reconstructed using only 874 merged projections. Even though protocols L and T were scanned while violating the sampling theorem and with a total scanning time reduction of $40 \%(\mathrm{~L})$ or more than $86 \%(\mathrm{~T})$, the samples still appear to be identical to the gold standard protocol in the low-resolution three-dimensional visualizations shown in Figure $7 \cdot 7 \mathrm{a}-$ 7.7c.

Figure $7 \cdot 7 \mathrm{~d}-7 \cdot 7 \mathrm{f}$ show isosurface visualizations of the border between airspace and lung tissue as cubic regions of interest (256 pixels 


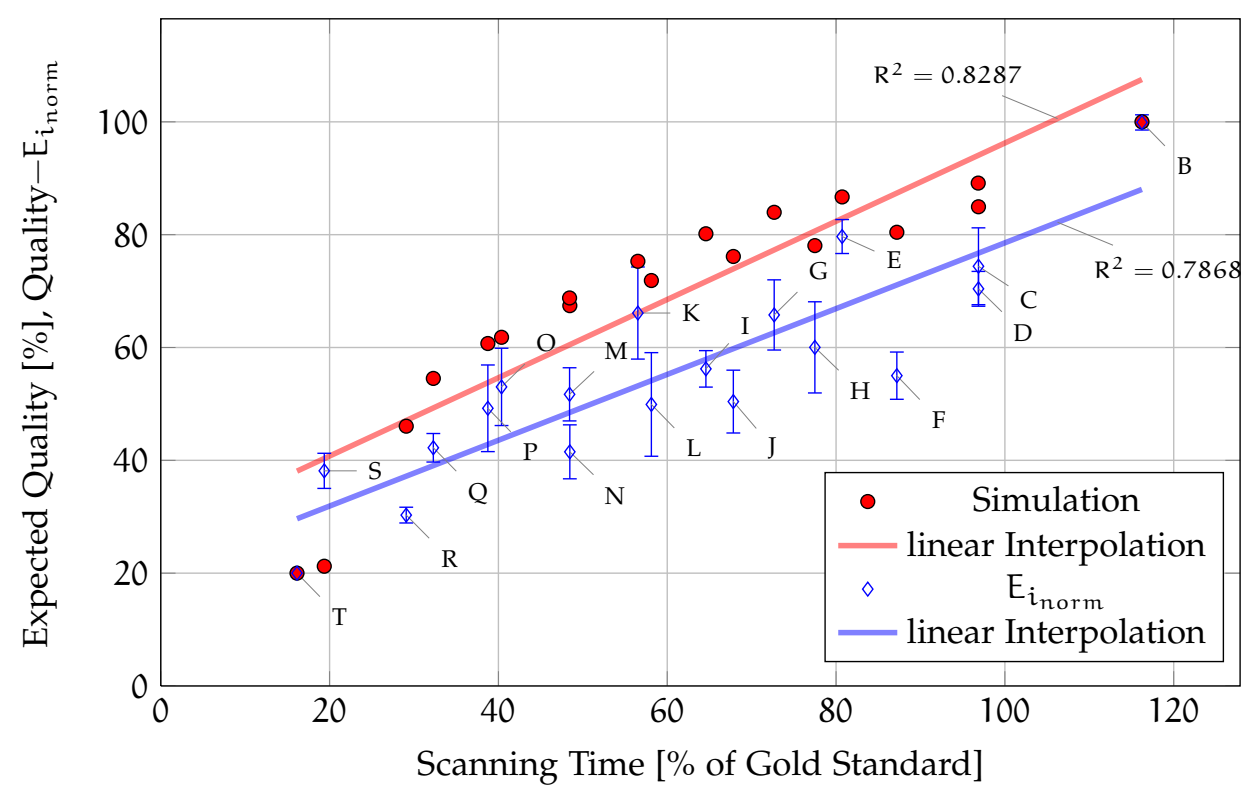

Figure 7.6: Plot of normalized difference Value ( $E_{i_{\text {norm }}}$, blue diamonds) for the 19 scanned protocols overlaid over Quality-plot (red dots) obtained from the simulation (described in subsection 7.3.4). The normalized Error has been calculated using the difference image of each protocol $i$ with protocol B. The error bars for each protocol show the standard deviation of the error calculated for 205 of the 1024 slices. Note that the scale of the error was normalized to 20$100 \%$, so that both the quality from the simulation and the error are directly comparable. The abscissa shows the scanning time in percentage of time used for the gold standard scan. Protocol T on the far left corresponds to the fastest scanning time, protocol B on the far right to the slowest. The protocols in between are shown from $\mathrm{T}-\mathrm{B}$ for increasing percentage of the scanning time.

wide, location inside the sample is marked as blue cube in Figure 7.7a7.7c. Because of experimental constraints, the cutline between the individual subscans could not be defined with a precision of one single pixel. As a consequence, the clipping plane does not lie in exactly the same position. This explains the appearing and disappearing holes in Figure $7 \cdot 7 \mathrm{~d}-7 \cdot 7 \mathrm{f}$.

Even with the higher magnification, the reconstruction of protocol L in Figure 7.7e appears nearly identical to the reconstruction of the region of interest of protocol B (Figure 7.7d). The isosurface of the region of interest of protocol T shown in Figure 7.7f appears rougher than the isosurface of protocol B. This roughness is introduced through ray-like artifacts visible in the original slice of the dataset of protocol $\mathrm{T}$ (not shown). These artifacts are the consequence of a strong subsampling. With the acquisition of only 874 projections instead of the required 5139, the sampling theorem is far from being satisfied. However, even with this strong undersampling, segmentation, three-dimensional reconstruction and visualization of the sample is still possible.

For further analysis four regions of interest with a side length of 256 pixels have been extracted for each of the protocols $\mathrm{B}, \mathrm{L}$ and $\mathrm{T}$. The three-dimensional location of these ROIs inside the sample is shown in Figure 7.8 . 


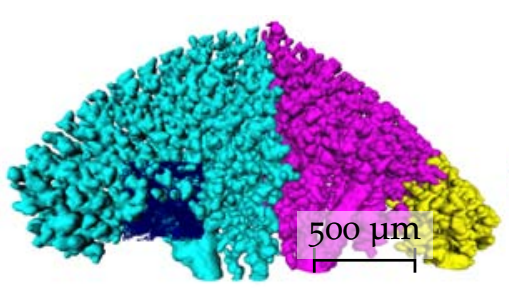

(a) Overview protocol B

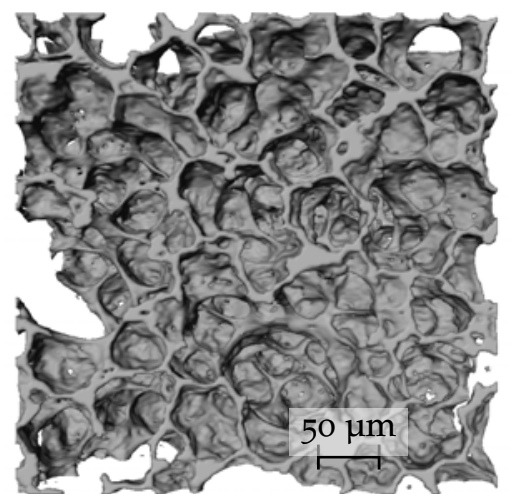

(d) ROI protocol B

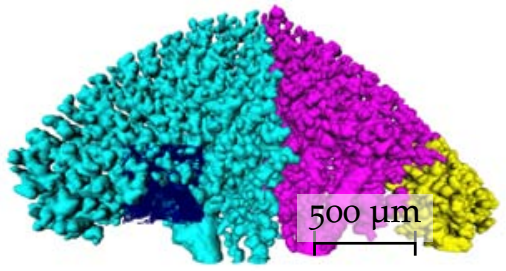

(b) Overview protocol L

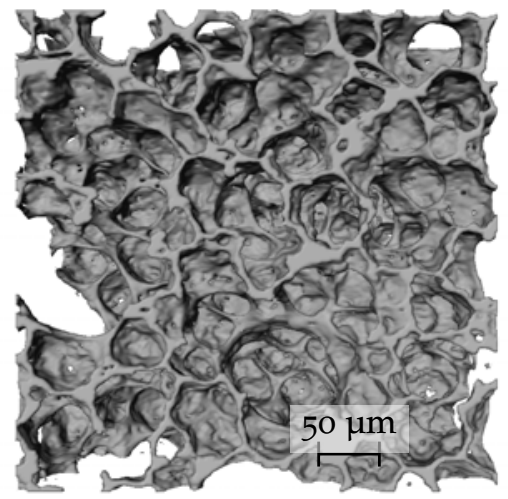

(e) ROI protocol L

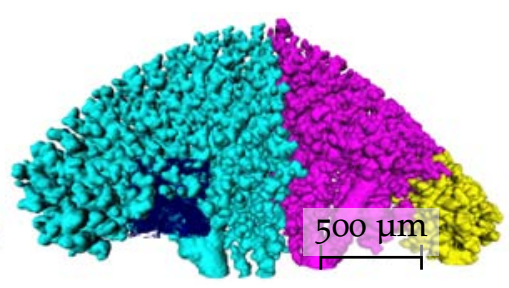

(c) Overview protocol T

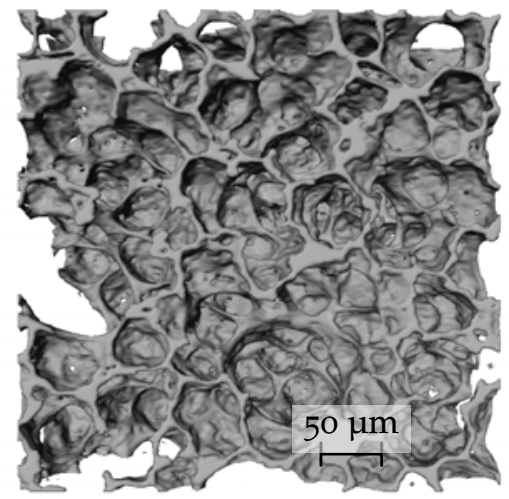

(f) ROI protocol T

Figure 7.7: Comparison of three-dimensional visualizations. (a)-(c): Three independent airway segments (cyan, magenta, yellow) of tomographic datasets obtained with protocol B, L and T, extracted using a region growing algorithm. A cubic region of interest (blue) with a side length of 256 pixels (corresponding to $379 \mu \mathrm{m}$ ) is marked inside the leftmost segment for all protocols. (d)-(f): Detailed view of isosurfaces of the lung tissue inside the blue ROIs for protocol B, $\mathrm{L}$ and $\mathrm{T}$, respectively. Note the increasing surface roughness in the alveolar surfaces for subfigures (e) and (f).

Each of the ROIs has been binarized using an algorithmically determined threshold [128] and small particles inside the segmented airspace lumen have been removed using a connected component analysis. Subsequently, the euclidean distance transformation [130] has been calculated for each thresholded ROI.

For comparison, the histogram of the euclidean distance transformation has been plotted for all four regions of interest in each protocol (B, L and T).

Figure 7.9 shows logarithmic plots of the histogram distributions for the four selected ROIs; the blue, green and red plot show the histograms of the distance transformation of Protocol B, L and T, respectively. For all four regions of interest, the distribution of the euclidean distance transformation is very similar, only for larger airway diameters (between 50-60 $\mu \mathrm{m}$ ) we see a detectable difference in the regions of interest one and four, located in the lateral parts of the sample. If we remember that the histogram is plotted with a logarithmic y-axis, we see that the difference of the histograms is only visible for several hundred voxels.

Even when reducing the sample acquisition time by $84 \%$ of the gold standard scan ( $\mathrm{T}$ vs. B), the distance transformation histograms of the shown regions of interest are very similar and therefore no relevant structural differences are introduced. 


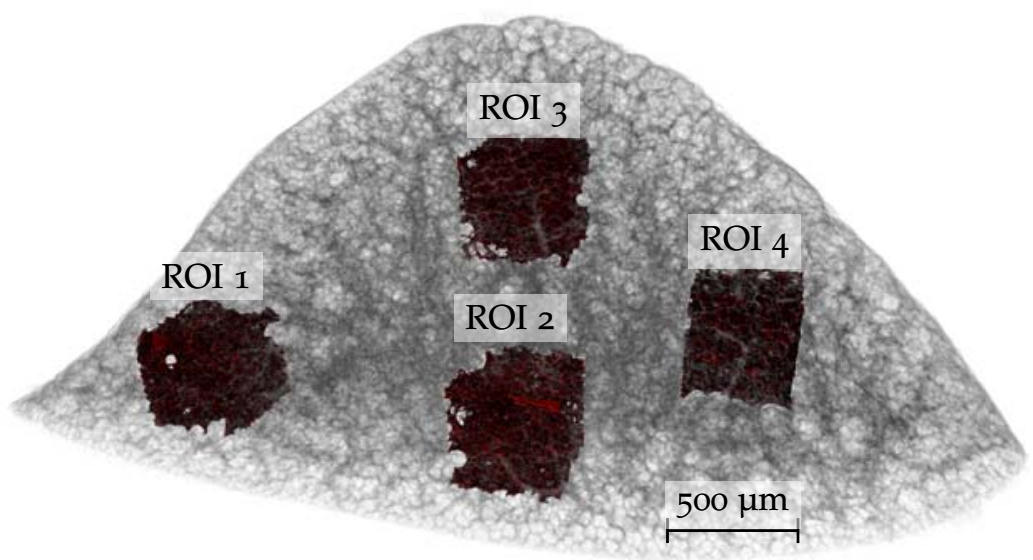

Figure 7.8: Overview of the location of the four regions of interest where the histogram of the euclidean distance transformation distribution has been calculated. Grey: Semitransparent volume rendering of the lung tissue sample. Red: Four regions of interest, extracted to calculate the distance transformation. The labels of the ROIs conform to the legends in Figure 7.9.

As a further proof of concept we scanned and reconstructed a rat lung sample with five scanning positions, resulting in a nearly five-fold $(4.74 \times)$ increase in field of view from slices with a size of $1024 \times 1024$ pixels to a size of $4852 \times 4852$ pixels $(1.52 \times 1.52 \mathrm{~mm}$ to $7.18 \times 7.18 \mathrm{~mm})$ at a voxel side length of $1.48 \mu \mathrm{m}$. A three-dimensional visualization of the boundary between airspace and tissue in this reconstructed dataset validated the wide field scanning method for further increases in the available field of view (data not shown).

\section{$7 \cdot 5$ DISCUSSION}

We present a method to laterally increase the field of view of tomographic imaging systems operated in parallel beam geometry and would like to call this method Wide Field Synchrotron Radiation Based X-ray Tomographic Microscopy (WF-SRXTM). We defined scanning protocols for the optimization of the total imaging time versus the expected imaging quality, enabling a very fast acquisition of lower quality tomographic datasets, or acquisition of very high quality datasets in a longer time.

Even if the reduction in scanning time does introduce minor artifacts in the three-dimensional reconstruction, as shown in Figure 7.7, an automated segmentation of the relevant features in the sample is still possible, even for protocols with greatly reduced scanning time.

The introduced artifacts in the three-dimensional reconstruction of the lung tissue are of small scale compared to the alveoli, the smallest structures we would like to visualize. At our scale, the structures which are in the range of our resolution are holes visible in the alveolar septa. Those holes may appear through the three-dimensional reconstruction at locations where the alveolar septa are too thin and/or the globally chosen threshold is too high. However, the observed holes are not exclusively artificial: Kohn [131] described micrometer sized pores-so-called pores of Kohn-located between adjacent alveoli, which can also be seen in rat lungs [132]. 

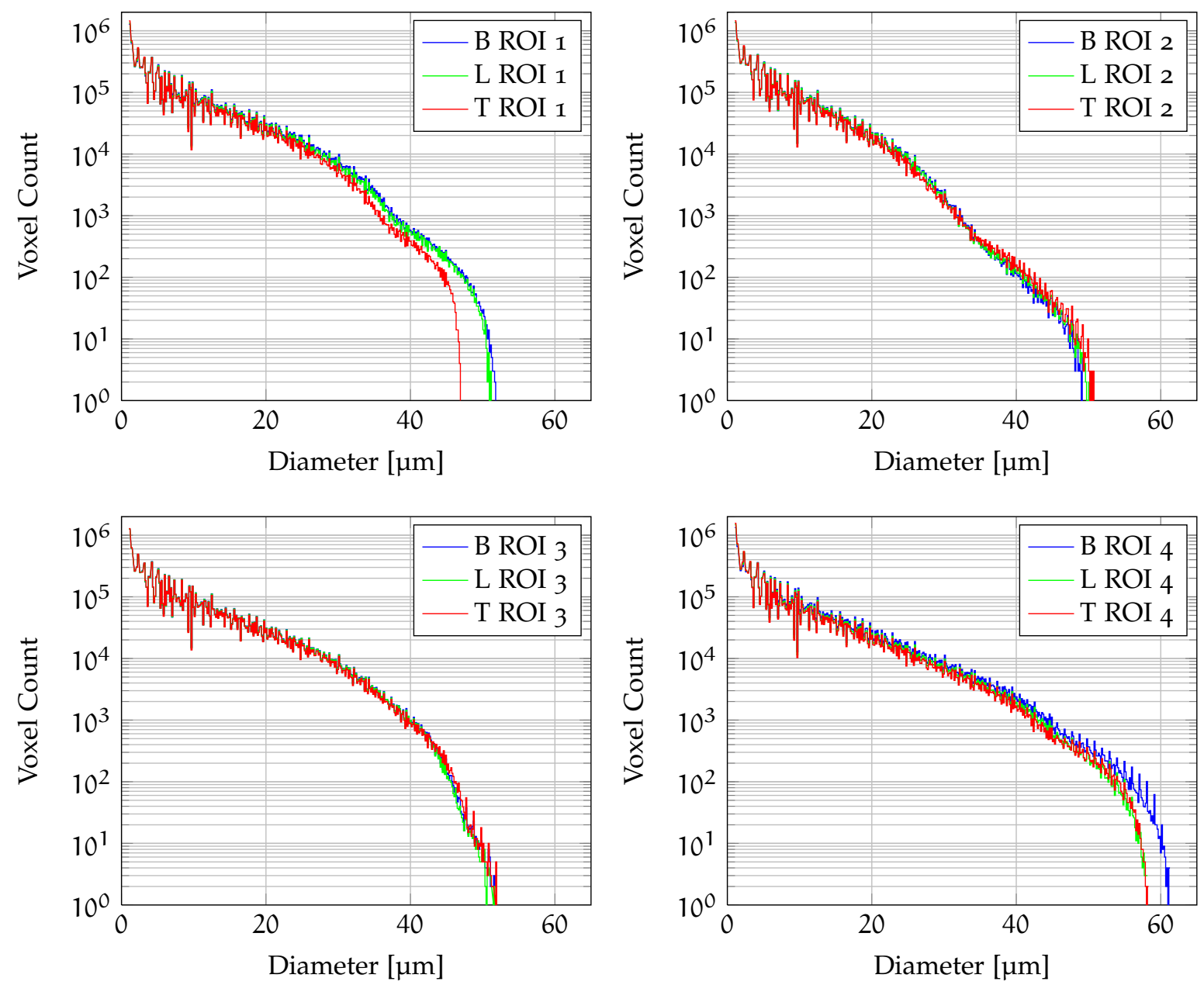

Figure 7.9: Histogram-Plots for each of the of four ROIs, each showing the histogram of the distance transformation for the protocols $\mathrm{B}, \mathrm{L}$ and $\mathrm{T}$.

Comparing the reconstructions shown in Figure $7.7 \mathrm{~d}-7.7 \mathrm{f}$ we observe a change in size of these pores. The pore size is affected by both the introduced artifacts and the algorithmically chosen threshold in these reconstructions.

Biologically interesting phenomena like emphysematic lung diseases introduce much larger defects in the lung tissue, where the size of the acinus is enlarged and the peripheral airways are collapsed [10]. Defects like these would still easily be detectable with an undersampled scan since the introduced artefacts are orders of magnitude smaller than the tissue alterations to be detected.

If other samples are to be observed using the proposed WF-SRXTM method, the desired level of image quality and therefore the corresponding reduction of the scanning time has to be defined according to the smallest structure present in the sample to detect.

The shorter scanning time obviously introduces minor artifacts in the reconstructed images but it is sometime desirable, especially when radiation sensitive samples need to be investigated. With a suitable protocol the dose can be reduced by $84 \%$ (Table 7.1), which might be a significant step towards tomographic imaging of sensitive samples using ultra high resolution and enhanced field of view. 
The field of view was increased three-fold by merging projections from three partially overlapping scans and reconstructing these resulting projections using the standard workflow at the TOMCAT beamline (Figure 7.4). The high precision of the linear motors used to move the sample stage (resolution better than $1 \mu \mathrm{m}$ in all three space directions, $0.1 \mu \mathrm{m}$ accuracy perpendicular to the beam direction [38]) permitted an highly reproducible positioning of the lung sample for the consecutive scans.

The sample rotation stage of TOMCAT has a run-out error of less than $1 \mu \mathrm{m}$ at $100 \mathrm{~mm}$ from the rotation surface [38]. This precise angular positioning made it possible to merge the projections from the consecutive subscans recorded at the same angular step but differing lateral position into one projection spanning the large field of view.

As a consequence of the sampling theorem, an increased amount of projections had to be acquired for an increase in the field of view, thus increasing the acquisition time. To overcome this limitation, we defined multiple scanning protocols with a reduced amount of total projections and thus reduced acquisition time and delivered dose (Table 7.1). All of these protocols were evaluated for the quality of the resulting reconstructions and compared to a gold standard scan. We have shown that the resulting quality can be simulated prior to scanning and thus provide a tool to choose a suited scanning protocol, based on the demands for scanning time optimization and quality of the resulting tomographic dataset (Figure 7.6).

Reducing the amount of projections for the central of the three subscans may be performed with a minor loss of fidelity in the resulting reconstructions. Let us compare protocols D/E and H/I. For protocols $\mathrm{E}$ and I we acquired half the amount of projections for the central subscan $s_{2}$ as compared to protocols $\mathrm{D}$ and $\mathrm{H}$. In both cases we reduce the scanning time by $17 \%$, but keep the quality of the scan on a comparable level (D: $70 \% \pm 3.09$ vs. E: $80 \% \pm 3.01$, H: $60 \% \pm 8.08$ vs. I: $56 \% \pm 3.23$ ). We show that the interpolation of missing projections does not introduce relevant errors in the resulting tomographic datasets.

For protocols with an equal amount of total projections, but differing amount of projections for the individual subscans (C/D and M/N) we observed minor differences in reconstruction quality. The qualities $\mathrm{E}_{\mathrm{i}_{\text {norm }}}$ of protocols $\mathrm{C}$ and $\mathrm{D}$ lie within their respective standard deviation ( $74 \% \pm 6.81$ vs. $70 \% \pm 3.09)$, and the qualities of protocols $\mathrm{M}$ and $\mathrm{N}$ are comparable $(52 \% \pm 4.71$ vs. $42 \% \pm 4.78)$. Both protocols $\mathrm{C}$ and $\mathrm{M}$ are scanned without oversampling the central subscan, making interpolation necessary, for protocols D and $\mathrm{N}$ we simply stitched the projections of the three subscans. Note that for protocol $\mathrm{N}$ we do undersample the outer parts of the sample. When deciding between two protocols with the same amount of total projections, it is thus desirable to favor the protocol where the central scan is not oversampled (i.e. choosing protocol $\mathrm{C}$ instead of $\mathrm{D}$ ). Even if this introduces additional computing time to interpolate projections prior to reconstruction, these protocols show an increased quality compared to protocols where the central scan is oversampled. Since an oversampling of the central scan does not add much to the total reconstruction quality and the outer parts of the sample contribute more to the total area of the projections, choosing a protocol where the sampling theorem is satisfied better for 
those parts of the sample is favorable (i.e. favouring protocol $\mathrm{M}$ to protocol N).

With the defined protocols we open the possibility for the end-user to choose an acquisition mode suited to fulfill the constraints on number of samples to be scanned within the allocated beamtime and desired quality of the reconstructed datasets.

Additionally, two special use-cases for different protocols are worth mentioning. First, if the user needs a very quick overview over samples at high resolution, a time-saving protocol can be used. This is especially the case, if the integrity of the sample can only be judged with a tomographic scan. Based on the quick scan the right samples for high resolution scans may be selected. It has to be mentioned that a quick overview could-in principle-be obtained with a lowresolution scan, which usually automatically accommodates a larger field of view. However, the resolution of such an overview scan is not always sufficient to detect interesting features in the samples which might be damaged.

We have shown that the field of view of parallel beam tomographic end-stations can be increased up to five-fold and have routinely reconstructed multiple tomograms with a three-fold increase in field of view. The shown acquisition protocols are theoretically expandable for more than five subscans, although the reconstruction of wide field scans with seven or more subscans would require an extremely powerful data processing infrastructure. The datasets shown in Figure $7 \cdot 7$ are binned scans resulting in datasets of 1024 slices, each with a size of $2792 \times 2792$ pixels at 8 bit gray value depth, which adds up to a total size of the dataset of approximately 7.5 GB. If we assume an unbinned scan with seven overlapping subscans, the size of one stitched projection will be approximately $14000 \times 14000$ pixels. The full dataset will consist of 2048 such slices, which would add up to a total size for the full dataset of approximately 383 GB.

Even if the amount of data to handle is huge, a wide field scan with a five-fold increase in field of view remains interesting, since it would enable the end-user to selectively reconstruct only regions of interest from large samples with ultra high resolution. Up to now, a two-step process was required to scan precisely defined regions from samples larger than the field of view. This process involved the use of different magnifications, two separate beamtimes and a precise registration of the samples between those beamtimes.

\subsection{SUMMARY}

A method to increase the lateral field of view of tomographic imaging has been established, which enables the high-resolution tomographic imaging of large samples that are wider than the field of view of the optical setup in multiple semi-automatically combined steps. Tomographic datasets of entire rat lung acini have been acquired with an enhanced field of view using WF-SRXTM.

Different optimized scanning protocols for covering a large field of view have been validated and are now provided for the end-users of the TOMCAT beamline. End-users now have the possibility to choose suitable scanning protocols depending on a balance between acquisition time and expected reconstruction quality. Depending on this balance, a reduction of the image acquisition time by $84 \%$ is possible, 
while keeping the quality of the reconstructed tomographic dataset on a level still permitting automated segmentation of the lung structure and surrounding airspace, as shown in subsection 7.4.3. The reduction in acquisition time obviously reduces the time during which the sample is irradiated by synchrotron radiation and thus reduces the radiation dose inflicted on the sample.

\subsection{ACKNOWLEDGMENTS}

This work has been funded by the grants 3100Ao-109874 and 310030125397 of the Swiss National Science Foundation. We thank Mohammed Ouanella for his excellent technical assistance and Volker Dicken from Fraunhofer MEVIS for the fruitful discussion concerning the euclidean distance transformation. Dan Ward kindly checked the English of the manuscript. 

Part III

DISCUSSION \& OUTLOOK 

And as soon as I'm done with these waffles, I shall discuss my evil plan!

— Invader Zim [133]: Episode 25A

The application of tomographic microscopy permits an unprecedented insight into the mammalian lung. It enables us to see and observe the object of interest to gain insight into the details of the sample to be studied. Besides the vivid images of the beautiful biological samples which spur the interest in this line of work, biological questions can be scientifically answered using tomographic images as a basis.

The tomographic data obtained at the beamline for TOmographic Microscopy and Coherent rAdiology experimenTs (TOMCAT) offers a three-dimensional insight into the mammalian lung with extremely small voxel sizes down to $370 \mathrm{~nm}$. High resolution images of the terminal airways can also be obtained with Scanning Electron Microscopy (SEM) (see Figure 8.1 and [10, Figure 12]) but these images only provide a view of the surface structure of a sample and are not suitable for a full three-dimensional reconstruction of the terminal airways. Using SEM or Transmission Electron Microscopy (TEM) it is impossible to visualize the larger three-dimensional structure of the terminal airways inside the lung since the sample has to be cut prior to acquiring images using this method, which essentially destroys the full threedimensional information of the sample. Using Electron Microscopy (EM) tomography, ultra high three-dimensional information from a sample can be obtained, but the available sample volumes of maximally $64 \mu \mathrm{m}^{3}$ [134] and generally around $1 \mu \mathrm{m}^{3}$ [135] are too small for the analysis of the terminal airway structure and single rat lung acini which are around $1.9 \mathrm{~mm}^{3}$ [28].

In contrast to TEM and SEM, Computed Tomography (CT) offers a nondestructive insight into the lung Relatively low resolution micro-Computed Tomography $(\mu \mathrm{CT})$ enables the study of the major airways of swine [79], rat [136, 137] or mice lung [115, 138]. Ultrahigh resolution Synchrotron Radiation based X-ray Tomographic Microscopy (SRXTM) enables the study of the functional units of the pulmonary gas exchange area, the so-called acini with unmatched resolution and precision [19, 139]. 


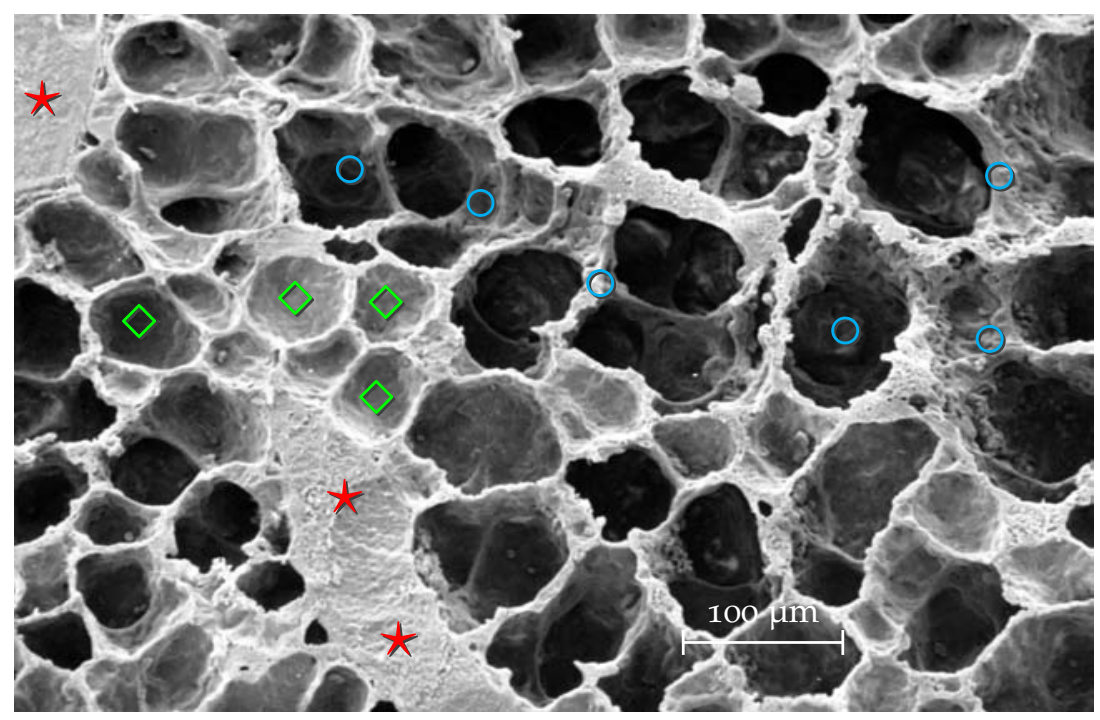

Figure 8.1: SEM image of a rat lung sample obtained at postnatal day 8. Single alveoli are clearly visible (a few representative alveoli are marked with green diamonds $(\diamond))$, but the full three-dimensional information cannot be extracted from this type of images. The red stars $(\star)$ mark a larger blood vessel, probably a vein, the cyan circles $(\bigcirc)$ mark two alveolar ducts. Image from the EM image library of the Institute of Anatomy.

In this work, three imaging methods based on ultra high resolution tomographic data of the terminal airway were used to answer biological questions. chapter 5 and chapter 6 represent minor parts, chapter 7 represents the major part of the work done for this thesis. The following sections discuss the notable points of each chapter, their respective lengths represent the importance of their corresponding chapters for this thesis.

\subsection{MULTimOdAL IMAGING}

The method presented in chapter 5 combines the advantages of tomographic and electron microscopy imaging into a multimodal imaging approach for the analysis of inhaled particles in the lung. The tomographic data provides the unrestricted three-dimensional information on the location of sub-micron particles in the terminal airway tree which is combined with high resolution EM images for the precise analysis of those particles in the lung tissue.

It is expected that inhaled particles are deposited in specific locations in the lung (personal communication Prof. Dr. Johannes C. Schittny and Prof. Dr. Wolfgang Kreyling). Currently available methods cannot easily determine the exact location of these deposition sites in relation to the airways tree. The presented multimodal imaging approach overcomes this limitation.

Classic histological sections analyzed using light and electron microscopy allow for a precise localization of the inhaled particles in relation to the tissue, i.e. make it possible to analyze the interaction of the particles with alveolar macrophages and epithelial cells [140]. But the precise localization of these interaction sites inside the airway tree are virtually impossible to extract using histological sections since the 
three-dimensional structure of the sample is destroyed.

The registration of such classic histological sections with the threedimensional data obtained through SRXTM makes it possible to localize sites of interaction along the airway tree inside the three-dimensional structure of the terminal airways. The method presented in chapter 5 was based on careful alignment of the sample prior to physical sectioning. This made registration between the two-dimensional EM image and the three-dimensional tomographic dataset straightforward. The images only needed to be corrected for rotation around the z-axis and translation to correlate between a physical EM slice and a virtual SRXTM slice. Currently, the alignment of the alignment of the scanned rat lung sample prior to histological sectioning is the most crucial step in the presented workflow.

Figure 8.2 shows three-dimensional visualizations of different alignments between the two multimodal datasets. The situation shown in Figure 8.2a can be achieved through careful alignment of the sample prior to histological sectioning and corresponds to the currently available method. The fully unrestricted three-dimensional information of the airway tree from the tomographic dataset can be matched with two-dimensional information about the particle location in or around the tissue in the lung from the EM image.

A working registration method for two multimodal datasets oriented with one degree of freedom in respect to each other (as shown in Figure $8.2 \mathrm{~b}$ ) would decrease the need for extremely precise alignment prior to sectioning of the sample. Increasing the robustness of the registration between the datasets to multiple degrees of freedom (as shown in Figure 8.2c) would allow for arbitrary orientation of the sample between the two imaging modalities and thus increase the sample throughput.

Current work in our group focuses on an automatic registration method taking into account all six degrees of freedom i.e. rotation around the $\mathrm{x}, \mathrm{y}$ and $\mathrm{z}$-axis and translation in the $\mathrm{x}, \mathrm{y}$ and $\mathrm{z}$-plane. First results have already been presented as a Master thesis in our group [142] and will be pursued as part of a Ph.D.-thesis.

\subsection{FINITE ELEMENT RECONSTRUCTION OF THE TERMINAL AIR- WAYS}

The results presented in chapter 6 focus more on the functional analysis of the gas-exchange region of the lung. The alveolar structure of the pulmonary acinus plays a vital role for the gas exchange function. Using an analytical method which mapped a Finite Element (FE) representation of the terminal airways over a three-dimensional reconstruction of an SRXTM dataset we accurately reconstructed the gas exchange regions of the lung in three dimensions with a resolution of $1.48 \mu \mathrm{m}$ per voxel.

To our knowledge the structure-function relationship of the parenchymal region of the lung has never before been analyzed in such high resolution in three dimensions. Berend et al. [77] performed an analysis of the peripheral region of one human lung using serial sections studied by light microscopy. They were able to analyze the structure of one partial acinus through formidable effort and stated that the process proved to be very difficult and time consuming. Litzlbauer et al. [79] analyzed porcine lungs using $\mu \mathrm{CT}$ but suggest to favor SRXTM over 


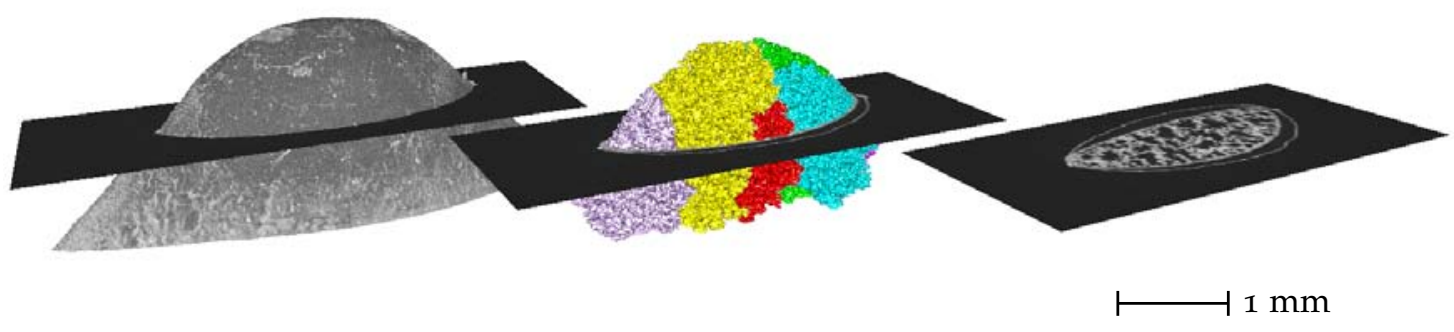

(a) Planar orientation of slice

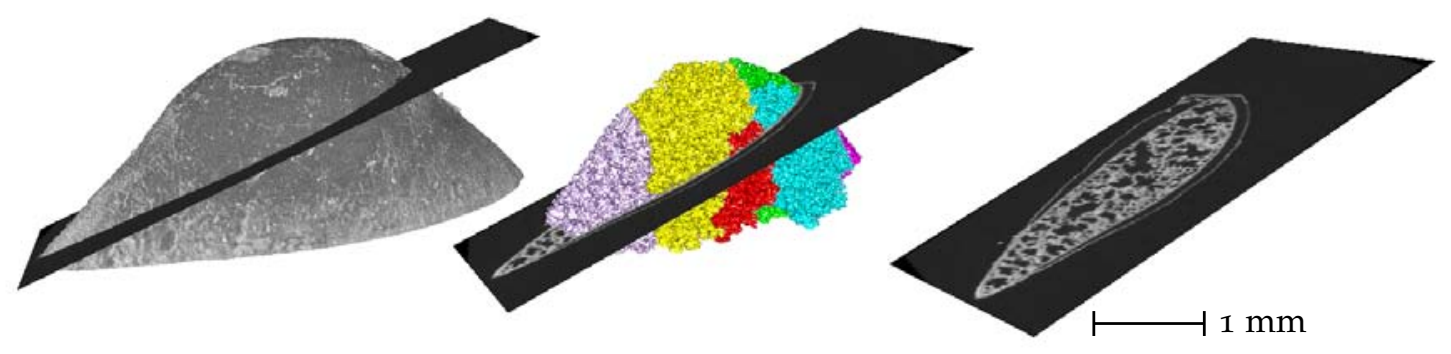

(b) Arbitrary orientation of slice, one degree of freedom

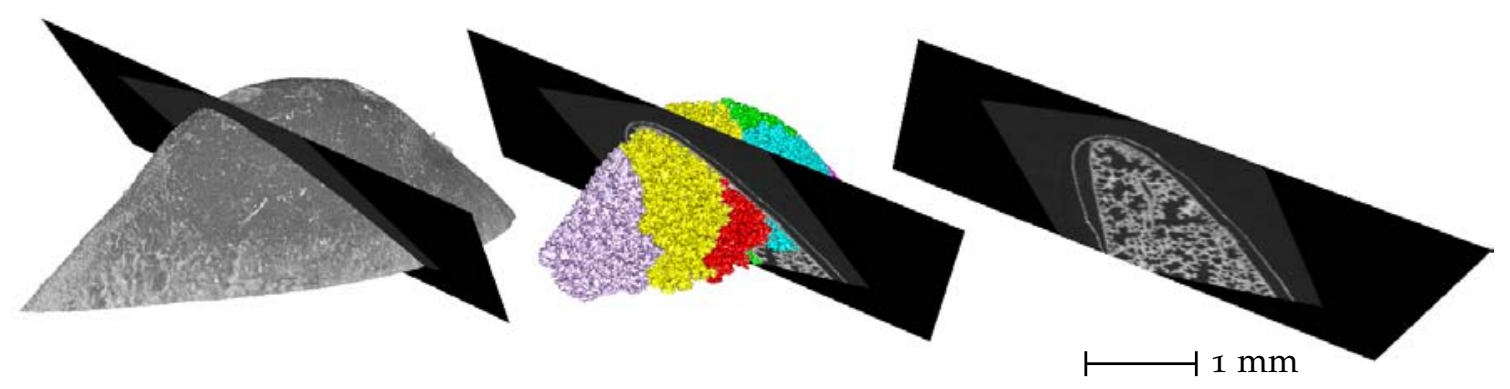

(c) Arbitrary orientation of slice, multiple degrees of freedom

Figure 8.2: Multimodal Imaging: Alignment of datasets from two imaging modalities. Left: Sample from SRXTM with overlaid twodimensional image. Center: Five independent airway segments have been extracted and are three-dimensionally visualized. Right: Two-dimensional slice shown with three-dimensional orientation in relation to the SRXTM dataset. (a): Planar orientation of the slice obtained with the second imaging modality. Through careful orientation of the sample prior to histological cutting, a registration is straightforward, since only the rotation in the plane of the slice and the different magnification have to be taken into account. (c): Pitch-angle [141] rotation between the two datasets. (c): Pitch and roll rotation between the two datasets. Note that-for the sake of illustration-the shown slice is from the original tomographic dataset and not from a light or electron microscopy slice, as described by [142]. 
$\mu \mathrm{CT}$ to enhance the quality of morphometric analysis of the terminal airway structure.

With the FE meshing of the reconstructed datasets we were not only able to visualize lung tissue and air spaces of the alveolar region in rat lung samples in three dimensions, but also prepared the reconstructions for further analysis of the terminal airway region. The assessment of different morphological parameters like volume density of tissue and surface area density of air space, two well characterized stereological parameters was carried out on the three-dimensional reconstructions. The three-dimensional data permitted the refining of the region of interest for the analysis, making it possible to easily isolate multiple alveolar ducts, something which would not have been possible using classic histological sections.

The FE meshing also prepared the resulting dataset for Computational Fluid Dynamics (CFD) analysis of the airflow in the lung. Several collaborators used the extracted datasets for simulating the airflow $[143,144]$ and particle deposition [145] in the terminal airways.

\subsection{Wide Field Synchrotron Radiation Based X-ray To- MOGRAPHIC MICROSCOPY}

The wide field scanning method presented in chapter 7 represents the main results achieved in this thesis. This newly developed Wide Field Synchrotron Radiation Based X-ray Tomographic Microscopy (WF-SRXTM) method makes it possible to obtain high resolution tomographic data of larger lung samples than with conventional SRXTM. Prior to the presented WF-SRXTM scanning method, obtaining tomographic datasets of entire acini required sophisticated and time consuming centering and alignment techniques. With the increase in the field of view we can routinely record tomographic images of full acini in the terminal airways.

This new method makes enables to study the changes in the acini over the lung development. Using classic image processing methods like segmentation of airspaces and calculating their volume we can study acinar growth and changes in the distribution of alveolar size, as show in Figure 7.9. These parameters are of great biological interest, since changes in the configuration of the terminal airways occurring through changes in acinar size influence airflow as well as particle deposition in the lung. The growth behavior of the alveoli over postnatal lung development can be studied at different locations in the lung, e. g. close to the pleural region or deep inside the lung, close to the larger conducting airways.

The following section focuses on some biological results achieved with WF-SRXTM instead of an additional discussion of the technical details of the method.

We routinely performed more than 70 WF-SRXTM scans of different rat lung samples. Multiple airway segments inside these tomographic datasets have been extracted with a region growing algorithm [129, 146] and the structure of these airway segments has been visualized using a skeletonization algorithm built into MeVisLab [147], the development environment for medical image processing and visualization used almost exclusively for the analysis and visualization of the tomographic datasets in the present work. 


\subsubsection{Skeletonization}

In the three-dimensional Euclidean space, the skeleton or the medial surface of any structure corresponds to the location of the centers of all the maximal inscribed spheres [148]. The skeleton representation of an arbitrary structure includes all information about the original shape of the object and, at the same time, facilitates an algorithmic geometrical and structural shape analysis [149].

Figure 8.3 visualizes the whole process in a two-dimensional example. An in-depth explanation of the skeletonization algorithm built into MeVisLab is presented by Selle et al. [149], an application of the algorithm for the morphometric quantification of vessels is presented by Boskamp et al. [150].

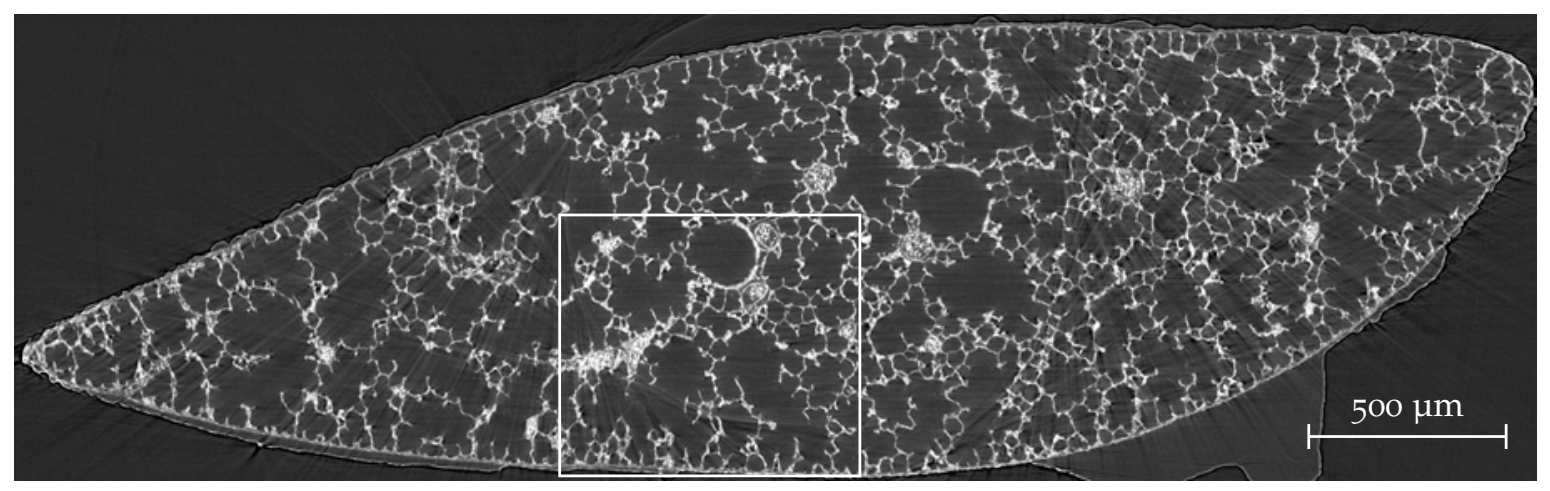

(a) Original slice

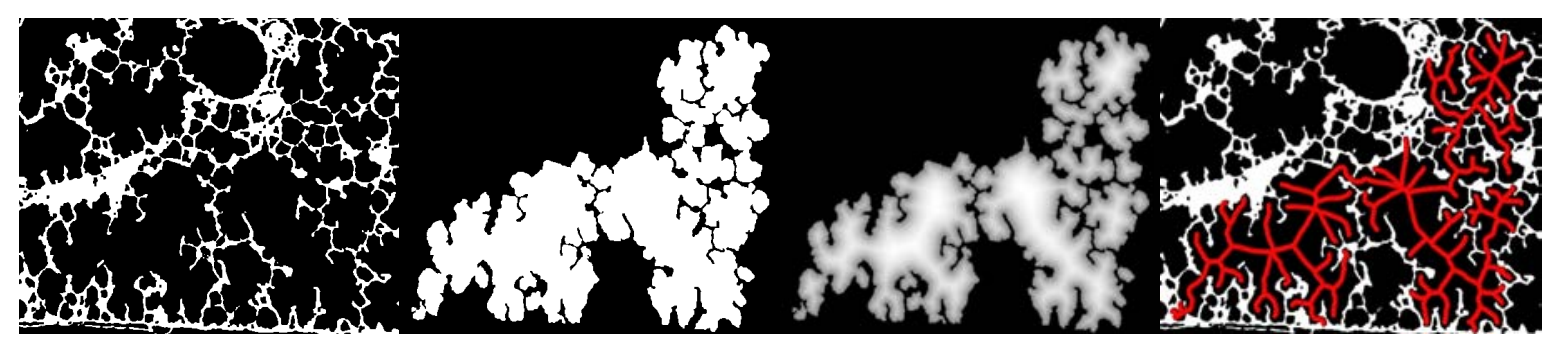

(b) Binarized

(c) Segmented

(d) Distance Transformation

(e) Skeleton

Figure 8.3: Two-dimensional skeletonization process: (a): Wide field scanned tomographic slice from a rat lung sample obtained at postnatal day 21. The inset corresponds to the region of the images shown in the bottom row. (b): Binarized ROI The data has been separated into tissue and airspace. (c): The white segment represents one connected airway structure inside the ROI in two dimensions. (d): Euclidean distance transformation of the segment from panel (c). The gray level value of each pixel represents the distance to the border between tissue and airspace. (e): The local maxima of the distance transformation form the skeleton which is then overlaid on the binarized lung structure from panel (a).

MeVisLab can export the information of the skeleton to an XML-file from which we can obtain the amount of skeleton nodes and their position in space for further analysis. The example in Figure 8.4 shows a three-dimensional skeletonization of multiple acini in a rat lung sample obtained at postnatal day 21 containing multiple airway segments which each contain one or more acini. Both of these structures are shown in the background. The foreground of the image shows the 
skeletons of the extracted segment containing between 1100 and 7300 nodes.

Such exported skeletons are currently used in our group to analyze changes in the dimensions and branching pattern of the terminal airway segments over postnatal lung development e.g. introduction of new bifurcations and changes in the length of the terminal bronchioles. Future work aims to utilize the skeletonization information combined with the extraction of single acini in the tomographic datasets as described in chapter 9 .

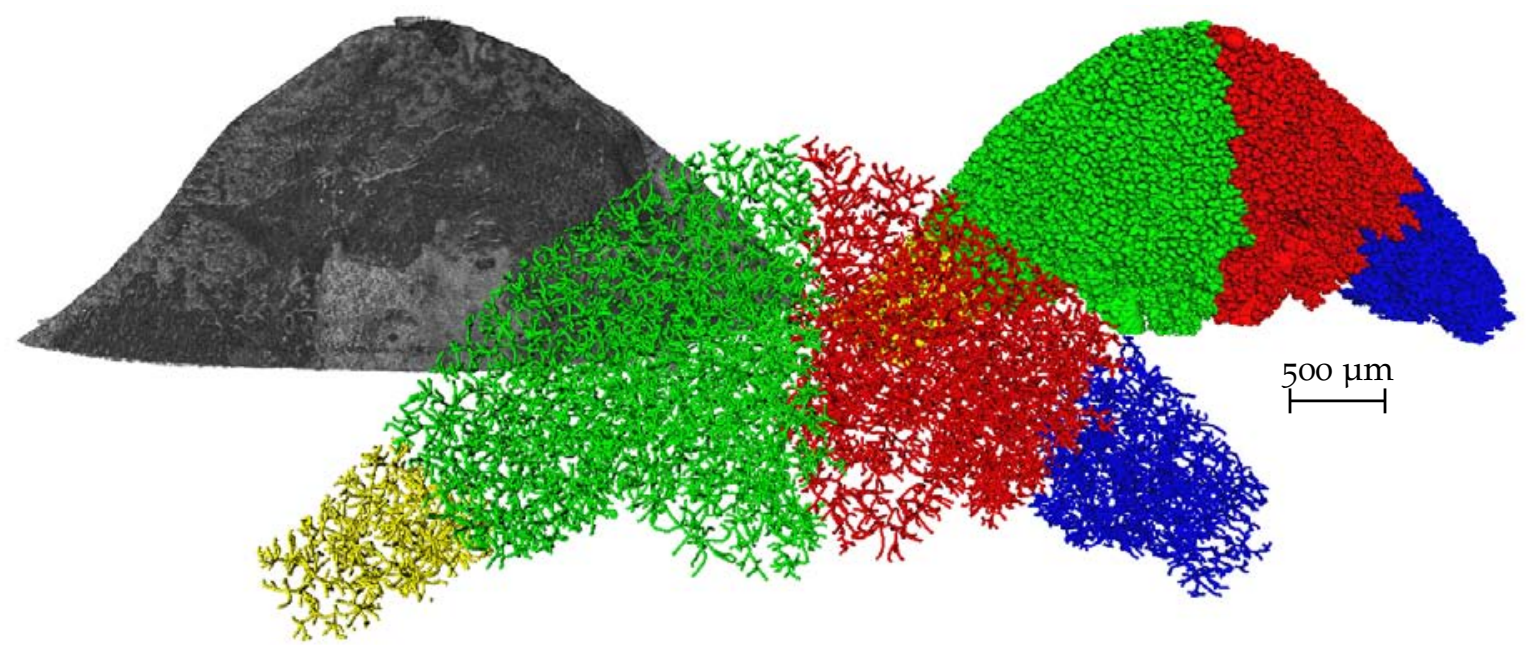

Figure 8.4: Three-dimensional visualization of rat lung sample obtained at postnatal day 21. Left: Three-dimensional view of the sample, Right: Four independent airway segments containing multiple acini. Foreground: Extracted airway skeletons of the independent airways. The yellow skeleton contains 1133 , the green 7288 , the red 6513 and the blue skeleton 3278 nodes.

Extracting the four large independent airway segments as shown in Figure 8.4 would not have been possible using classic SRXTM. Enhancing the field of view of the tomographic scans enabled the visualization of multiple acini in the lung and made more descriptive results possible; such results, namely a stereological analysis of single acini is presented in the following section.

\subsubsection{Stereological analysis of the tomographic data}

As mentioned in chapter 1 , the interrelation of the acini in the lung tissue cannot be analyzed by using classical sectioning methods, since the three-dimensional information of the airway structure is destroyed during the sectioning process. Applying a region growing method on tomographic datasets we were able to study this interrelation of the acini on virtual slices of a rat lung sample scan.

Multiple independent airway segments were extracted from the threedimensional WF-SRXTM datasets and were subdivided into single acini using a method named man hole covering. The entrances of the acini corresponding to the border between the purely conducting and the gasexchanging region were identified using morphological criteria like appearance of alveoli in the airway walls and so-called manhole covers have been manually drawn over the tomographic slices.

The method is called man hole covering because the manually drawn circles closing the airway lumen resemble a circular manhole cover. 
Figure 8.5 a shows a three-dimensional view of seven acini segmented using this manhole cover method. The red rings show the location of four manhole covers, the other covers are occluded by their respective acinus. Figure $8.5 \mathrm{~b}$ and $8.5 \mathrm{c}$ show slices from the original WF-SRXTM dataset combined with a two-dimensional view of the threedimensional segmentation shown in Figure 8.5a.

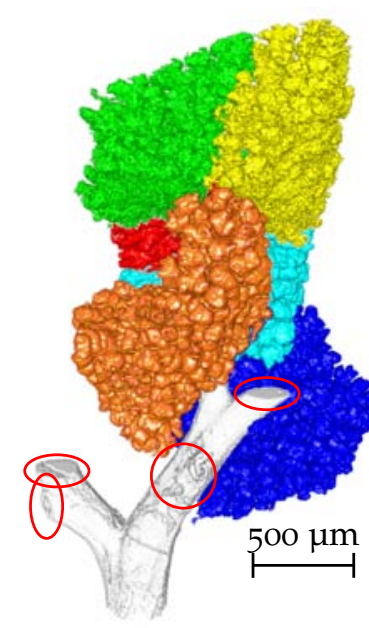

(a) Three-dimensional view

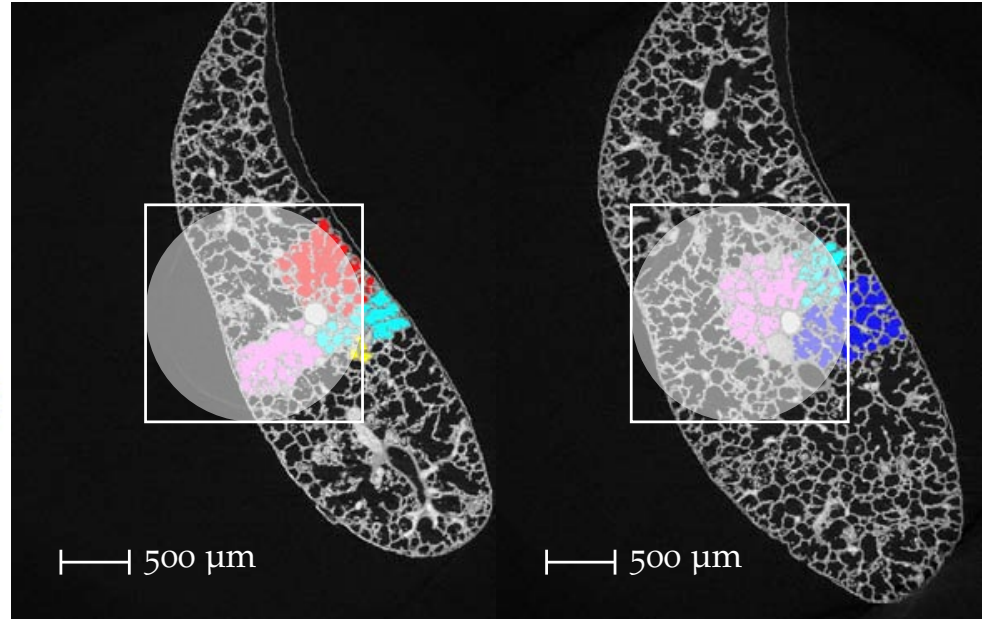

(b) Slice 804

(c) Slice 1168

Figure 8.5: Manhole cover process for a rat lung sample obtained at postnatal day 4: (a): Three-dimensional view of seven segmented acini (one not visible). The red rings mark the locations of manually drawn manhole covers, which are not shown for clarity. The covers used to segment the visible acini are hidden by the shown segments. (b) and (c): Slice 804 and 1168 of the WF-SRXTM dataset (black-and-white image), combined with the two-dimensional information on the location of the segmented acini (colored regions) shown in panel (a). Both slices are slightly cropped in horizontal direction. The white square marks the field of view and the semitransparent circle marks the usable region from a classic SRXTM scan at TOMCAT. The increase in field of view is immediately evident. The regions of the red, cyan, yellow and blue acinus outside the square would not have been detected using a classic scan, but are included in the WF-SRXTM dataset.

The white square in the middle of the images shows the field of view of a tomographic image obtained with classical tomography at TOMCAT. The semitransparent white circle marks the region of usable data from a scan with classic field of view. It is clearly visible-even if the sample does not completely fill the field of a WF-SRXTM scan and could have been bigger-that the usable volume of the tomographic dataset is greatly increased.

Combining the three-dimensional segmentation (colored regions) with the original tomographic slices (black-and-white image) makes it possible to assign alveoli on the two-dimensional slices to the three-dimensional location of the acini and to understand the interrelation of the acini even on the two-dimensional slices. Additionally, it enables the use of these combined images for subsequent stereological analysis.

For the analysis of multiple acini in our lung samples we not only needed to increase the field of view horizontal direction as presented in chapter 7 , but also in vertical direction. We thus performed a so- 
called stacked wide field scan, where multiple WF-SRXTM datasets are sequentially recorded on top of each other, in order to cover an increased field of view in both horizontal and vertical direction. The analysis presented below was performed on such stacked WF-SRXTM datasets with a volume of approximately $3000 \times 3000 \times 3000$ voxels at an isometric voxel size of $1.48 \mu \mathrm{m}$, i.e. a 27 -fold increase in visible sample volume as compared to a single classic tomographic scan.

Using WF-SRXTM slices of rat lung samples obtained at different postnatal days we assessed the number of alveoli per volume of the segmented acini. Using the aforementioned manhole cover method, several single acini have been three-dimensionally extracted and overlaid over the two-dimensional tomographic slices, as shown in Figure 8.5b and 8.5c. The volume of the extracted acini shown in Figure 8.5a has been calculated according to the amount of contained voxels.

Currently achieved results hint towards a larger change of the acinar size during lung development than previously assumed (Data not shown). There is a clear distinction between the conducting and the gas-exchanging airways (as described in section 2.2) for days four, 10 and 21, while for days 36 and 60 the change between those two points seems more gradual. Since the segmentation of single acini at day 36 and 60 is much more complicated than at the earlier time points, additional work is needed to clarify the results. Segmenting more acini on the already present datasets will help to describe this change in more detail.

\subsubsection{STEPanizer}

Tomographic data obtained at TOMCAT is inherently isotropic, i.e. the voxels are isometric cubes. This makes the tomographic data well suited for morphological analysis using a stereological approach, which can be used for the estimation of morphometric parameters of threedimensional objects based on measurements made on two-dimensional sections [151].

Using the STEPanizer [152, available online], the number of alveoli per acinar segment has been evaluated. The STEPanizer is a simple, web-based tool for manual stereological assessment of digital images developed by Dr. Stefan Tschanz at the Institute of Anatomy, University of Bern. The beta-version of the STEPanizer with enabled disector-which according to Hsia et al. [35] enables accurate measuring of alveolar number and volume-was used to count the appearance or disappearance of septal bridges in the tomographic datasets.

Counting these septal bridges correctly estimates the number of alveolar entrance rings which in terms corresponds to the number of alveoli in the volume. Details on the counting process have been described by Hyde et al. [153] and Ochs et al. [154].

Using the number of bridges and the volume of the alveolar segments, the number of alveoli per volume has been plotted as shown in Figure 8.6. A statistically significant increase of the alveolar number per volume is observed from day four until postnatal day 21. From day $21 \mathrm{on}$, the alveolar number per volume significantly decreases.

We were able to show that applying the optical disector [35, 155] to tomographic data yields results equal to results obtained from classic histological sections. On classic histological sections, the alveolar number has been assessed in relation to the total lung volume using a computer assisted stereological toolbox (newCAST, Visiopharm A/S,
Since tomographic images are inherently a stack of slices with a known distance to each other, adjacent slices can be used for disector-type analysis [35]. 


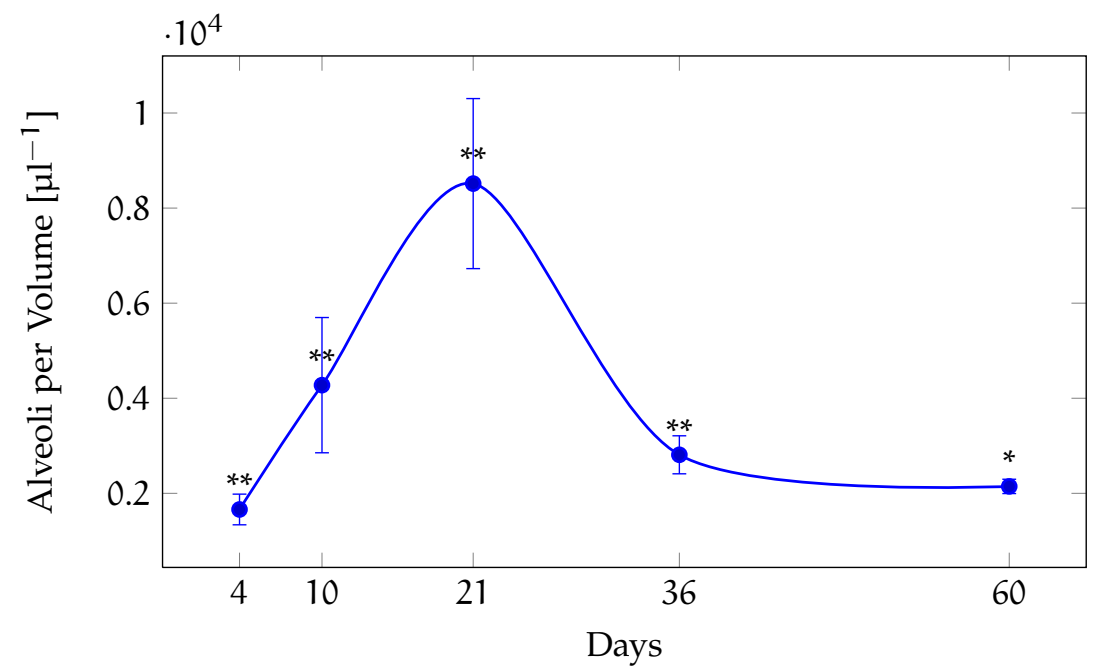

Figure 8.6: Alveolar entrance rings per volume. Multiple airway segments per day have been counted using the STEPanizer. Data shown at day 10 and 21 are averaged values from multiple rat lung samples, the other points are averaged values from one rat lung sample. Volumes of the segments have been evaluated using MeVisLab. The average of the alveoli (bridges) per volume for each day has been plotted. The error bars show the calculated standard deviation based on the entire population. The probability associated with a Student's $t$-Test $p$ calculated between the single days is $p_{4: 10}=0.0056$, $p_{10: 21}=0.0007, p_{21: 36}=0.0017$ and $p_{36: 60}=0.0358$. ${ }^{* *}$ denotes a $\mathrm{p}$-value $\mathrm{p}<0.01{ }^{*}$ denotes $\mathrm{p}<0.05$.

Hoersholm, Denmark, Data not shown). For the tomographic datasets, alveolar number has been assessed for several single acini with the STEPanizer and are shown in Figure 8.6. The obtained results from tomographic data and classic histological slices lie within their respective standard deviation (Personal communication Lilian Salm).

Performing this stereological evaluation on tomographic data offers an increased insight into the structure of the lung. A correct assignment of the different alveoli to their corresponding acinus is not possible using classic histological sections. In contrast, a three-dimensional region growing segmentation of the acini on tomographic data makes this task easily feasible. 
The tomographic images used in this work served as a ground truth to obtain insights into the mammalian lung. Morphometric parameters of the terminal airways obtained on three-dimensional reconstructions of tomographic data have been used to examine structural and functional parameters of the gas-exchange region in rat lung samples.

Wide field scanning has become a routine scanning method in our group. To make this method available for other researchers, several improvements are planned for the future. The current wide field scanning workflow uses custom MATLAB-scripts to merge the projections from the several recorded subscans. The goal for the next few months will be the integration of the present wide field scanning workflow into the image processing pipeline of TOMCAT, especially into the software used to convert the projections to sinograms, called Prj2Sin. Prj2Sin already integrates the standard $360^{\circ}$ off-center sample rotation method to double the horizontal field of view (as shown in Figure 7.1b). For such a $360^{\circ}$ scan all projections are acquired in one consecutive sequence, and through simple resorting of the projections on the disk Prj2Sin can compute the overlap between the images and calculate the merged sinograms. For a Wide Field Synchrotron Radiation Based X-ray Tomographic Microscopy (WF-SRXTM) scan we generally acquire three separate subscans in three different directories. At the moment, the merging of one dataset with totally 10734 projections from three subscans takes more than one hour to perform using the MATLABscripts. The process involves the detection of the overlap between the individual subscans, reading and merging the original projections and writing these merged projections to disk prior to the generation of merged sinograms. Stitching the projections directly with Prj2Sin should reduce the time used for processing the acquired projections to merged sinograms to reconstructed tomographic wide field scan slices. Integrating the workflow into the image processing pipeline would both decrease the merging time and make the whole process available to other users of the beamline as an easy to use software package. 
The feasibility of the enlargement of the horizontal field of view of tomographic endstations has been demonstrated and the resulting datasets have been used for the analysis of large volumes of the terminal airways with ultra high resolution. Future work will focus on the use of WF-SRXTM datasets for the extraction and analysis of the acini in the lung, improving the preliminary results presented in subsection 8.3.2.

The presented method which uses so-called manhole covers to separate single acini from a central airway has been applied to multiple rat lung datasets recorded over the course of the postnatal development from day four to day 6o. The definition of the location of the manhole covers in the airway tree is a process relying on manually locating the position of the junction in the airway based on morphological criteria and subsequent drawing of the manhole cover at these locations. Combining the aforementioned manhole cover method with a skeletonization of the airway tree to act as a guideline for the positioning of the manhole covers would make it possible to extract and segment large numbers of acini for further analysis in relatively short time.

Additionally, we plan to use the obtained WF-SRXTM datasets for the extraction of larger segments of the terminal airways than currently available in order to improve existing models of airflow inside the terminal airways using Computational Fluid Dynamics (CFD).

We plan to stereologically analyze the tomographic datasets in more detail using the STEPanizer. With the first results presented in chapter 8 we have shown that-as expected-analyzing tomographic data yields results which match stereological results obtained from classic histological sections. Performing the stereological analysis on virtual tomographic slices is advantageous compared to performing the analysis on real histological slices, since tomographic data can be recorded without destroying the sample and gives a fully unrestricted threedimensional view inside the sample in addition to the stereological assessment. 
Part IV

BACK MATTER 

This part of the thesis contains a Curriculum Vitæ with a list of selected publications, the Bibliography and other necessities to fulfill the guidelines of the Graduate School for Cellular and Biomedical Sciences. 


\section{David Haberthür}

09.2006-present Ph. D.-Student, Institute of Anatomy, University of Bern, Bern, Switzerland.

Ultra-High resolution lung imaging to study lung development. Synchrotron radiation based tomographic microscopy images were used to visualize the three-dimensional lung structure at high resolution and to study the lung development of mice, rat and primates.

10.2006-08.2008 Master of Advanced Studies ETH in Medical Physics, Swiss Federal Institute of Technology, Department of Physics, Zürich, Switzerland.

Extra occupational Master of Advanced Studies ETH in Medical Physics, a two-year program designed for M.Sc. or Ph. D.-Students. Acquired fundamental knowledge in medical physics, and specialized in different fields of medical physics, e.g. biocompatible Materials, Radiotherapy, medical Optics and Acoustics.

05.2001-10.2002 Master Student, Biomedical Photonics, Institute of applied Physics, University of Bern, Bern, Switzerland.

Master Thesis: Use of Lasers in Medicine: Tissue Soldering and Precise Cutting. Studied laser applications in medicine, including interaction of lasers with cartilage and heart tissue. Performed study on tissue soldering.

01.2000-07.2000 Exchange Student, Department of Physics, Strathclyde University, Glasgow, Scotland, United Kingdom.

Exchange term in the United Kingdom, multiple lectures including Software Design, Imaging and Laser physics.

\section{Theses}

Ph. D.-Thesis High resolution tomographic imaging of the alveolar region of the mammalian lung - A close look deep into the lung, Supervisors: Prof. Dr. Johannes C. Schittny and Dr. Mauricio Reyes.

I am currently studying lung development using three-dimensional datasets obtained with high resolution synchrotron radiation based $x$-ray tomographic microscopy. I have developed a skeletonization work flow at our group using the image processing software MeVisLab using multiple visualization pipelines and pre-existing modules and strive to extract quantitative information from the branching pattern in the gas-exchange region of the mammalian lung.

Post-graduate Master Thesis
Quality guided wide field x-ray tomographic imaging, Supervisors: Dr. Christoph Hintermüller and Prof. Dr. Marco F.M. Stampanoni.

During this master thesis I implemented the necessary acquisition protocols at the TOMCAT beam line of the Swiss Light Source to increase the field of view of the tomographic imaging process in horizontal direction. This implementation is the base for imaging of samples bigger than the size currently possible, while keeping the resolution at the desired level. Different image acquisition protocols have been implemented for providing the end-user of the beam line the possibility to acquire quality guided tomographic wide field scans of his samples in an unattended, automatic way. 
Master Thesis Use of Lasers in Medicine: Tissue Soldering and Precise Cutting, Supervisor: Prof. Dr. Martin Frenz.

During my master thesis I worked with infrared diode-lasers to coagulate a dye-enhanced solder to join cartilage tissue. The goal of the study was to evaluate the influence of laser parameters and solder ingredients on the tensile strength and the thermally induced cartilage damage of a bond between cartilage and an implant. Additionally I worked with a pulsed Er:YAG laser for ablation and cutting of calcified heart tissue.

\section{Skills}

Academic Objective

Since the start of my academic career I have been fascinated by the combination of physics, imaging and medical research. During my Ph. D.-thesis I have been working on new imaging methods for the study of the three-dimensional lung structure during development. The fractal structure of the lung is eerily fascinating on all levels, I strive to provide possibilities to assess this structure on a minute level-down to the single alveoli. To support the ongoing effort to study lung samples in their full size, I have developed a method of enhancing the field of view of tomographic imaging as part of my master thesis of the extra-occupational master of advanced studies.

Visualization MeVisLab, Imaris, Amira

Analysis of tomographic datasets is computationally intensive. I have developed several work flows using MeVisLab, Imaris and Amira for the three-dimensional visualization and analysis of tomographic datasets of mammalian lungs, several GB in size.

OS Windows, Mac OS X, Linux

Very good user and good administration experience.

Programming MATLAB, Python

I have developed a lot of programs using MATLAB and Python, be it for the processing of multi-GB datasets or the interaction with the TOMCAT-beam line at the Swiss Light Source.

Publishing LATEX, Internet, Microsoft Office

Nearly all my documents are prepared in LTEX, Manuals for our group are shared on my work wiki website and I have a very good grasp of all Microsoft Office programs.

\section{Languages}

German Mother Tongue

English Very Good

French Fluent

Highly experienced in spoken and written English

Italian Fluent

High-school level

As a kid I lived in Italy for 6 years

\section{Other Interests}

Photography I really enjoy digital photography as a whole, but especially like technical challenges like panorama photography.

Sports Be it a solitary sport like swimming (since about 25 years), biking (I used to work as a bike messenger) and skiing or team sports like ultimate frisbee, I'm up for it!

Cooking I am looking forward to the day I have cooked all the recipes in my cookbooks at home. 
Nenad Filipovic, David Haberthur, Frank S. Henry, Danko Milasinovic, Dalibor Nikolic, Johannes C. Schittny, and Akira Tsuda. Recirculation Identified In A 3D Alveolar Duct Reconstructed Using Synchrotron Radiation Based X-ray Tomographic Microscopy. Am. J. Respir. Crit. Care Med., 181(1-MeetingAbstracts):A2192-, 2010.

David Haberthür. Quality guided wide field x-ray tomographic imaging. Master's thesis, University of Bern, Switzerland, Paul Scherrer Institut, Switzerland, ETH Zürich, Switzerland, August 2008.

David Haberthür, Christoph Hintermüller, Johannes C. Schittny, and Marco Stampanoni. Quality Guided Synchrotron Radiation Based X-Ray Tomographic Microscopy of Large Lung Samples. Am. J. Respir. Crit. Care Med., 179(1-MeetingAbstracts):A1060-, 2009.

David Haberthür, Christoph Hintermüller, Akira Tsuda, Marco Stampanoni, and Johannes C. Schittny. Generation of Acinar Skeletons after Synchrotron Radiation Based X-Ray Tomographic Microscopy of the Lung Parenchyma. Am. J. Respir. Crit. Care Med., 179(1-MeetingAbstracts):A3531-, 2009.

David Haberthür, Manuela Semmler-Behnke, Shinji Takenaka, Wolfgang G. Kreyling, Marco Stampanoni, Akira Tsuda, and Johannes C. Schittny. Multimodal imaging for the detection of sub-micron particles in the gas-exchange region of the mammalian lung. volume 186 of Conference Series, page 012040 (3pp). Journal of Physics, IOP Publishing, 2009.

David Haberthür, Christoph Hintermüller, Federica Marone, Johannes C. Schittny, and Marco Stampanoni. Radiation dose optimized lateral expansion of the field of view in synchrotron radiation x-ray tomographic microscopy. Journal of Synchrotron Radiation, 2010. in Press.

M. Sausbier, C. Dullin, C. Kabagema, K. Flockerzie, D. Haberthür, J. Wessels, F. Alves, J. C. Schittny, W. Neuhuber, P. Ruth, and U. Sausbier. Enhanced cathepsin k release from osteoclasts is linked to idiopathic osteoporosis in mice with BK channel ablation. Naunyn-Schmiedeberg's Archives of Pharmacology, 379(Suppl. 1):147, APR 2009. 50th Annual Meeting of the Deutsche-Gesellschaft-fur-Experimentelle-undKlinische-Pharmakologie-und -Toxikologie, Mainz, GERMANY, MAR 10-12, 2009.

Ulrike Sausbier, Christian Dullin, Clement Kabagema, Katarina Flockerzie, David Haberthür, Marco Stampanoni, Johannes C. Schittny, Winfried Neuhuber, Walter Stühmer, Peter Ruth, Frauke Alves, and Matthias Sausbier. BK channel ablation in osteoclasts leads to enhanced Cathepsin $\mathrm{K}$ release and is linked to idiopathic osteoporosis in mice. Nature Medicine. submitted.

Johannes C. Schittny, David Haberthür, Manuela Semmler-Behnke, Shinji Takenaka, Marco Stampanoni, Wolfgang G. Kreyling, and Akira Tsuda. High Resolution 3dimensional Imaging Of Ultrafine Particles In The Lung Parenchyma. Am. J. Respir. Crit. Care Med., 181(1-MeetingAbstracts):A3643-, 2010.

Akira Tsuda, Nenad Filipovic, David Haberthür, Renee Dickie, Yasuto Matsui, Marco Stampanoni, and Johannes C. Schittny. Finite element 3D reconstruction of the pulmonary acinus imaged by synchrotron X-ray tomography. J Appl Physiol, 105(3):964-976, 2008. 
[1] Wikipedia. You'll Never Walk Alone (song) - Wikipedia, The Free Encyclopedia, 2010. URL http://en.wikipedia.org/w/ index . php?title=Young) \&oldid=360900902. [Online; accessed 11-May-2010]. (Cited on page xiii.)

[2] Bill Watterson. It's A Magical World: A Calvin and Hobbes Collection. Andrews McMeel Publishing, original edition, 91996. ISBN 9780836221367. URL http://books.google.com/books? id=wmjG5g4Yx7gC\&lpg=PA4\&pg=PA481. (Cited on page 3.)

[3] Kenneth H. Downing, Haixin Sui, Auer, and Manfred. Electron tomography: A 3d view of the subcellular world. Analytical Chemistry, 79(21):7949-7957, November 2007. ISSN 0003-2700. URL http://dx.doi .org/10.1021/ac071982u. (Cited on page 3.)

[4] Marco Stampanoni, Rajmund Mokso, Federica Marone, Joan Vila-Comamala, Sergey Gorelick, Pavel Trtik, Konstantin Jefimovs, and Christian David. Phase-contrast tomography at the nanoscale using hard $\mathrm{x}$ rays. Phys. Rev. B, 81(14):140105, Apr 2010. URL http://dx.doi.org/10.1103/PhysRevB.81.140105. (Cited on page 3.)

[5] J.D. Woodward and J.N. Maina. A $3 \mathrm{D}$ digital reconstruction of the components of the gas exchange tissue of the lung of the muscovy duck, Cairina moschata. J.Anat., 206(5):477492, May 2005. URL http://dx. doi .org/10.1111/j . 1469-7580. 2005.00413.x. (Cited on pages 3, 5, and 12.)

[6] Minsky Marvin. Microscopy apparatus, December 1961. URL http://www. freepatentsonline.com/3013467.html. (Cited on page 3.)

[7] GN Hounsfield. Historical notes on computerized axial tomography. Journal of the Canadian Association of Radiologists, 27(3):135, 1976. (Cited on pages 4 and 15.)

[8] Ulrich Bonse. X-ray imaging: past and present. volume 7078, page 707802. SPIE, 2008. URL http://dx.doi.org/10.1117/12. 794694. (Cited on pages 4 and 16.)

[9] Roberto Danovaro, Antonio Dell'Anno, Antonio Pusceddu, Cristina Gambi, Iben Heiner, and Reinhardt Mobjerg Kristensen. The first metazoa living in permanently anoxic conditions. BMC Biology, 8(1):30, 2010. ISSN 1741-7007. URL http://dx.doi.org/ 10.1186/1741-7007-8-30. (Cited on page 4.)

[10] Ewald R Weibel. What makes a good lung? Swiss Med Wkly, 139(27-28):375-386, Jul 2009. (Cited on pages 4, 5, 9, 11, 62, 76, and 83.)

[11] Johannes C. Schittny and Peter H. Burri. Development and Growth of the Lung, chapter 5, pages 91-114. McGraw-Hill Professional, 2008. URL http://books.google.com/books?id= 3FjqNJI1480C\&pg=PT150. (Cited on pages 4, 9, 10, 11, 12, and 62.) 
[12] Dallas M. Hyde, Shelley A. Blozis, Mark V. Avdalovic, Lei F. Putney, Rachel Dettorre, Nathanial J. Quesenberry, Paramjit Singh, and Nancy K. Tyler. Alveoli increase in number but not size from birth to adulthood in rhesus monkeys. Am J Physiol Lung Cell Mol Physiol, 293(3):L570-579, 2007. URL http://dx.doi.org/10. 1152/ajplung.00467.2006. (Cited on pages 4 and 59.)

[13] A. Tsuda, R.A. Rogers, P.E. Hydon, and J.P. Butler. Chaotic mixing deep in the lung. Proceedings of the National Academy of Sciences of the United States of America, 99(15):10173-10178, July 2002. URL http://dx.doi.org/10.1073/pnas.102318299. (Cited on pages 4 and 6.)

[14] Eric A. Hoffman and Deokiee Chon. Computed Tomography Studies of Lung Ventilation and Perfusion. Proc Am Thorac Soc, 2(6):492-498, 2005. URL http://dx.doi.org/10.1513/pats. 200509-099DS. (Cited on page 4.)

[15] S. Bayat, L. Porra, H. Suhonen, C. Nemoz, P. Suortti, and A. R. Sovijärvi. Differences in the time course of proximal and distal airway response to inhaled histamine studied by synchrotron radiation CT. J. Appl. Physiol., 100:1964-1973, Jun 2006. URL http://dx.doi.org/10.1152/japplphysiol.00594.2005. (Cited on pages 4 and 57 .)

[16] S. Bayat, L. Porra, H. Suhonen, P. Suortti, and A. R. Sovijärvi. Paradoxical conducting airway responses and heterogeneous regional ventilation after histamine inhalation in rabbit studied by synchrotron radiation CT. J. Appl. Physiol., 106:1949-1958, Jun 2009. URL http://dx.doi.org/10.1152/japplphysiol.90550. 2008.

[17] Sonja I. Mund, Marco Stampanoni, and Johannes C. Schittny. Developmental alveolarization of the mouse lung. Developmental Dynamics, 237(8):2108-2116, 2008. URL http://dx.doi.org/10. $1002 / d v d y .21633$. (Cited on pages 36 and 62.)

[18] Johannes C. Schittny, Sonja I. Mund, and Marco Stampanoni. Evidence and structural mechanism for late lung alveolarization. Am J Physiol Lung Cell Mol Physiol, 294(2):L246-254, 2008. URL http://dx.doi.org/10.1152/ajplung.00296.2007. (Cited on pages $10,11,56,57$, and 62 .)

[19] A. Tsuda, N. Filipovic, D. Haberthür, R. Dickie, Y. Matsui, M. Stampanoni, and J. C. Schittny. Finite element $3 \mathrm{D}$ reconstruction of the pulmonary acinus imaged by synchrotron Xray tomography. J Appl Physiol, 105(3):964-976, 2008. URL http://dx.doi.org/10.1152/japplphysiol. 90546.2008. (Cited on pages $4,36,63$, and 83 .)

[20] Patrick J. Lynch. Bronchial anatomy detail of alveoli and lung circulation, 2010. URL http://en.wikipedia.org/wiki/File: Bronchial_anatomy.jpg. [Online; 20.04.2010]. (Cited on page 5.)

[21] P. H. Burri, J. Dbaly, and E. R. Weibel. The postnatal growth of the rat lung. Anat. Rec., 178:711-730, Apr 1974. (Cited on pages 5 and 12.) 
[22] Paul Verhoeven. Hollow Man. Columbia Pictures Corporation, August 2000. URL http://www. imdb. com/title/tt0164052. (Cited on page 9.)

[23] Ichiro Takahashi and Hiroshi Kiyono. Gut as the Largest Immunologic Tissue. JPEN J Parenter Enteral Nutr, 23:S7-12, 1999. URL http://dx.doi.org/10.1177/014860719902300503. (Cited on page 9.)

[24] G.B. Haycock, G.J. Schwartz, and D.H. Wisotsky. Geometric method for measuring body surface area: A height-weight formula validated in infants, children, and adults+. The Journal of Pediatrics, 93(1):62-66, 1978. URL http://dx.doi.org/10.1016/ S0022-3476 (78)80601-5. (Cited on page 9.)

[25] Lewis F. Richardson. The problem of contiguity: An appendix to Statistic of Deadly Quarrels, volume 6, pages 139-187. Ann Arbor, Mich. : The Society, 1961. (Cited on page 9.)

[26] B. Mandelbrot. How long is the coast of Britain? Statistical self-similarity and fractional dimension. Science, 156(3775):636, 1967. URL http://dx.doi.org/10.1126/science.156.3775.636. (Cited on pages 9 and 58.)

[27] J.C. Schittny and P.H. Burri. Morphogenesis of the Mammalian Lung: Aspects of Structure and Extracellular Matrix Components, pages 275-317. Marcel Dekker Inc, 2004. (Cited on pages 9 and 10.)

[28] M. Rodriguez, S. Bur, A. Favre, and E. R. Weibel. Pulmonary acinus: Geometry and morphometry of the peripheral airway system in rat and rabbit. American Journal of Anatomy, 180(2):143155, 1987. URL http://dx.doi.org/10.1002/aja.1001800204. (Cited on pages 10, 62, and 83.)

[29] P.H. Burri. Lung Disease, chapter Lung development and pulmonary angiogenesis, pages 122-151. Oxford University Press, New York., 1999. (Cited on page 10.)

[30] Beatrice Haefeli-Bleuer and Ewald R. Weibel. Morphometry of the human pulmonary acinus. The Anatomical Record, 220(4):401414, 1988. URL http://dx.doi.org/10.1002/ar.1092200410. (Cited on page 11.)

[31] Ewald R Weibel. Morphometry of the human lung. Springer-Verlag, Berlin, Göttingen, etc., 1963. (Cited on pages 11 and 42.)

[32] Patrick J. Lynch. Lungs diagram with internal detail, 2010. URL http://en.wikipedia.org/wiki/File:Lungs_ diagram_detailed.svg. [Online; 18.03.2010]. (Cited on page 12.)

[33] D. Massaro, N. Teich, S. Maxwell, G. D. Massaro, and P. Whitney. Postnatal development of alveoli. regulation and evidence for a critical period in rats. J Clin Invest, 76(4):1297-1305, Oct 1985. URL http://dx.doi.org/10.1172/JCI112103. (Cited on pages 12 and 32.)

[34] Matthias Ochs. A brief update on lung stereology. Journal of Microscopy, 222(3):188-200, 2006. URL http://dx.doi.org/10. 1111/j.1365-2818.2006.01587.x. (Cited on page 12.) 
[35] Connie C. W. Hsia, Dallas M. Hyde, Matthias Ochs, and Ewald R. Weibel. An Official Research Policy Statement of the American Thoracic Society/European Respiratory Society: Standards for Quantitative Assessment of Lung Structure. Am. J. Respir. Crit. Care Med., 181(4):394-418, 2010. URL http://dx.doi.org/10. 1164/rccm. 200809-1522ST. (Cited on pages 12, 32, and 91.)

[36] C. Hintermüller, F. Marone, A. Isenegger, and M. Stampanoni. Image processing pipeline for synchrotron-radiation-based tomographic microscopy. Journal of Synchrotron Radiation, 17(4), Jul 2010. URL http://dx. doi.org/10.1107/S0909049510011830. (Cited on pages 13, 15, 26, 63, and 64.)

[37] Graham Chapman, John Cleese, Terry Gilliam, Eric Idle, Terry Jones, and Michael Palin. The Meaning of Life. Celandine Films, March 1983. URL http://is.gd/bpLvW. (Cited on page 15.)

[38] M. Stampanoni, A. Groso, A. Isenegger, G. Mikuljan, Q. Chen, A. Bertrand, S. Henein, R. Betemps, U. Frommherz, P. Böhler, D. Meister, M. Lange, and R. Abela. Trends in synchrotron-based tomographic imaging: the SLS experience. In Society of PhotoOptical Instrumentation Engineers (SPIE) Conference Series, volume 6318 of Society of Photo-Optical Instrumentation Engineers (SPIE) Conference Series, Aug. 2006. URL http://dx.doi.org/10.1117/ 12.679497. (Cited on pages $15,25,63$, and 77.)

[39] Gabriel Frank. X-ray apparatus, May 1942. URL http://www. freepatentsonline.com/2281931. html. (Cited on page 15.)

[40] J. Hsieh. Computed tomography: Principles, design, artifacts, and recent advances. SPIE press, 2003. URL http://books.google. com/books? id=JX__LLXFHkC. (Cited on pages 15, 16, 17, and 18.)

[41] W.L. Robb. Perspective on the first 10 years of the CT scanner industry. Academic Radiology, 10(7):756-760, 2003. URL http://dx. doi. org/10.1016/S1076-6332 (03)80121-6. (Cited on page 16.)

[42] AM Cormack. Representation of a Function by Its Line Integrals, with Some Radiological Applications. Journal of Applied Physics, 34:2722, 1963. URL http://dx.doi.org/10.1063/1.1729798. (Cited on page 16.)

[43] AM Cormack. Nobel lecture, 1979. URL http: //nobelprize.org/nobel_prizes/medicine/laureates/1979/ cormack-lecture.pdf. (Cited on page 16.)

[44] EN) Hounsfield, Godfrey Newbold (Newark. Apparatus for examining objects by means of penetrating radiation, February 1976. URL http://www. freepatentsonline. com/3940625. html. (Cited on page 16.)

[45] L. Grodzins. Optimum energies for x-ray transmission tomography of small samples:: Applications of synchrotron radiation to computerized tomography I. Nuclear Instruments and Methods in Physics Research, 206(3):541-545, 1983. URL http://dx.doi.org/ 10.1016/0167-5087 (83) 90393-9. (Cited on page 16.) 
[46] L. Grodzins. Critical absorption tomography of small samples:: Proposed applications of synchrotron radiation to computerized tomography II. Nuclear Instruments and Methods in Physics Research, 206(3):547-552, 1983. URL http://dx.doi.org/10.1016/ 0167- 5087 (83) $90394-0$. (Cited on page 16.)

[47] Arthur H. Compton. A quantum theory of the scattering of x-rays by light elements. Phys. Rev., 21(5):483-502, May 1923. URL http://ds.doi.org/10.1103/PhysRev.21.483. (Cited on page 17.)

[48] Marco Stampanoni, Gunther Borchert, Peter Wyss, Rafael Abela, Bruce Patterson, Steven Hunt, Detlef Vermeulen, and Peter Ruegsegger. High resolution x-ray detector for synchrotronbased microtomography. Nuclear Instruments and Methods in Physics Research Section A: Accelerators, Spectrometers, Detectors and Associated Equipment, 491(1-2):291-301, September 2002. URL http://dx.doi.org/10.1016/S0168-9002(02)01167-1. (Cited on pages 17,44 , and 56 .)

[49] Harold Elford Johns and John Robert Cunningham. Physics of Radiology, Fourth Edition. Charles C. Thomas Publisher, 4 sub edition, 2 1983. ISBN 9780398046699. URL http://amazon.com/ o/ASIN/0398046697/. (Cited on page 18.)

[50] MJ Berger, JH Hubbell, SM Seltzer, J. Chang, JS Coursey, R. Sukumar, and DS Zucker. XCOM: photon cross sections database. NIST Standard Reference Database, 8:87-3597, 1998. URL http:// physics.nist.gov/PhysRefData/Xcom/Text/XCOM.html. (Cited on page 19.)

[51] Avinash C. Kak and Malcolm Slaney. Principles of computerized tomographic imaging. IEEE Press, 1988. URL http://www. slaney. org/pct/. (Cited on pages 19, 21, and 65.)

[52] Johann Radon. Über die Bestimmung von Funktionen durch ihre Integralwerte längs gewisser Mannigfaltigkeiten. Berichte Sächsische Akademie der Wissenschaften, 69:262-277, 1917. (Cited on page 20.)

[53] CE Shannon. Communication in the presence of noise. Proceedings of the IRE, 37(1):10-21, 1949. (Cited on page 21.)

[54] Marco Stampanoni. A novel approach towards hard X-ray submicrometer computer tomography with synchrotron radiation. $\mathrm{PhD}$ thesis, ETH Zürich, 2002. URL http://dx.doi.org/10.3929/ ethz-a-004494073. (Cited on pages 21 and 22.)

[55] Giorgio Margaritondo. Elements of Synchrotron Light: For Biology, Chemistry, and Medical Research (Physics). Oxford University Press, USA, 2002. ISBN 0198509316. (Cited on page 24.)

[56] SLS Web Site. Description, 2010. URL http://sls.web.psi.ch/. [Online; accessed 15-February-2010]. (Cited on pages 22 and 25.)

[57] Wikipedia. Dipole magnet - Wikipedia, The Free Encyclopedia, 2009. URL http://en.wikipedia.org/w/index. php?title= Dipole_magnet\&oldid=332257500. [Online; accessed 15-April2010]. (Cited on page 22.) 
[58] David Attwood. Soft X-Rays and Extreme Ultraviolet Radiation: Principles and Applications. Cambridge University Press, 2007. ISBN 9780521029971. (Cited on pages 23 and 24.)

[59] Joel Coen and Ethan Coen. The Big Lebowski. Polygram Filmed Entertainment, March 1998. URL http://www.imdb.com/title/ tt0118715/. (Cited on page 31.)

[6o] G. D. Massaro and D. Massaro. Formation of alveoli in rats: postnatal effect of prenatal dexamethasone. Am J Physiol, 263(1 Pt 1):L37-L41, Jul 1992. (Cited on page 32.)

[61] M. Stampanoni, A. Groso, A. Isenegger, G. Mikuljan, Q. Chen, D. Meister, M. Lange, R. Betemps, S. Henein, and R. Abela. TOMCAT: A beamline for TOmographic Microscopy and Coherent rAdiology experimenTs. AIP Conference Proceedings, 879(1):848851, 2007. URL http://dx.doi.org/10.1063/1.2436193. (Cited on pages $36,43,44,56,57$, and 62.)

[62] Stefan A Tschanz, Andrew N Makanya, Beat Haenni, and Peter $\mathrm{H}$ Burri. Effects of neonatal high-dose short-term glucocorticoid treatment on the lung: a morphologic and morphometric study in the rat. Pediatr Res, 53(1):72-80, Jan 2003. URL http:// dx. doi.org/10.1203/01.PDR.0000041513.93422.C8. (Cited on pages $41,43,44,46,49,54,57$, and 58.)

[63] D. Aykac, E.A. Hoffman, G. McLennan, and J.M. Reinhardt. Segmentation and analysis of the human airway tree from threedimensional X-ray CT images. IEEE transactions on medical imaging, 22(8):940-950, 2003. URL http://dx.doi.org/10.1109/TMI. 2003.815905. (Cited on pages 42 and 55.)

[64] A. Chaturvedi and Z. Lee. Three-dimensional segmentation and skeletonization to build an airway tree data structure for small animals. Physics in Medicine and Biology, 50(7):1405-1419, 2005. URL http://dx. doi.org/10.1088/0031-9155/50/7/005.

[65] I. Cheng, S. Nilufar, C. Flores-Mir, and A. Basu. Airway segmentation and measurement in CT images. In Engineering in Medicine and Biology Society, 2007. EMBS 2007. 29th Annual International Conference of the IEEE, pages 795-799, 2007.

[66] WK Chooi and SK Morcos. High resolution volume imaging of airways and lung parenchyma with multislice CT. British Journal of Radiology, 77(Special Issue 1):S98, 2004. URL http://dx. doi. org/10.1259/bj r/27596725.

[67] JR Dame Carroll, A. Chandra, AS Jones, N. Berend, JS Magnussen, and GG King. Airway dimensions measured from micro-computed tomography and high-resolution computed tomography. European Respiratory Journal, 28(4):712, 2006. URL http://dx.doi.org/10.1183/09031936.06.00012405.

[68] B. Driehuys, J. Walker, J. Pollaro, G.P. Cofer, N. Mistry, D. Schwartz, and G.A. Johnson. ${ }^{3} \mathrm{He}$ MRI in mouse models of asthma. Magnetic resonance in medicine: official journal of the Society of Magnetic Resonance in Medicine/Society of Magnetic Resonance in Medicine, 58(5):893, 2007. URL http://dx.doi.org/10.1002/ mrm. 21306. 
[69] YA Kvistedal, M. Tawhai, P. Hunter, and PMF Nielsen. High resolution $3 \mathrm{D}$ imaging of lung tissue using structured light microscopy. In Engineering in Medicine and Biology Society, 2005. IEEE-EMBS 2005. 27th Annual International Conference of the, pages 193-195. IEEE, 2006.

[70] J. Ley-Zaporozhan, S. Ley, and H.U. Kauczor. Morphological and functional imaging in COPD with CT and MRI: present and future. European Radiology, 18(3):510-521, 2008. URL http://dx. doi.org/10.1007/s00330-007-0772-1.

[71] M. Scadeng, H.B. Rossiter, D.J. Dubowitz, and E.C. Breen. Highresolution three-dimensional magnetic resonance imaging of mouse lung in situ. Investigative radiology, 42(1):50, 2007. URL http://dx.doi.org/10.1097/01.rli.0000248850.13221.61.

[72] T. Sera, H. Fujioka, H. Yokota, A. Makinouchi, R. Himeno, R.C. Schroter, and K. Tanishita. Three-dimensional visualization and morphometry of small airways from microfocal X-ray computed tomography. Journal of biomechanics, 36(11):1587-1594, 2003. URL http://dx.doi.org/10.1016/S0021-9290(03)00179-9. (Cited on page 55. .)

[73] M.H. Tawhai, P. Hunter, J. Tschirren, J. Reinhardt, G. McLennan, and E.A. Hoffman. CT-based geometry analysis and finite element models of the human and ovine bronchial tree. Journal of Applied Physiology, 97(6):2310, 2004. URL http://dx. doi .org/10. 1152/japplphysiol.00520.2004.

[74] C. van Ertbruggen, C. Hirsch, and M. Paiva. Anatomically based three-dimensional model of airways to simulate flow and particle transport using computational fluid dynamics. Journal of Applied Physiology, 98(3):970, 2005. URL http://dx.doi.org/10. 1152/japplphysiol.00795.2004. (Cited on page 42.)

[75] Peter Gehr, Marianne Bachofen, and Ewald R. Weibel. The normal human lung - ultrastructure and morphometric estimation of diffusion capacity. Respiration Physiology, 32:121-140, Feb. 1978. (Cited on page 42.)

[76] MJ Cookson, CJ Davies, A. Entwistle, and WF Whimster. The microanatomy of the alveolar duct of the human lung imaged by confocal microscpy and visualised with computerbased $3 \mathrm{D}$ reconstruction. Computerized medical imaging and graphics, 17(3):201-210, 1993. URL http://dx. doi.org/10.1016/ $0895-6111$ (93) $90044-$ N. (Cited on pages 42 and 55.)

[77] N. Berend, AC Rynell, and HE Ward. Structure of a human pulmonary acinus. British Medical Journal, 46(2):117, 1991. URL http://dx.doi.org/10.1136/thx.46.2.117. (Cited on pages 54 and 85.$)$

[78] T. Honda, H. Ota, K. Arai, M. Hayama, K. Fujimoto, Y. Yamazaki, and M. Haniuda. Three-dimensional analysis of alveolar structure in usual interstitial pneumonia. Virchows Archiv, 441(1):47-52, 2002. URL http://dx.doi.org/10.1007/ s00428- $001-0567-8$. 
[79] H.D. Litzlbauer, C. Neuhaeuser, A. Moell, S. Greschus, A. Breithecker, F.E. Franke, W. Kummer, and W.S. Rau. Threedimensional imaging and morphometric analysis of alveolar tissue from microfocal X-ray-computed tomography. American Journal of Physiology- Lung Cellular and Molecular Physiology, 291(3): L535, 2006. URL http://dx.doi.org/10.1152/ajplung.00088. 2005. (Cited on pages 55,83 , and 85 .)

[8o] RR Mercer, JM Laco, and JD Crapo. Three-dimensional reconstruction of alveoli in the rat lung for pressure-volume relationships. Journal of Applied Physiology, 62(4):1480, 1987. (Cited on page 54.)

[81] SH Randell, RR Mercer, and SL Young. Postnatal growth of pulmonary acini and alveoli in normal and oxygen-exposed rats studied by serial section reconstructions. American Journal of Anatomy, 186(1):55-68, 1989. URL http://dx.doi.org/10.1002/ aja.1001860105. (Cited on page 54.)

[82] GP Stelter, JE Hansen, and DG Fairchild. A three-dimensional reconstruction of lung parenchyma. The American review of respiratory disease, 94(1):79, 1966. (Cited on page 54.)

[83] Henrik Watz, Andreas Breithecker, Wigbert Stephan Rau, and Andres Kriete. Micro-CT of the Human Lung: Imaging of Alveoli and Virtual Endoscopy of an Alveolar Duct in a Normal Lung and in a Lung with Centrilobular Emphysema-Initial Observations. Radiology, 236(3):1053-1058, 2005. URL http: $/ / \mathrm{dx}$.doi.org/10.1148/radiol.2363041142. (Cited on pages 42 and 55.)

[84] J.C. Schittny, M. Paulsson, C. Vallan, P. H. Burri, N. Kedei, and D. Aeschlimann. Protein cross-linking mediated by tissue transglutaminase correlates with the maturation of extracellular matrices during lung development. Am J Respir Cell Mol Biol, 17(3): 334-343, Sep 1997. (Cited on page 43.)

[85] J.C. Schittny, V. Djonov, L. Fine, and P.H. Burri. Programmed cell death contributes to postnatal lung development. American Journal of Respiratory Cell and Molecular Biology, 18(6):786-793, June 1998. URL http://aj rcmb. atsjournals . org/cgi/content/ abstract/18/6/786. (Cited on page 43.)

[86] W. Scherle. A simple method for volumetry of organs in quantitative stereology. Mikroskopie, 26(1):57-60, Jun 1970. (Cited on page 44.)

[87] D.H. Ballard and C.M. Brown. Computer Vision Prentice-Hall. Englewood Cliffs, NJ, USA, 1982. (Cited on page 46.)

[88] Davies ER. Machine vision: theory, algorithms, practicalities. Academics Press, 1990.

[89] R.C. Gonzalez and E. Richard. Woods, Digital image processing. Addison Wisley, 1992. (Cited on page 46.)

[9o] R Schneiders. A grid-based algorithm for the generation of hexahedral element meshes. Engineering with Computers, 12(3-4):168177, 1996. URL http://dx.doi. org/10.1007/BF01198732. (Cited on pages 48,49 , and 60 .) 
[91] KJ Bathe. Finite element procedures. 1995, 1995. (Cited on page 49.)

[92] L.A. Freitag and P. Plassmann. Local optimization-based simplicial mesh untangling and improvement. International Journal for Numerical Methods in Engineering, 49(1):109-125, 2000. (Cited on page 49.)

[93] M. Roth-Kleiner, T.M. Berger, M.R. Tarek, P.H. Burri, and J.C. Schittny. Neonatal dexamethasone induces premature microvascular maturation of the alveolar capillary network. Developmental Dynamics, 233(4):1261-1271, 2005. URL http://dx.doi.org/10. 1002/dvdy.20447. (Cited on page 53.)

[94] E.A. Boyden. The structure of the pulmonary acinus in a child of six years and eight months. American Journal of Anatomy, 132 (3):275-299, 1971. (Cited on page 54.)

[95] B. Haefeli-Bleuer and E.R. Weibel. Morphometry of the human pulmonary acinus. The Anatomical Record, 220(4):401-414, 1988.

[96] J.P. Schreider and O.G. Raabe. Structure of the human respiratory acinus. American Journal of Anatomy, 162(3):221-232, 1981. (Cited on page 54.)

[97] JE Hansen and EP Ampaya. Human air space shapes, sizes, areas, and volumes. Journal of Applied Physiology, 38(6):990, 1975. (Cited on page 54.)

[98] JE Hansen, EP Ampaya, GH Bryant, and JJ Navin. Branching pattern of airways and air spaces of a single human terminal bronchiole. Journal of Applied Physiology, 38(6):983, 1975.

[99] H. Parker, K. Horsfield, and G. Cumming. Morphology of distal airways in the human lung. J Appl Physiol, 31(3):386-391, 1971. (Cited on page 54.)

[10o] EA Hoffman and WB Gefter. Multimodality imaging of the upper airway: MRI, MR spectroscopy, and ultrafast X-ray CT. Progress in clinical and biological research, 345:291, 1990. (Cited on page 55.)

[101] G. Sundaramoorthy, JD Hoford, EA Hoffman, and WE Higgins. Impromptu: a system for automatic $3 \mathrm{D}$ medical imageanalysis. Computerized medical imaging and graphics, 19(1):131-143, 1995. URL http://dx. doi . org/10.1016/0895-6111(94) 00042-5. (Cited on page 55.)

[102] N. Hanna, D. Saltzman, D. Mukai, Z. Chen, S. Sasse, J. Milliken, S. Guo, W. Jung, H. Colt, and M. Brenner. Two-dimensional and 3-dimensional optical coherence tomographic imaging of the airway, lung, and pleura. The Journal of Thoracic and Cardiovascular Surgery, 129(3):615-622, 2005. URL http://dx.doi.org/10. 1016/j . jtcvs . 2004 .10.022. (Cited on page 55.)

[103] E.J.R. van Beek and E.A. Hoffman. Functional imaging: CT and MRI. Clinics in chest medicine, 29(1):195-216, 2008. URL http: //dx.doi.org/10.1016/j .ccm.2007.12.003. (Cited on page 55.) 
[104] U. Bonse and F. Busch. X-ray computed microtomography ( $\mu \mathrm{CT})$ using synchrotron radiation (SR). Progress in biophysics and molecular biology, 65(1-2):133-169, 1996. (Cited on pages 55 and 57.)

[105] A.M. Pena, A. Fabre, D. Debarre, J. Marchal-Somme, B. Crestani, J.L. Martin, E. Beaurepaire, and M.C. Schanne-Klein. Threedimensional investigation and scoring of extracellular matrix remodeling during lung fibrosis using multiphoton microscopy. Microscopy Research and Technique, 70(2):162, 2007. URL http: //dx.doi.org/10.1002/jemt.20400. (Cited on page 55.)

[106] WF Whimster. The microanatomy of the alveolar duct system. British Medical Journal, 25(2):141, 1970. URL http://dx.doi.org/ 10.1136/thx.25.2.141. (Cited on page 55.)

[107] RH Brown, CJ Herold, CA Hirshman, EA Zerhouni, and W. Mitzner. In vivo measurements of airway reactivity using high-resolution computed tomography. The American review of respiratory disease, 144(1):208, 1991. (Cited on page 55.)

[108] AE McNamara, NL Muller, M. Okazawa, J. Arntorp, BR Wiggs, and PD Pare. Airway narrowing in excised canine lungs measured by high-resolution computed tomography. Journal of Applied Physiology, 73(1):307, 1992. (Cited on page 55.)

[109] WE Higgins, K. Ramaswamy, RD Swift, G. McLennan, and EA Hoffman. Virtual bronchoscopy for three-dimensional pulmonary image assessment: state of the art and future needs. $R a-$ diographics, 18(3):761, 1998. (Cited on page 55.)

[110] W. Park, E.A. Hoffman, and M. Sonka. Segmentation of intrathoracic airway trees: a fuzzy logic approach. IEEE Transactions on Medical Imaging, 17(4):489-497, 1998. URL http://dx.doi.org/ $10.1109 / 42.730394$.

[111] JM Reinhardt, N. D'Souza, and EA Hoffman. Accurate measurement of intrathoracic airways. IEEE transactions on medical imaging, 16(6):820-827, 1997. URL http://dx.doi.org/10.1109/ 42.650878 .

[112] V. Sauret, PM Halson, IW Brown, JS Fleming, and AG Bailey. Study of the three-dimensional geometry of the central conducting airways in man using computed tomographic (CT) images. Journal of Anatomy, 200(2):123, 2002. URL http://dx.doi.org/ 10.1046/j.0021-8782.2001.00018.x.

[113] SA Wood, JD Hoford, EA Hoffman, E. Zerhouni, and W. Mitzner. A method for measurement of cross sectional area, segment length, and branching angle of airway tree structures in situ. Computerized medical imaging and graphics, 19(1):145-152, 1995. URL http://dx. doi .org/10.1016/0895 - 6111(94)00034 - 4 .

[114] SA Wood, EA Zerhouni, JD Hoford, EA Hoffman, and W. Mitzner. Measurement of three-dimensional lung tree structures by using computed tomography. Journal of Applied Physiology, 79(5):1687, 1995. (Cited on page 55.) 
[115] A. C. Langheinrich, R. M. Bohle, A. Breithecker, D. Lommel, and W. S. Rau. Micro-computed tomography of the vasculature in parenchymal organs and lung alveoli. Rofo, 176(9):1219-1225, Sep 2004. URL http://dx.doi.org/10.1055/s-2004-813403. (Cited on pages 55 and 83.)

[116] Atsuo IIda. X-ray analysis by synchrotron radiation-X-ray fluorescence analysis and XAFS. Biomed Res, 14(3):188-195, 2003. (Cited on page 56.)

[117] S.M. Jorgensen, O. Demirkaya, and E.L. Ritman. Threedimensional imaging of vasculature and parenchyma in intact rodent organs with X-ray micro-CT. American Journal of PhysiologyHeart and Circulatory Physiology, 275(3):H1103, 1998. (Cited on page 57.)

[118] S. Jheon, H.S. Youn, H.T. Kim, G.H. Choi, and J.K. Kim. Highresolution X-ray refraction imaging of rat lung and histological correlations. Microscopy research and technique, 69(8):656-659, 2006. URL http://dx.doi.org/10.1002/jemt.20335. (Cited on page 57.)

[119] S. Monfraix, S. Bayat, L. Porra, G. Berruyer, C. Nemoz, W. Thomlinson, P. Suortti, and A.R.A. Sovijärvi. Quantitative measurement of regional lung gas volume by synchrotron radiation computed tomography. Physics in medicine $\mathcal{E}$ biology(Print), 50(1): 1-11, 2005. URL http://dx.doi.org/10.1088/0031-9155/50/1/ 001.

[120] T. Sera, K. Uesugi, R. Himeno, and N. Yagi. Small airway changes in healthy and ovalbumin-treated mice during quasistatic lung inflation. Respiratory Physiology \& Neurobiology, 156(3): 304-311, 2007. URL http://dx.doi.org/10.1016/j. resp. 2006. 11.006 .

[121] T. Sera, K. Uesugi, and N. Yagi. Morphometric deformations of small airways and alveoli under quasi-static inflation process. Journal of PHYSIOLOGICAL ANTHROPOLOGY and Applied Human Science, 24(4):465-468, 2005. URL http://dx.doi.org/10. 2114/jpa.24.465. (Cited on page 57.)

[122] H. Ikura, K. Shimizu, J. Ikezoe, T. Nagareda, and N. Yagi. In vitro evaluation of normal and abnormal lungs with ultra-highresolution CT. Journal of Thoracic Imaging, 19(1):8, 2004. (Cited on page 57. .)

[123] Stefan A Tschanz and Peter H Burri. A new approach to detect structural differences in lung parenchyma using digital image analysis. Experimental Lung Research, 28(6):457-471, September 2002. URL http://dx. doi. org/10. 1080/01902140290096719. (Cited on page 63.)

[124] Cedric Luyet, Peter H Burri, and Johannes C Schittny. Suppression of cell proliferation and programmed cell death by dexamethasone during postnatal lung development. American Journal of Physiology-Lung Cellular and Molecular Physiology, 282(3):477-483, 2002. URL http://dx.doi.org/10.1152/ajplung.00406.2000. (Cited on page 63.) 
[125] LA Shepp and BF Logan. The Fourier reconstruction of a head section. IEEE Trans. Nucl. Sci, 21(1):21-43, 1974. (Cited on page 66.)

[126] B. A. Dowd, G. H. Campbell, R. B. Marr, V. V. Nagarkar, S. V. Tipnis, L. Axe, and D. P. Siddons. Developments in synchrotron x-ray computed microtomography at the National Synchrotron Light Source. SPIE Developments in X-Ray Tomography II, Denver, CO (US), o7/18/1999-07/23/1999, 3772:224-236, September 1999. URL http://dx.doi.org/10.1117/12.363725. (Cited on page 69.)

[127] Federica Marone, Christoph Hintermüller, Roman Geus, and Marco Stampanoni. Towards real-time tomography: Fast reconstruction algorithms and GPU implementation. In IEEE Nuclear Science Symposium Conference Record, 2008. NSS'08, pages 555561. IEEE, 2008. (Cited on page 69.)

[128] Nobuyuki Otsu. A threshold selection method from gray-level histograms. IEEE Transactions on Systems, Man, and Cybernetics, 9 (1):62-66, 1979. (Cited on pages 69 and 74.)

[129] S.W. Zucker. Region growing: Childhood and adolescence. Computer graphics and image processing, 5(3):382-399, 1976. (Cited on pages 72 and 87 .)

[130] P.E. Danielsson. Euclidean distance mapping. Computer Graphics and image processing, 14(227-248):6-8, 1980. (Cited on page 74.)

[131] Hans Nathan Kohn. Zur Histologie der indurirenden fibrinösen Pneumonie. Münchener Medicinische Wochenschrift, 40:42-45, 1893. (Cited on page 75.)

[132] F. Van Meir. The alveolar pores of Kohn in young postnatal rat lungs and their relation with type II pneumocytes. Histology and histopathology, 6(1):55, 1991. (Cited on page 75.)

[133] Wikipedia. Invader Zim - Wikipedia, The Free Encyclopedia, 2010. URL http://en.wikipedia.org/w/index.php?title= Invader_Zim\&oldid=359199292. [Online; accessed 29-April2010]. (Cited on page 83.)

[134] Maryann E. Martone, Amarnath Gupta, Mona Wong, Xufei Qian, Gina Sosinsky, Bertram Ludäscher, and Mark H. Ellisman. A cell-centered database for electron tomographic data. Journal of Structural Biology, 138(1-2):145 - 155, 2002. ISSN 1047-8477. URL http://dx.doi.org/10.1016/S1047-8477(02)00006-0. (Cited on page 83.)

[135] Vladan Lučić, Friedrich Förster, and Wolfgang Baumeister. Structural Studies by Electron Tomography: From Cells to Molecules. Annual Review of Biochemistry, 74(1):833-865, 2005. URL http://dx. doi . org/10.1146/annurev . biochem.73.011303. 074112. (Cited on page 83.)

[136] A.C. Langheinrich, B. Leithäuser, S. Greschus, S. von Gerlach, A. Breithecker, F.R. Matthias, W.S. Rau, and R.M. Bohle. Acute rat lung injury: feasibility of assessment with micro-CT. Radiology, 233(1):165, 2004. URL http://dx. doi.org/10.1148/radiol. 2331031340. (Cited on page 83.) 
[137] M. Reza Akhavan Sharif, Wilfred W. Lam, Alexei V. Ouriadov, David W. Holdsworth, and Giles E. Santyr. Comparison of hyperpolarized ${ }^{3} \mathrm{He}$ MRI rat lung volume measurement with microcomputed tomography. NMR in Biomedicine, 23(4):359-367, 2010. URL http://dx. doi.org/10.1002/nbm. 1470. (Cited on page 83.)

[138] Erik L. Ritman. Micro-Computed Tomography of the Lungs and Pulmonary-Vascular System. Proc Am Thorac Soc, 2(6):477-48o, 2005. URL http://dx.doi.org/10.1513/pats.200508-080DS. (Cited on page 83.)

[139] David Haberthür, Christoph Hintermüller, Federica Marone, Johannes C. Schittny, and Marco Stampanoni. Radiation dose optimized lateral expansion of the field of view in synchrotron radiation x-ray tomographic microscopy. Journal of Synchrotron Radiation, 2010. in Press. (Cited on page 83.)

[140] Christian Mühlfeld, Barbara Rothen-Rutishauser, Fabian Blank, Dimitri Vanhecke, Matthias Ochs, and Peter Gehr. Interactions of nanoparticles with pulmonary structures and cellular responses. Am J Physiol Lung Cell Mol Physiol, 294(5):L817-829, 2008. URL http://dx.doi.org/10.1152/ajplung.00442.2007. (Cited on page 84.)

[141] Wikipedia. Yaw, pitch, and roll - Wikipedia, The Free Encyclopedia, 2010. URL http://en.wikipedia.org/w/index.php? title=Yaw, _pitch, , and_roll\&oldid=354392227. [Online; accessed 3-May-2010]. (Cited on page 86.)

[142] Sebastien Barré. Multimodal imaging of lung tissue. Master's thesis, Biomedical Engineering, University of Bern, September 2009. (Cited on pages 85 and 86.)

[143] Josué Sznitman, Fabian Heimsch, Thomas Heimsch, Daniel Rusch, and Thomas Rösgen. Three-Dimensional Convective Alveolar Flow Induced by Rhythmic Breathing Motion of the Pulmonary Acinus. Journal of Biomechanical Engineering, 129(5):658665, 2007. URL http://dx.doi.org/10.1115/1.2768109. (Cited on page 87. .)

[144] Josué Sznitman, Thomas Heimsch, Johannes H. Wildhaber, Akira Tsuda, and Thomas Rösgen. Respiratory flow phenomena and gravitational deposition in a three-dimensional space-filling model of the pulmonary acinar tree. Journal of Biomechanical Engineering, 131(3):031010, 2009. URL http://dx.doi.org/10.1115/ 1.3049481. (Cited on page 87.)

[145] Nenad Filipovic, David Haberthür, Frank S. Henry, Danko Milasinovic, Dalibor Nikolic, Johannes C. Schittny, and Akira Tsuda. Recirculation Identified In A $3 \mathrm{D}$ Alveolar Duct Reconstructed Using Synchrotron Radiation Based X-ray Tomographic Microscopy. Am. J. Respir. Crit. Care Med., 181(1-MeetingAbstracts):A2192-, 2010. URL http://ajrccm.atsjournals.org/cgi/reprint/181/1 MeetingAbstracts/A2192.pdf. (Cited on page 87.)

[146] Wikipedia. Region growing - Wikipedia, The Free Encyclopedia, 2008. URL http://en.wikipedia.org/w/index.php?title= 
Region_growing\&oldid=260474814. [Online; accessed 11-March2009]. (Cited on page 87.)

[147] Ingmar Bitter, Robert Van Uitert, Ivo Wolf, Luis Ibáñez, and JanMartin Kuhnigk. Comparison of Four Freely Available Frameworks for Image Processing and Visualization That Use ITK. IEEE Trans Vis Comput Graph, 13(3):483-493, 2007. URL http: //dx.doi.org/10.1109/TVCG.2007.1001. (Cited on page 87.)

[148] H. A. Blum. Models for the Perception of Speech and Visual Form, chapter Transformation for Extracting New Descriptors of Shapes, page 362-380. MIT Press, Cambridge, MA, 1967. (Cited on page 88.)

[149] D. Selle, B. Preim, A. Schenk, and H.-O. Peitgen. Analysis of vasculature for liver surgical planning. Medical Imaging, IEEE Transactions on, 21(11):1344-1357, Nov. 2002. ISSN 0278-0062. URL http://dx.doi.org/10.1109/TMI.2002.801166. (Cited on page 88.)

[150] Tobias Boskamp, Daniel Rinck, Florian Link, Bernd Kummerlen, Georg Stamm, and Peter Mildenberger. New Vessel Analysis Tool for Morphometric Quantification and Visualization of Vessels in CT and MR Imaging Data Sets. Radiographics, 24(1): 287-297, 2004. URL http://dx.doi.org/10.1148/rg. 241035073. (Cited on page 88.)

[151] Dimitri Vanhecke, Daniel Studer, and Matthias Ochs. Stereology meets electron tomography: Towards quantitative $3 \mathrm{~d}$ electron microscopy. Journal of Structural Biology, In Press, Corrected Proof:-, 2007. URL http://dx.doi.org/10.1016/j.jsb. 2007.05.003. (Cited on page 91.)

[152] Stefan A Tschanz and Ewald R Weibel Peter H Burri. A simple tool for stereological assessment of digital images: the STEPanizer. in preparation, 2010. (Cited on page 91.)

[153] D. M. Hyde, N. K. Tyler, L. F. Putney, P. Singh, and H. J G Gundersen. Total number and mean size of alveoli in mammalian lung estimated using fractionator sampling and unbiased estimates of the euler characteristic of alveolar openings. Anat Rec A Discov Mol Cell Evol Biol, 277(1):216-226, Mar 2004. URL http://dx.doi.org/10.1002/ar.a.20012. (Cited on page 91.)

[154] Matthias Ochs, Jens R Nyengaard, Anja Jung, Lars Knudsen, Marion Voigt, Thorsten Wahlers, Joachim Richter, and Hans Jørgen G Gundersen. The number of alveoli in the human lung. Am J Respir Crit Care Med, 169(1):120-124, Jan 2004. URL http://dx. doi. org/10.1164/rccm.200308-11070C. (Cited on page 91.)

[155] D. C. Sterio. The unbiased estimation of number and sizes of arbitrary particles using the disector. J Microsc, $134(\mathrm{Pt} 2): 127-$ 136, May 1984. (Cited on page 91.)

[156] Douglas Adams. So Long, and Thanks for All the Fish (The Hitch Hiker's Guide to the Galaxy IV). Pan Books, 1984. ISBN o-330-28700-1. URL http://en.wikipedia.org/w/index.php? title=So_Long, _and_Thanks_for_All__the_Fish\&direction= next\&oldid=351175356. (Cited on page 93.) 
[157] Robert Bringhurst. The Elements of Typographic Style. Version 2.5. Hartley \& Marks, Publishers, Point Roberts, WA, USA, 2002. URL http://en.wikipedia.org/w/index.php?title=The Elements_of_Typographic_Style\&oldid=349204139. (Cited on page 119.) 

Last name, first name: Haberthür, David

Matriculation number: 96-103-353

I hereby declare that this thesis represents my original work and that I have used no other sources except as noted by citations.

All data, tables, figures and text citations which have been reproduced from any other source, including the Internet, have been explicitly acknowledged as such.

I am aware that in case of non-compliance, the Senate is entitled to divest me of the doctorate degree awarded to me on the basis of the present thesis, in accordance with the "Statut der Universität Bern (Universitätsstatut; UniSt)", Art. 20, of 17 December 1997.

Bern, July 14, 2010

David Haberthür 

This thesis was typeset from SVN-Revision 67 of its repository with $\mathrm{LT}_{\mathrm{E}} \mathrm{X}{ }_{\varepsilon}$ using Hermann Zapf's Palatino and Euler type faces (Type 1 PostScript fonts URW Palladio L and FPL were used). The listings are typeset in Bera Mono, originally developed by Bitstream, Inc. as "Bitstream Vera". (Type 1 PostScript fonts were made available by Malte Rosenau and Ulrich Dirr.)

The typographic style was inspired by Bringhurst's genius as presented in The Elements of Typographic Style [157]. The LATEX package classicthesis has been made by André Miede. The colors of the package have been adapted for the corporate design of the University of Bern.

NOTE: The custom size of the text block was calculated using the directions given by Mr. Bringhurst (pages 26-29 and 175/176). $10 \mathrm{pt}$ Palatino needs $133.21 \mathrm{pt}$ for the string "abcdefghijklmnopqrstuvwxyz". This yields a good line length between 24-26 pc (288-312 pt). Using a "double square text block" with a 1:2 ratio this results in a text block of 312:624 pt (which includes the headline in this design). A good alternative would be the "golden section text block" with a ratio of 1:1.62, here 312:505.44 pt. For comparison, DIV9 of the typearea package results in a line length of $389 \mathrm{pt}(32.4 \mathrm{pc})$, which is by far too long. However, this information will only be of interest for hardcore pseudotypographers like me.

Final Version as of July 14, 2010 at 10:48. 